\title{
Saline-Water Resources of North Dakota
}

GEOLOGICAL SURVEY WATER-SUPPLY PAPER $142 \mathrm{~s}$
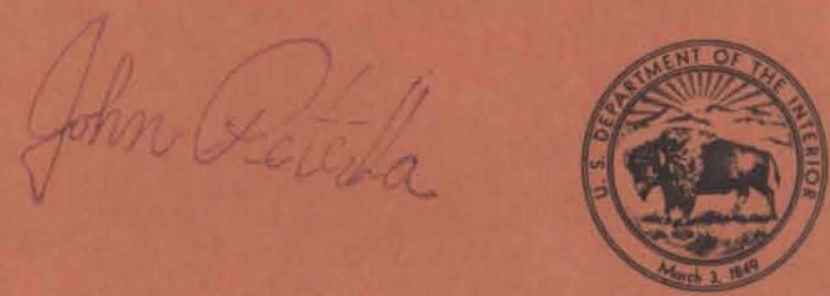


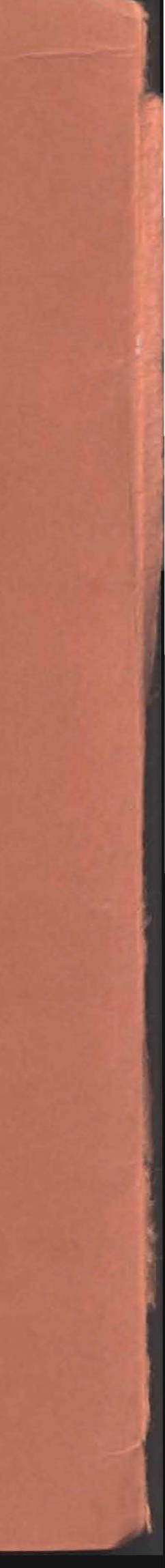




\section{Saline-Water Resources of North Dakota}

By C. J. ROBINOVE, R. H. LANGFORD, and J. W. BROOKHART

GEOLOGICAL SURVEY WATER-SUPPLY PAPER 1428

A description of the principal salinewater aquifers and surface-water bodies, with available analyses

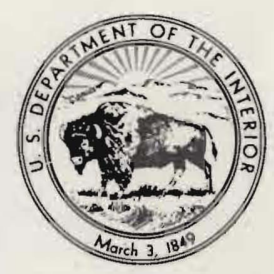




\section{UNITED STATES DEPARTMENT OF THE INTERIOR \\ FRED A. SEATON, Secretary}

\section{GEOLOGICAL SURVEY}

Thomas B. Nolan, Director 


\section{CONTENTS}

Page

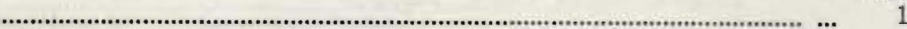

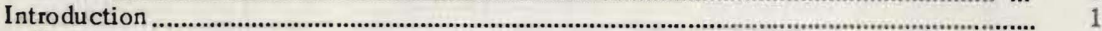

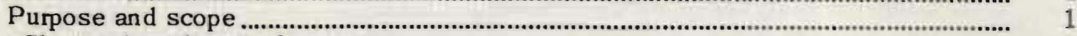

Chemical analyses of water ................................................................................................... 2

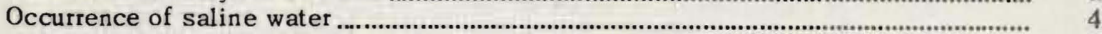

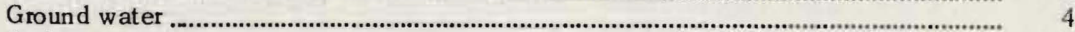

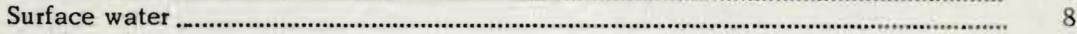

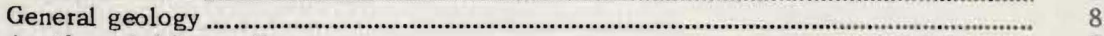

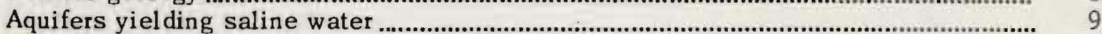

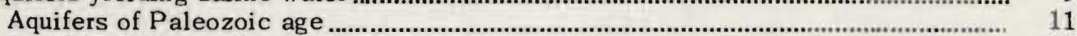

Aquifers ot Mesozoic and Cenozoic age .................................................................... 15

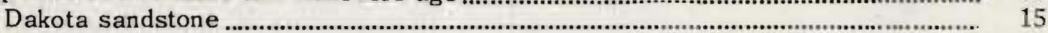

Fox Hills sandstone .................................................................................................... 24

Hell Creek formation and Fort Union formation, undifferentiated .............................. 25

Glacial drift and alluvium ............................................................................................... 38

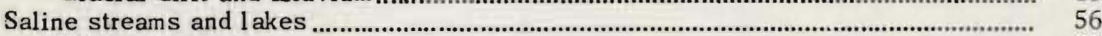

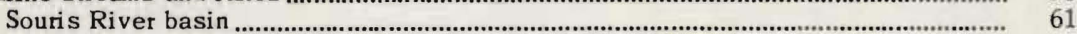

Little Missouri River basin ....................................................................................... 61

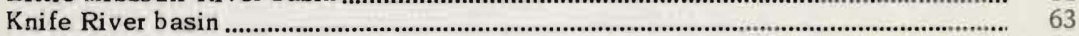

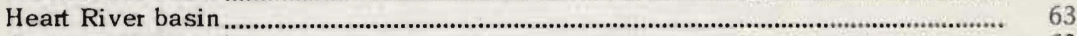

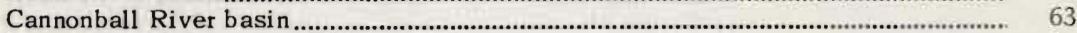

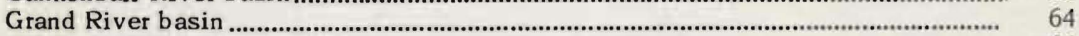

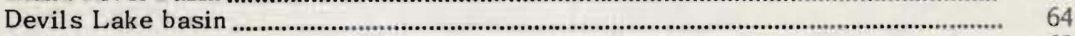

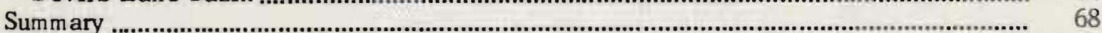

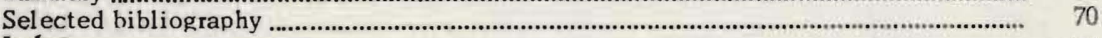

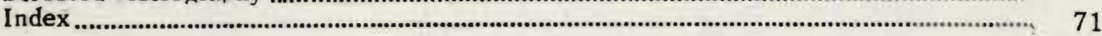

\section{ILLUSTRATIONS}

Plate 1. Map of North Dakota showing physiographic regions ............................. In pocket

Figure 1. Sketch illustrating well-numbering system ....................................................

2. Map of North Dakota showing distribution of saline-water analyses ........... 6

3. Generalized stratigraphic section of North Dakota showing saline-water aquifers

4. Chemical characteristics of saline water from consolidated rocks

5. Chemical characteristics of saline water from glacial drift and alluvium..

6. Map of North Dakota showing structure contours on the top of the Dakota sandstone and extent of geologic systems

7. Salinity-duration curves for Little Missouri, Heart, and North Fork Grand Rivers

8. Relation between water discharge and specific conduct ance, Knife River at Hazen, May 1946-September 1951

\section{TABLES}

Table 1. Description of aquifers of which some parts are capable of yielding

$50 \mathrm{gpm}$ or more of saline water to individual wells 
Table 2. Chemical analyses of saline ground water from formations of Paleozoic age.

3. Chemical analyses of saline ground water from the Dakota sandstone.

4. Chemical analyses of saline ground water from the Fox Hills sandstone...

5. Chemical analyses of saline ground water from the Hell Creek formation, Can nonball member of the Fort Union formation, and upper part of Fort Union formation, undifferentiated.

6. Chemical analyses of saline ground water from glacial drift and alluvium .... 40

7. Chemical analyses of saline streams ................................................................ 56

8. Maximum and minimum observed concentrations of mineral constituents and other characteristics, Heart River

9. Chemical analyses of saline lakes in the Devils Lake area 66 


\title{
SALINE-WATER RESOURCES OF NORTH DAKOTA
}

\author{
By C. J. Robinove, R. H. Langford, and J. W. Brookhart
}

\begin{abstract}
Natural waters in North Dakota have been arbitrarily defined in this report as saline or fresh; water containing more than 1,000 ppm (parts per million) of dissolved solids or having a specific conductance greater than 1,400 micromhos per centimeter at $25^{\circ} \mathrm{C}$ is considered to be saline. A large proportion of the available water supply of North Dakota is saline.
\end{abstract}

Saline ground water is available in small to large amounts from aquifers of Paleozoic age; in moderate to large amounts from the Dakota sandstone, in small amounts from the Fox Hills sandstone, and in small to moderate amounts from the Hell Creel formation, all of Mesozoic age; and in small to moderate amounts from the Fort Union formation of Cenozoic age; and in extremely variable amounts from the glacial drift and alluvium of Cenozoic age. In this report, yields of less than $150 \mathrm{gpm}$ (gallons per minute) to individual wells are considered to be small; 150 to $350 \mathrm{gpm}$, moderate; and more than 350 gpm, large.

Generally, the saline ground water is only slightly saline (contains 1,000 to 3,000 ppm of dissolved solids). However, some water from the Dakota sandstone is moderately saline $(3,000$ to $10,000 \mathrm{ppm})$, some from glacial drift and alluvium is very saline $(10,000$ to $35,000 \mathrm{ppm}$ ), and some from aquifers of Paleozoic age is briny (more than $35,000 \mathrm{ppm}$ ).

Saline water from aquifers of Paleozoic age is generally of the sodium chloride type; from the Dakota sandstone, of the sodium chloride sulfate type; from the Fox Hills sandstone, of the sodium bicarbonate type; from the Hell Creek formation and upper part of the Fort Union formation, of the sodium bicarbonate sulfate type; and from the Cannonball member of the Fort Union formation, of the sodium chloride type. Saline water from glacial drift and alluvium varies widely in type.

Tributaries of the Missouri River are slightly saline at low flow. Investigations of the chemical quality of the major tributaries show that the water is generally of the sodium sulfate bicarbonate type. Many lakes in the Devils Lake basin cre saline. The water in these lakes is of the sodium sulfate type and is from slightly saline in Sixmile Bay of Devils Lake to briny in eastern Stump Lake and East Devils Lake.

\section{INTRODUCTION}

PURPOSE AND SCOPE

Public Law 448 (U. S. Congress, 1952) authorized the establishment of the Saline Water Conversion Program of the U. S. Department of the Interior. The law provides for research into 
and development of practicable and economical means of producing fresh water from sea water or other saline water for the purpose of conserving and increasing the water resources of the Nation. A necessary part of the program is the determination of the quantity and quality of water that is available for demineralization.

A large proportion of the available water supply of North Dakota is saline. Water-resources investigations in North Dakota in the past have been confined principally to a search for water of the best quality available. This report is a compilation and an evaluation of the available data on the quality and quantity of saline water in North Dakota. It is one of a series of reports describing the saline-water resources in different areas of the United States.

The scope of this reconnaissance report is limited by lack of information on the quantity of saline ground water. The lack of information is due partly to the practice of abandoning wells as soon as the water is found to be too highly mineralized for general use. Therefore, only rough estimates of the yields from some water-bearing formations have been made for the evaluation of saline ground-water supplies.

The scope of this report is limited also because a complete inventory of the available analyses of saline water was not made. Analyses made by oil companies and railroad companies and by Federal agencies other than the Geological Survey are not included.

Many of the data on the saline waters of North Dakota were extracted from reports of the North Dakota Geological Survey. Some data were obtained from the North Dakota State Health Department.

This cooperative investigation was under the general supervision of A. N. Sayre, Chief, Ground Water Branch, and S. K. Love, Chief, Quality of Water Branch, of the Water Resources Division, U. S. Geological Survey.

\section{CHEMICAL ANALYSES OF WA TER}

In some areas of the United States where water generally contains less than $100 \mathrm{ppm}$ of dissolved solids, water containing $500 \mathrm{ppm}$ might be considered saline. In North Dakota, however, relatively highly mineralized water is common.

In this report natural waters are arbitrarily classified as fresh or saline on the basis of their dissolved-solids content. 
Because many of the available quality-of-water data do not show dissolved-solids concentrations, specific conductance was often used for classification. Fresh water is classified as that containing dissolved solids of less than $1,000 \mathrm{ppm}$ or having a specific conductance of less than 1,400 micromhos at $25^{\circ} \mathrm{C}$. Saline water is classified as follows:

\section{Class}

Slightly saline Moderately saline Very saline Briny.

\section{Dissolved solids (ppm)}

1,000 to 3,000

3,000 to 10,000

10,000 to 35,000

More than 35,000

\author{
Specific \\ conductance \\ (micromhos at \\ $25^{\circ} \mathrm{C}$ ) \\ 1,400 to 4,000 \\ 4,000 to 14,000 \\ 14,000 to 50,000 \\ More than 50,000
}

Tables of analyses of ground water include data from several sources (Abbott and Voedisch, 1938; Akin, 1952; Aronow, Dennis, and Akin, 1953; Dennis, 1947a, 1947b, 1947c, 1948a, and 1948b; Dennis and Akin, 1950; Dingman and Gordon, 1955; Filaseta, 1946; LaRocque, Swenson, and Greenman; ${ }^{1}$ N. Dak. Geol. Survey, 194955; Simpson, 1929; and Tychsen, 1950). Therefore, the concentrations of any given constituent are not shown to the same number of significant figures in all analyses. Percent sodium, total hardness, and noncarbonate hardness were calculated if data were sufficient. The data from Abbott and Voedisch do not show the date of collection; however, these analyses probably represent samples collected during 1936-37.

Tables of analyses of surface waters show data for samples collected and analyzed by the U. S. Geological Survey during 1946-55.

Concentrations of the constituents are given in parts per million. One part per million is a unit weight of a constituent in a million unit weights of water.

Specific conductance, which is expressed in micromhos per centimeter at $25^{\circ} \mathrm{C}$, is a measure of the ability of a solution to conduct an electrical current. It is approximately proportional to the content of dissolved solids. Most of the concentrations of dissolved solids shown in the tables of analyses represent the residue on evaporation at $180^{\circ} \mathrm{C}$. They may include organic matter and water of hydration, and they are generally greater than the sum of the determined constituents. The sum is computed by adding the concentrations in parts per million of all the

1 La Rocque, G. A. , Jr. , Swenson, H. A., and Greenman, D. W., Geology and ground-water resources of a part of the Souris River basin in North Dakota: U. S. Geol. Survey Ms. rept. 
determined constituents except bicarbonate, which is divided by 2.03 before summation.

The ratio of sodium to the principal cations (calcium, magnesium, sodium, and potassium), expressed as a percentage, is referred to as "percent sodium"; all concentrations in the computation are expressed in equivalents per million. The following table shows factors for converting parts per million to equivalents per million.

\begin{tabular}{|c|c|c|c|}
\hline Cation & Factor & Anion & Factor \\
\hline Calcium & 0.0499 & Bicarbonate......... & 0.0164 \\
\hline 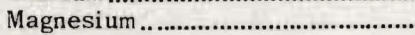 & .0822 & 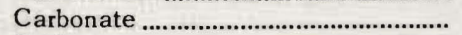 & .0333 \\
\hline Sodium & .0435 & 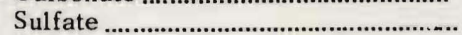 & .0208 \\
\hline 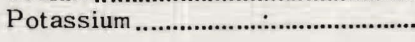 & .0256 & 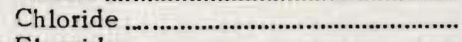 & .0282 \\
\hline & & $\begin{array}{l}\text { Fluoride } \\
\text { Nitrate }\end{array}$ & $\begin{array}{l}.0526 \\
. .0161\end{array}$ \\
\hline
\end{tabular}

A discussion of the significance of the dissolved constituents of water is not included in this report but may be found in many reports, including the current series of U. S. Geological Survey water-supply papers entitled "Quality of surface waters of the United States."

The locations of wells from which samples were collected are shown by means of numbers; the first number indicates the township, the second indicates the range, and the third indicates the section. (See fig. 1.) The lowercase letters that follow the section number indicate the location of the well within the section; the first letter indicates the quarter section, the second indicates the quarter-quarter section, and the third indicates the quarterquarter-quarter section, or 10 -acre tract. The letters are assigned in a counterclockwise direction and begin with "a" in the northeast quarter of the section or smaller subdivision. If more than one well is within the 10 -acre tract, the well number for each is assigned an additional numeral.

Plate 1 is a base map of North Dakota which may be used to locate the wells or springs. Figure 2 is a map of North Dakota showing the number of analyses of saline ground water from each county and the sampling points on saline streams and lakes.

\section{OCCURRENCE OF SALINE WATER}

\section{GROUND WATER}

Ground water may become saline by six main processes: (1) being trapped with sediments at the time of deposition in sea 

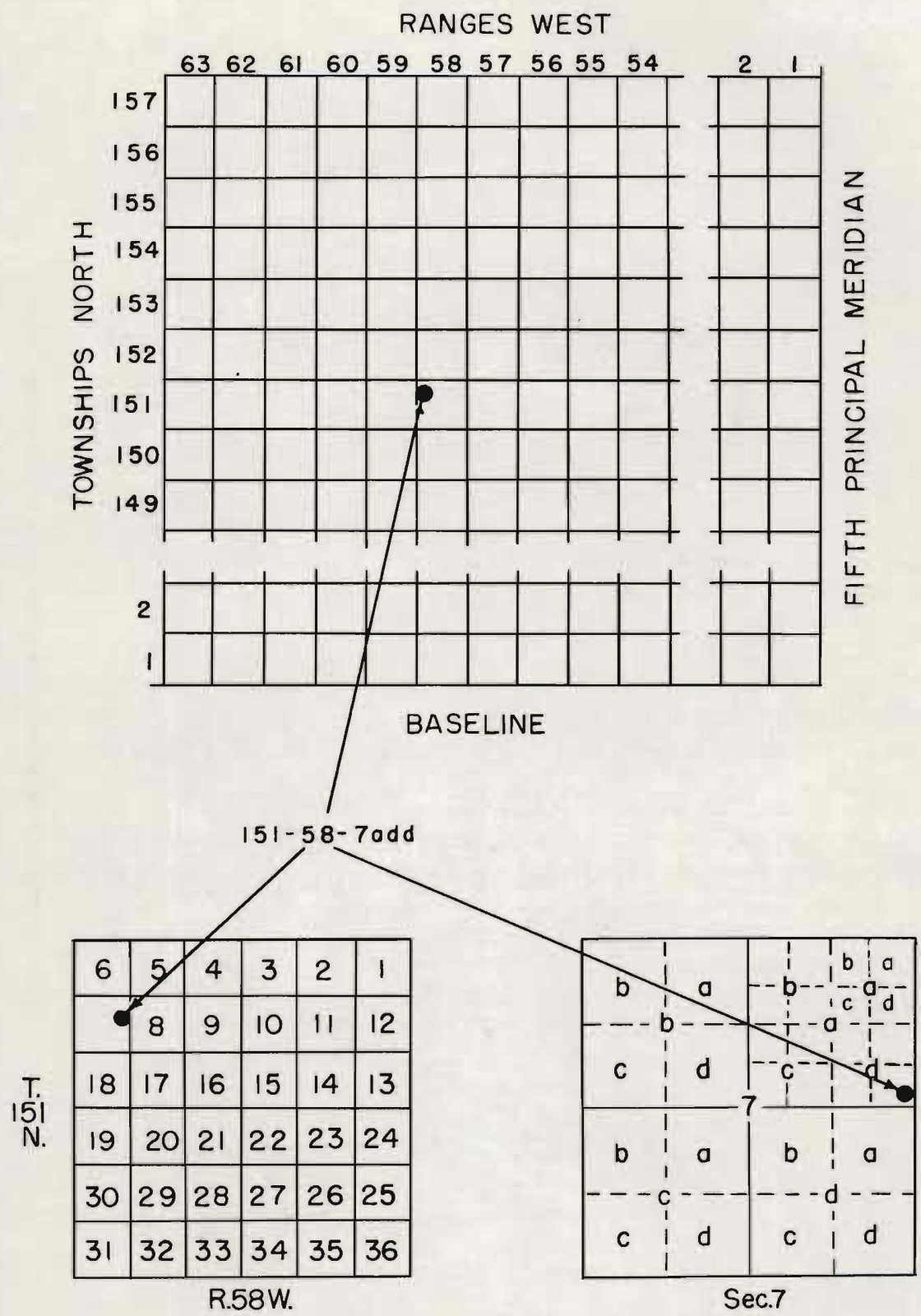

Figure 1. - Sketch illustrating well-numbering system. 


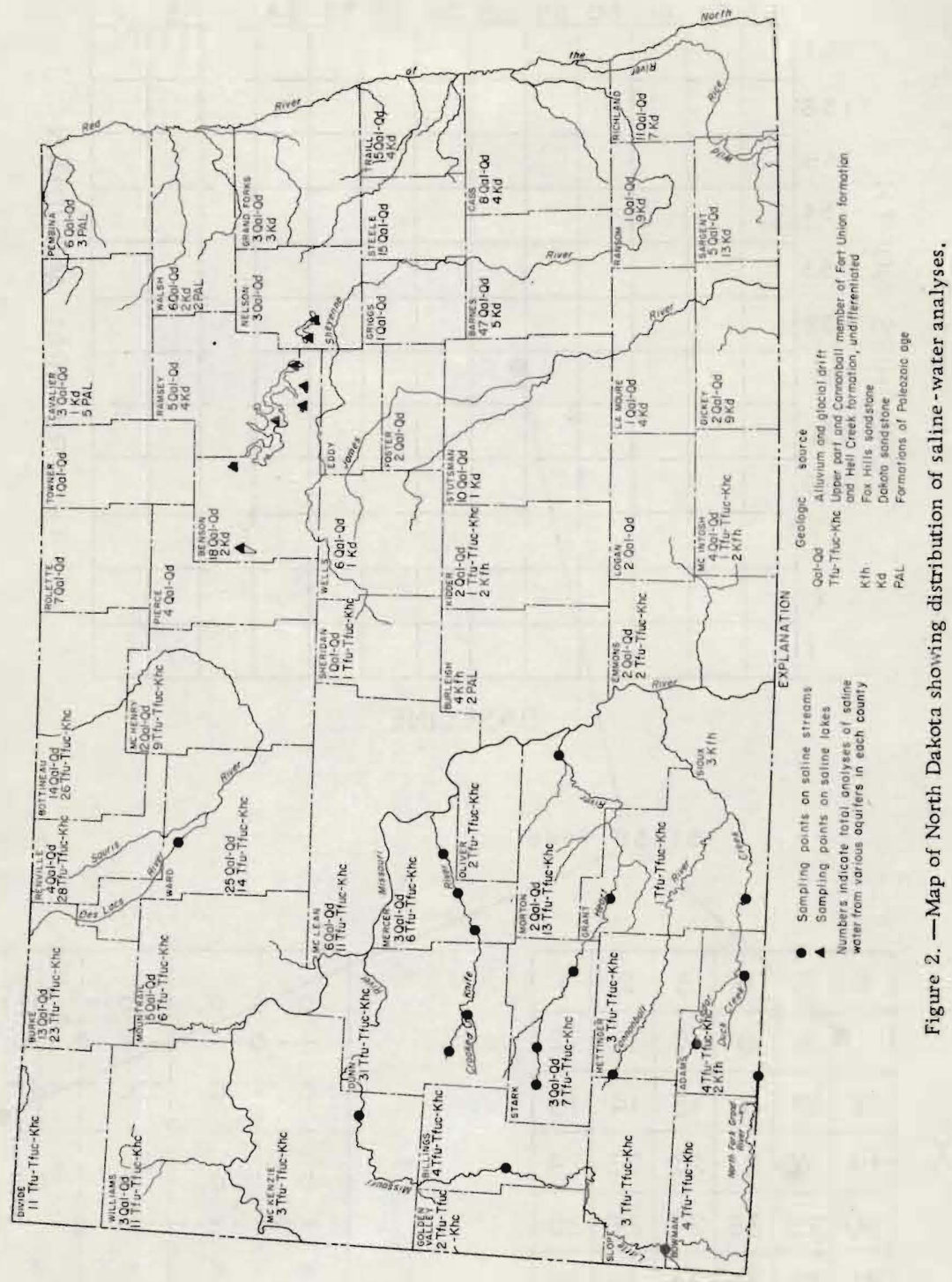


water or saline lakes (connate water), (2) hydrologic interconnection of aquifers containing fresh water with those containing saline water, (3) movement of saline water from a saline-water section of an aquifer to a fresh-water section of the same aquifer as a result of heavy withdrawal of fresh water, (4) solution of mineral constituents in the rocks, (5) seepage of water from saline surface-water bodies into aquifers, and (6) concentration as the result of transpiration by plants and evaporation. Only those processes that apply to North Dakota will be discussed.

In some formations, connate water has become more mineralized through natural processes so that the salinity is greater than that of modern sea water. Such water is found mainly when drilling for oil and gas.

Aquifers containing saline water and those containing fresh water may be connected by natural means, which include depositional contact, juxtaposition by faulting, or fracturing by folding or faulting, so that leakage develops. Water may move between aquifers through confining beds, which are never completely impermeable although they have a permeability lower than that of the aquifers.

Aquifers may be connected also by several artificial means. Wells that have been drilled through both saline-water and freshwater aquifers and that have faulty construction, such as uncemented or corroded and leaky casings or no casings at all, may allow saline water to enter a fresh-water aquifer. A well may tap both a fresh-water aquifer containing water under a relatively high head and a saline-water aquifer containing water under a lower head; pumping such a well may lower the fresh-water head sufficiently to allow water from the saline-water aquifer to enter the fresh-water aquifer. Deep exploration holes for dams and building foundations or other test holes may be drilled through a confining bed and cause leakage from an artesian aquifer containing saline water into an aquifer containing less mineralized water.

Ground water may become saline by solution of mineral constituents in the rocks. Solution takes place as the water percolates downwardfrom the surface and also in the zone of saturation. The total content of dissolved solids in the water is dependent on many factors, such as the solubility of the rocks, the length of time the water is in contact with the rocks, the temperature of the water, and the distance the water travels in the aquifer. If mineralized water comes in contact with ion-exchange minerals in the aquifer, the chemical character of the water may be changed; for example, calcium and magnesium ions, when adsorbed on zeolites, may be replaced by sodium ions. 


\section{SURFACE WATER}

Surface water may become saline by effluent seepage of mineralized ground water, solution of mineral matter by surface runoff, concentration by transpiration and evaporation, and disposal of mineralized wastes into streams.

The salinity of streams and lakes may be due to any combination of the above factors. In western North Dakota those streams that are saline only at base flow probably owe their salinity primarily to seepage of mineralized ground water. In central and eastern North Dakota, uncapped flowing wells may introduce saline water into streams and lakes.

\section{GENERAL GEOLOGY}

North Dakota includes sections of three physiographic regions of the Interior Plains of the United States. (See pl. 1.) These are the Red River Valley, the Drift Prairie, and the Missouri Plateau (Simpson, 1929, p. 4).

The Red River Valley in the eastern part of the State is underlain by sand, silt, and clay that were deposited in glacial Lake Agassiz. These lacustrine deposits rest upon older glacial drift and are bounded by beaches that mark the successive levels of the lake. The drift of Wisconsin age or older rests unconformably on rocks of Paleozoic and, in places, Mesozoic age, which have a gentle dip to the west into the Williston Basin. The rocks of Paleozoic age in turn rest unconformably on the Precambrian complex of igneous and metamorphic rocks. In Grand Forks County the Precambrian rocks are 500 to 900 feet below the land surface.

The Drift Prairie west of the Red River Valleycontains surficial deposits of glacial drift that are continuations of the drift underlying the Lake Agassiz basin. The western boundary of the Drift Prairie is a line east of and approximately parallel to the Missouri River. (See pl. 1.) The Drift Prairie is a region of rolling uplands interspersed with areas of ground moraine and glacial outwash. The most prominent physiographic feature is the Turtle Mountains. Several glacial-lake basins occupy depressions in the drift, notably the basins of glacial Lake Souris in the north-central section of the State and glacial Lake Dakota in the James River valley near the southern boundary of the State. 
Sedimentary rocks of Cretaceous age directly underlie the glacial drift in most parts of the Drift Prairie. The Pierre shale, about 900 feet thick, is the most widely distributed subdrift formation of Cretaceous age in this region. Below the rocks of the Cretaceous system lie older rocks of Mesozoic, Paleozoic, and Precambrian age. (See fig. 3.)

The Missouri Plateau is south and west of the Drift Prairie. A thin veneer of glacial drift mantles the bedrock at the north edge of the Missouri Plateau and ends at the Altamont moraine, which marks the maximum extent of Wisconsin glaciation in North Dakota.

South and west of the Altamont moraine, sedimentary rocks of Late Cretaceous and Tertiary age form the surficial deposits, except in a few places where there is a thin cover of glacial drift older than Wisconsin. The topography of the Missouri Plateau is more rugged than that of the Drift Prairie; large and small buttes form the drainage divides, and in the western counties badlands have formed in the Fort Union formation.

Older rocks of Mesozoic and Paleozoic age overlie Precambrian rocks in the Williston Basin. The center of the basin is in McKenzie County and the sedimentary rocks, which dip into the basin, have only slight structural irregularities (Kunkel, 1954).

\section{AQUIFERS YIELDING SALINE WATER}

Many of the formations shown in figure 3 are capable of yielding only very small supplies of water. The Pierre shale, for example, yields only meager amounts of saline water to many wells in the eastern part of the State. In this report only those aquifers capable of yielding more than $50 \mathrm{gpm}$ to individual wells will be described. (See table 1.) Yields of 50 to $150 \mathrm{gpm}$ are considered small; 150 to $350 \mathrm{gpm}$, moderate; and more than 350 gpm, large.

The chemical characteristics of saline water from various formations and from the glacial drift and alluvium are shown on figures 4 and 5 by means of patterns similar to those proposed by Stiff (1951). The patterns are scaled in equivalents per million of the ions and are useful in comparing different types of water. Figures 4 and 5 show that each major aquifer may yield several types of saline water. 


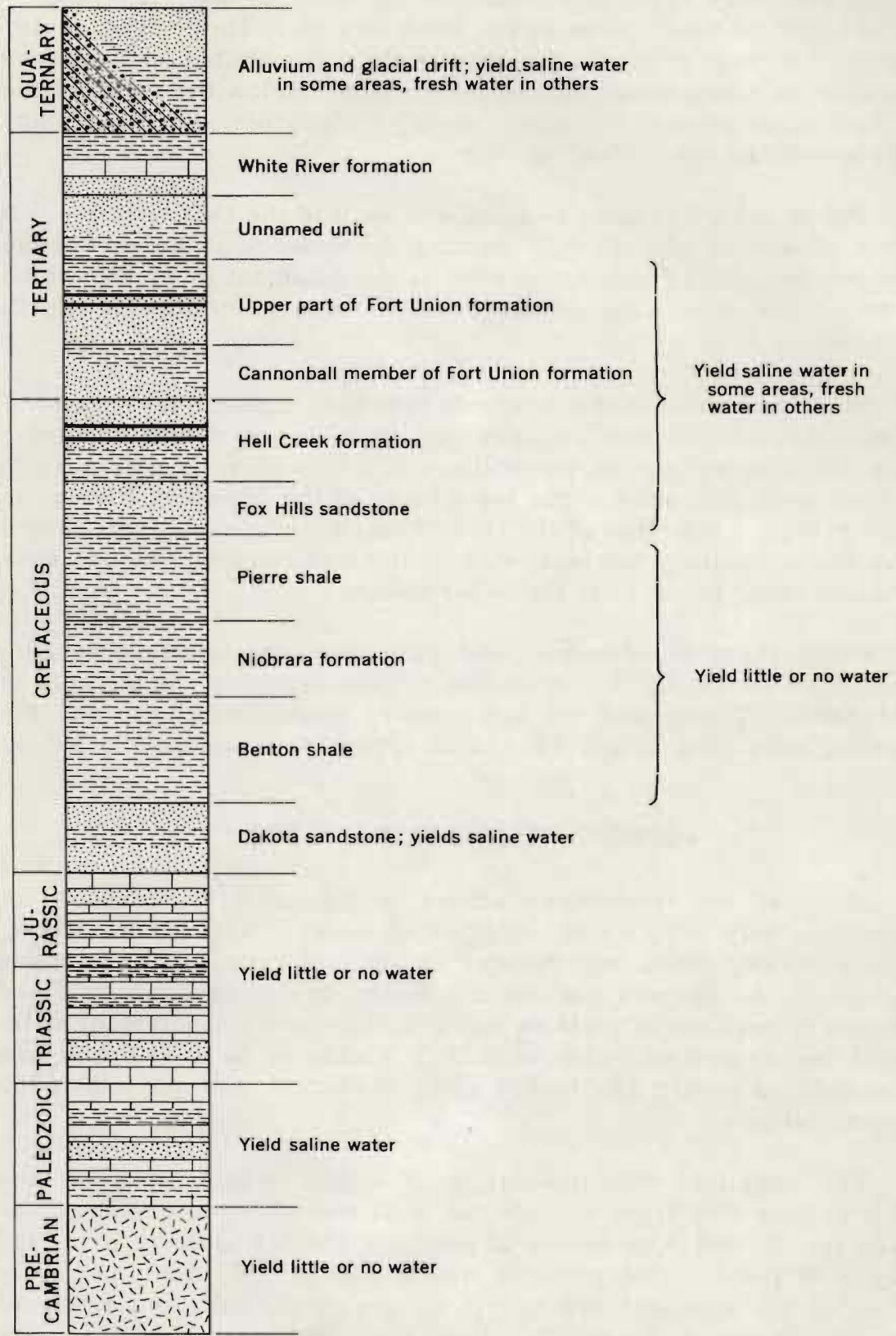

Figure 3. -Generalized stratigraphic section of North Dakota showing saline-water aquifers. Not drawn to scale. 
Table 1.-Description of aquifers of which some parts are capable of yielding 50 gpm or more of saline water to individual wells

\begin{tabular}{|c|c|c|c|c|}
\hline Era & System & Formation & Character of rocks & Saline water supply \\
\hline \multirow{3}{*}{ Cenozoic } & Quaternary & $\begin{array}{l}\text { Alluvium and glacial } \\
\text { drift }\end{array}$ & $\begin{array}{l}\text { Recent: Alluvium; gravel, } \\
\text { sand, silt, and clay. } \\
\text { Pleistocene: Till, glacial } \\
\text { outwash, and glacio- } \\
\text { lacustrine deposits. }\end{array}$ & $\begin{array}{l}\text { Extremely variable } \\
\text { in quantity and } \\
\text { quality. }\end{array}$ \\
\hline & \multirow[b]{2}{*}{ Tertiary } & $\begin{array}{l}\text { Upper part of Fort } \\
\text { Union formation }\end{array}$ & $\begin{array}{l}\text { Sandstone, shale, and } \\
\text { lignite. }\end{array}$ & \multirow{3}{*}{$\begin{array}{l}\text { Small to moderate } \\
\text { supplies in some } \\
\text { areas. }\end{array}$} \\
\hline & & $\begin{array}{l}\text { Cannonball member } \\
\text { of Fort Union } \\
\text { formation }\end{array}$ & $\begin{array}{l}\text { Marine sandstone and } \\
\text { sandy shale. }\end{array}$ & \\
\hline \multirow{3}{*}{ Mesozoic } & \multirow{3}{*}{ Cretaceous } & Hell Creek formation & Sandstone and shale. & \\
\hline & & Fox Hills sandstone & Sandstone. & $\begin{array}{l}\text { Small quantities. } \\
\text { Fresh water in } \\
\text { some areas. }\end{array}$ \\
\hline & & Dakota sandstone & Sandstone and shale. & $\begin{array}{l}\text { Moderate to large } \\
\text { supplies. Artesian } \\
\text { flows in some } \\
\text { areas. }\end{array}$ \\
\hline Paleozoic & & & $\begin{array}{l}\text { Shale, sandstone, lime- } \\
\text { stone, and dolomite. }\end{array}$ & $\begin{array}{l}\text { Small to large sup- } \\
\text { plies in eastern } \\
\text { North Dakota. }\end{array}$ \\
\hline
\end{tabular}

\section{AQUIFERS OF PALEOZOIC AGE}

Formations of Paleozoic age underlie nearly all of North Dakota (see fig. 6) but do not crop out at the surface. The top of the formations lies about 6,000 feet below the land surface in the western part of the State but less than 500 feet below in the eastern part. The formations become thinner and both structurally and topographically higher toward the east: some, and in places perhaps all, are absent in the Red River Valley. Figure 6 indicates by contours on the Dakota sandstone of Cretaceous age the general structure of the rocks of Paleozoic age.

Because the formations are deeply buried, they were penetrated by few wells and were not defined in detail until extensive oil exploration began in the Williston Basin in 1951. Since that time lithologic logs of oil wells and drill-stem tests have provided information about the rock types and about the quality of the water.

Simpson (1929, p. 42) reports saline water from sandstones of Paleozoic age in Pembina and Walsh Counties. Very saline water and brine have been reported from drill-stem tests, mainly in the western part of the State, but data on the quantity of the water are lacking. 


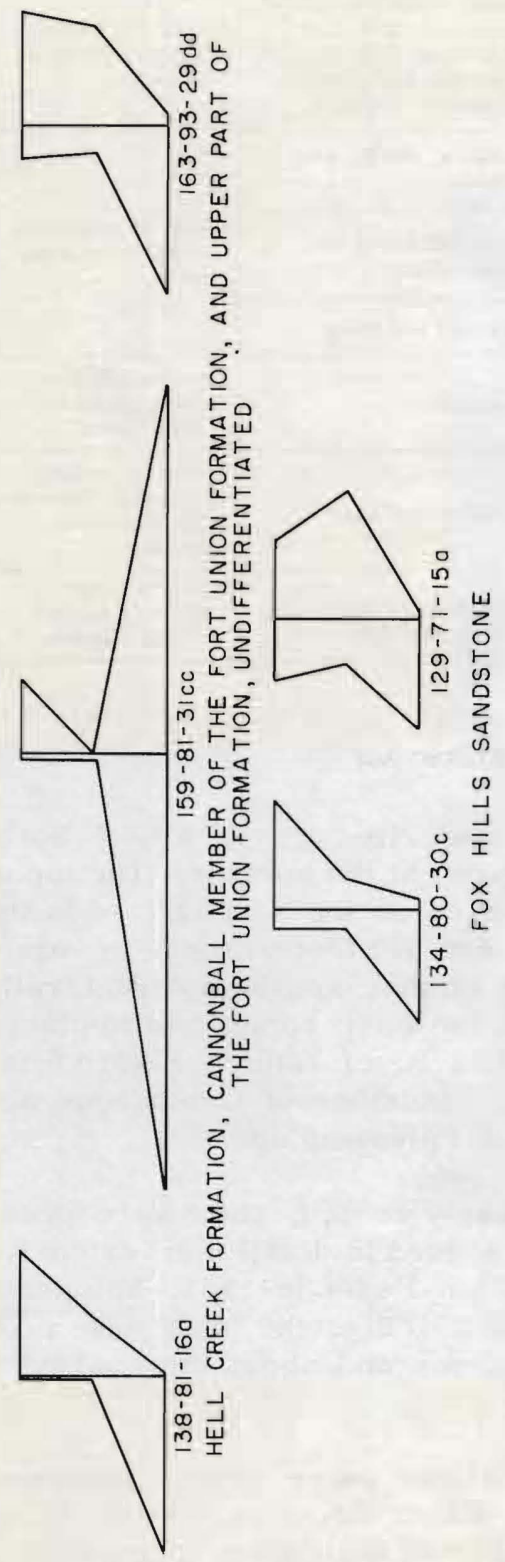

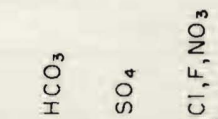

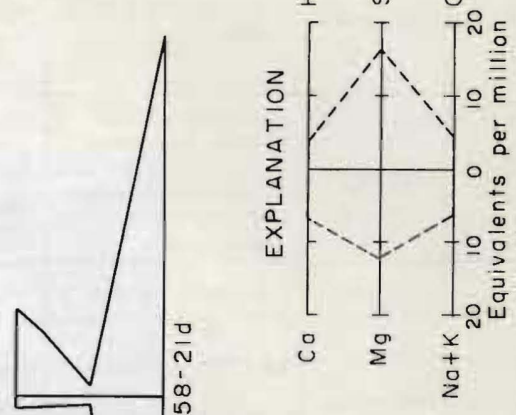

y गु

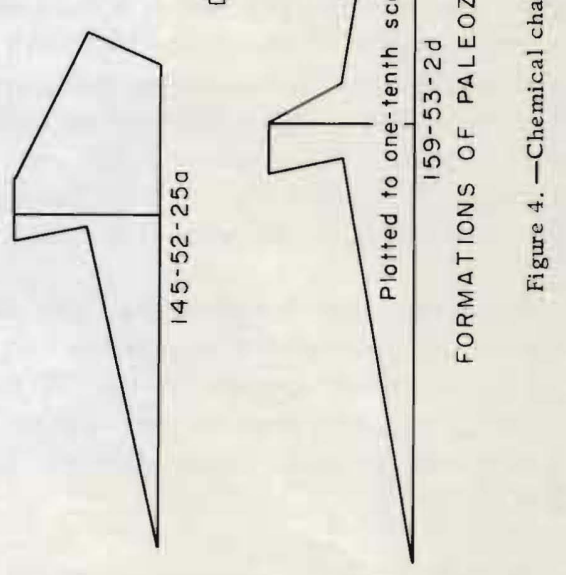



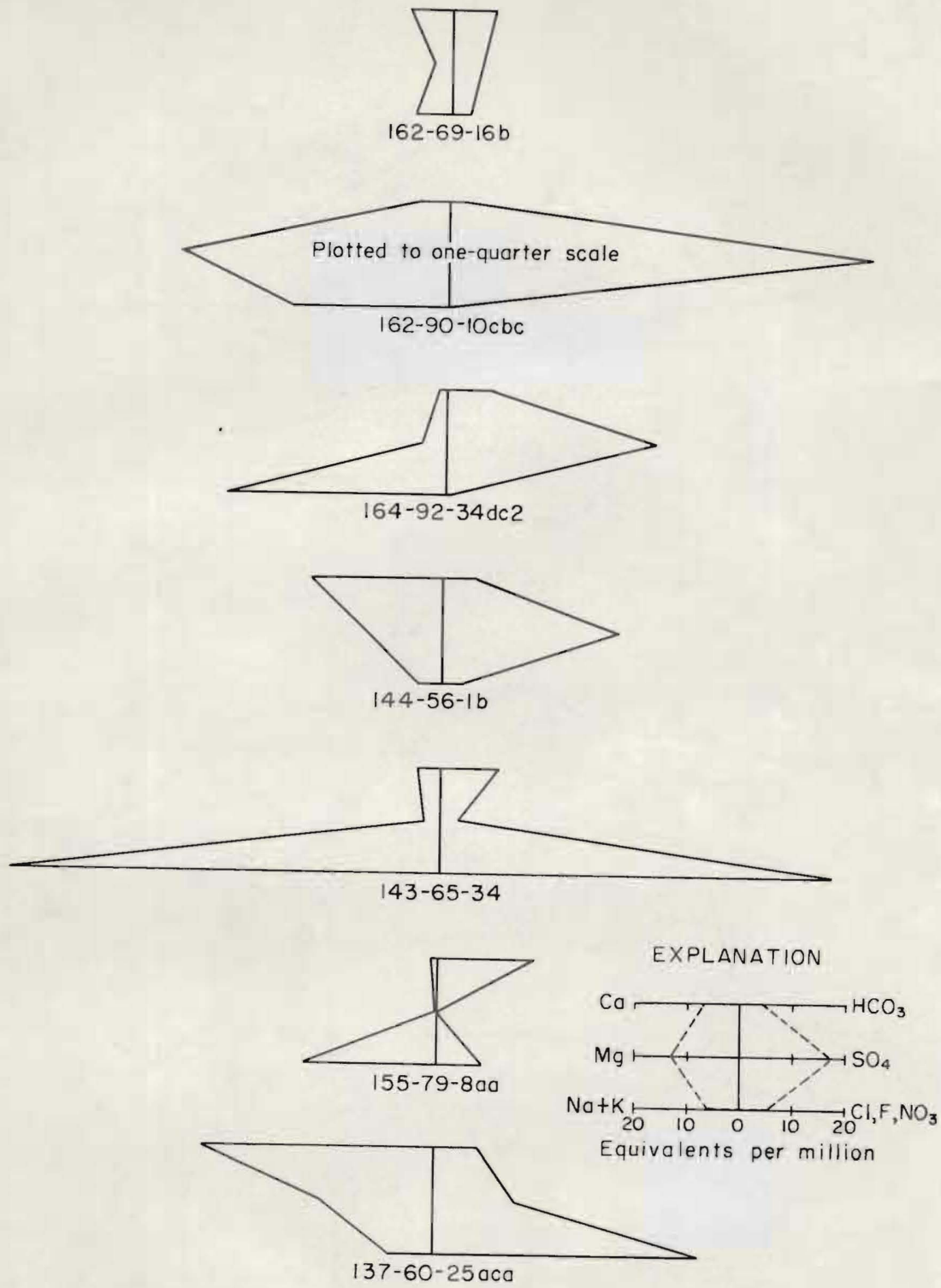

Figure 5. - Chemical characteristics of saline water from glacial drift and alluvium. 


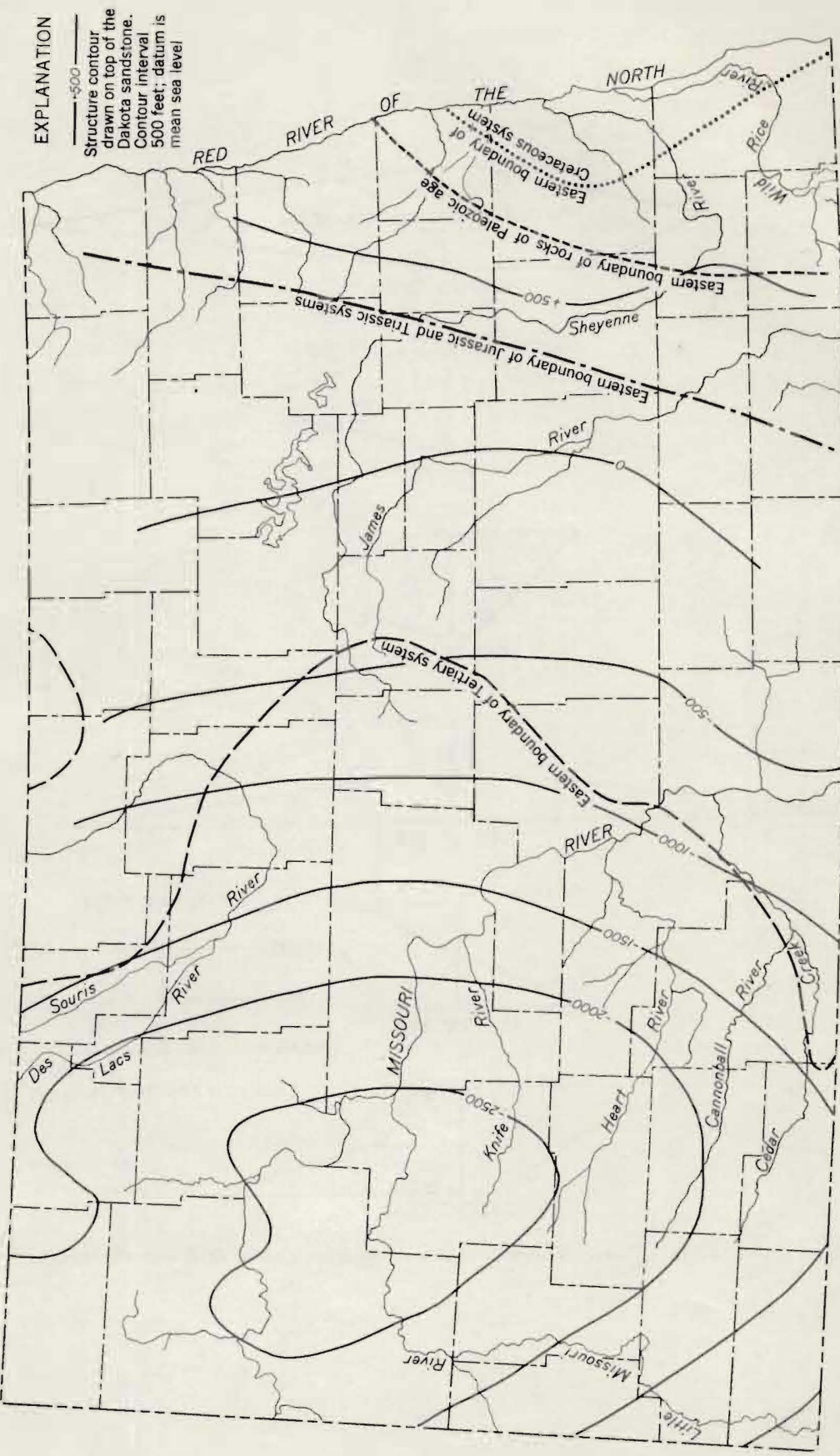


Chemical analyses of saline water from aquifers of Paleozoic age are shown in table 2. Wells 248 to 320 feet deep and 915 feet deep in Walsh County produce moderately saline water, but wells in Pembina, Burleigh, and Cavalier Counties produce brine. Sodium chloride constitutes about 80 percent of the dissolved solids in saline water from these aquifers. The chemical characteristics of water from aquifers of Paleozoic age are compared with those from other sources in figures 4 and 5 . Water from well 159-53-2d was analyzed in 1921 and again in 1954. The change in quality during this 34 -year period was insignificant.

Brine from aquifers of Paleozoic age can be used only for cooling, fire protection, or nonconsumptive uses unless an economical treatment or conversion technique is developed. At Grand Forks, in Grand Forks County, wells drilled to aquifers of Paleozoic age produce up to $400 \mathrm{gpm}$ of very saline water, which is used for air conditioning.

\section{AQUIFERS OF MESOZOIC AND CENOZOIC AGE}

Cretaceous rocks are the most important aquifers of Mesozoic age in North Dakota because rocks of Triassic and Jurassic age are deeply buried and are not known to yield water.

In the major part of the drift-covered area of North Dakota the drift is directly underlain by formations of Cretaceous age. These formations are important aquifers in that part of the drift-covered area where the water from the drift is unsatisfactory in quality or quantity or both.

Saline-water supplies from the Hell Creek formation of Mesozoic age and the Fort Union formation of Cenozoic age are undifferentiated in this report. Although in most of the western part of the State the upper part of the Fort Union formation, which includes the Tongue River and Ludlow members, can be differentiated lithologically from the Cannonball member of the Fort Union formation, the information on available well logs was insufficient to differentiate them for this report.

Alluvium and glacial drift are the most important aquifers of Cenozoic age in the State.

\section{DAKOTA SANDSTONE}

The oldest aquifer of Cretaceous age that yields saline water is the Dakota sandstone, which includes a sandstone unit at the base, 
Table 2.-Chemical analyses of saline ground water

[Source of data: a, U. S. Geological Survey; b, North Dakota Geological Survey

\begin{tabular}{|c|c|c|c|c|c|c|c|c|c|c|}
\hline Location & County & $\begin{array}{l}\text { Depth } \\
\text { of } \\
\text { well } \\
\text { (feet) }\end{array}$ & $\begin{array}{l}\text { Date } \\
\text { of } \\
\text { col- } \\
\text { lection }\end{array}$ & $\begin{array}{l}\text { Tem- } \\
\text { pera- } \\
\text { ture } \\
\left.{ }^{\circ} \mathrm{F}\right)\end{array}$ & $\begin{array}{l}\text { Silica } \\
\left(\mathrm{SiO}_{2}\right)\end{array}$ & $\begin{array}{c}\text { Total } \\
\text { iron } \\
(\mathrm{Fe})\end{array}$ & $\begin{array}{l}\text { Cal- } \\
\text { cium } \\
\text { (Ca) }\end{array}$ & $\begin{array}{c}\text { Mag- } \\
\text { nesium } \\
(\mathrm{Mg})\end{array}$ & $\begin{array}{l}\text { Sodium } \\
\text { (Na) }\end{array}$ & $\begin{array}{l}\text { Potas - } \\
\text { sium } \\
\text { (K) }\end{array}$ \\
\hline
\end{tabular}

Formations of

\begin{tabular}{|c|c|c|c|c|c|c|c|c|c|c|}
\hline & & & 1952 & & & & & & & \\
\hline $140-80-18 \mathrm{ccc}^{1}$ & Burleigh. & 8,115 & Nov, 10.. & .......... & & ........ & 1,558 & 175 & 8,406 & ......... \\
\hline $140-80-18 \operatorname{ccc}^{2}$ & ...do....... & 8,115 & $\begin{array}{c}\text {...do....... } \\
\underline{1921}\end{array}$ & .......... & .......... & $\ldots \ldots$ & 2,830 & 487 & 52,012 & (........... \\
\hline $157-53 \ldots \ldots \ldots$ & Walsh.... & 915 & Apr. 25. & 44 & 24 & $\ldots \ldots$. & $\begin{array}{r}44 \\
1385\end{array}$ & $\begin{array}{r}22 \\
556\end{array}$ & \multirow{2}{*}{\multicolumn{2}{|c|}{$13,450,327$}} \\
\hline $159-53-2 d \ldots$. & Pembina & 450 & $\begin{array}{c}\text {...do....... } \\
1954 \\
\end{array}$ & …........ & 9.4 & 6.6 & & 556 & & \\
\hline $159-53-2 d . . .$. & ...do...... & 450 & Oct. 26.. & 42 & 7.4 & 17 & 1,320 & 535 & 13,600 & 268 \\
\hline $162-53-35 d . .$. & ...do...... & 1,560 & Apr. 23. & 47 & 10 & 10 & 1,285 & 517 & 8,970 & 177 \\
\hline
\end{tabular}

Formations of

\begin{tabular}{|r|r|r|r|r|r|r|r|r|r|}
\hline $157-53^{3} \ldots . .$. & Walsh.... & $248-320$ & May $\frac{1951}{23} . \ldots . . .$. & 11 & 0.78 & 49 & 28 & 1,760 & 18 \\
\hline
\end{tabular}

\begin{tabular}{|c|c|c|c|c|c|c|c|c|c|c|}
\hline \multicolumn{11}{|c|}{ Formations of } \\
\hline $162-64-26 b c^{4}$ & & & $\frac{1951}{\text { Sept. } 20}$ & & & & 38 & & & \\
\hline $162-64-26 b c^{5}$ & ...do...... & $\begin{array}{l}3,380 \\
3,380\end{array}$ & sept. 20. & …........ & (…....... & …....... & $\begin{array}{l}30 \\
15\end{array}$ & & & (…….... \\
\hline $162-64-26 b c^{6}$ & ...do...... & 3,380 & ...do....... & ......... & .......... & $\ldots \ldots$ & 1,825 & 1,125 & ..... & .......... \\
\hline $162-64-26 b c^{7}$ & ...do...... & 3,380 & ...do....... & ......... & .......... & ......... & 3,333 & 2,013 & & \\
\hline $162-64$ & ...do...... & 3,380 & ...do....... & .......... & & & 2,821 & 1,756 & & \\
\hline
\end{tabular}

1 From top of drill stem test.

2 From middle and bottom of drill stem test.

3 Composite sample of water from several wells.

4 Top fluid, drill stem test 1.

a middle unit of shale, and a sandstone unit at the top. The Dakota sandstone as used in this report may include the Fuson shale and Lakota sandstone or their equivalents. Thus, the Dakota is used in a broad sense as Simpson used it in 1929.

The lower sandstone unit is ferruginous; in some places, however, it is white anc micaceous. It yields saline water under artesian head and is sometimes referred to as the "second artesian flow" by well drillers. The shale unit acts as the lower confining bed of the upper unit of sandstone. The upper sandstone unit is similar in lithology to the lower sandstone unit and yields the "first artesian flow." The total thickness of the Dakota sandstone ranges from 150 to 450 feet. 
from formations of Paleozoic age, North Dakota

(Circ. 33 and 57). Analytical results in parts per million except as indicated]

\begin{tabular}{|c|c|c|c|c|c|c|c|c|c|c|c|c|}
\hline \multirow{2}{*}{$\begin{array}{c}\mathrm{Bi}- \\
\text { car- } \\
\text { bon- } \\
\text { ate } \\
\left(\mathrm{HCO}_{3}\right)\end{array}$} & \multirow{2}{*}{$\begin{array}{l}\text { Car- } \\
\text { bon- } \\
\text { ate } \\
\left(\mathrm{CO}_{3}\right)\end{array}$} & \multirow[b]{2}{*}{$\begin{array}{l}\text { Sulfate } \\
\left(\mathrm{SO}_{4}\right)\end{array}$} & \multirow[b]{2}{*}{$\begin{array}{c}\text { Chloride } \\
\text { (Cl) }\end{array}$} & \multirow[b]{2}{*}{$\begin{array}{l}\text { Fluo- } \\
\text { ride } \\
\text { (F) }\end{array}$} & \multirow{2}{*}{$\begin{array}{l} \\
\mathrm{Ni}- \\
\text { trate } \\
\left(\mathrm{NO}_{3}\right)\end{array}$} & \multirow{2}{*}{$\begin{array}{c}\text { Dis- } \\
\text { solved } \\
\text { solids } \\
\text { (residue } \\
\text { on } \\
\text { evapo- } \\
\text { ration } \\
\text { at } \\
180^{\circ} \mathrm{C} \text { ) }\end{array}$} & \multicolumn{2}{|c|}{$\begin{array}{c}\text { Hardness } \\
\text { as } \\
\mathrm{CaCO}_{3}\end{array}$} & \multirow{2}{*}{$\begin{array}{c}\text { Per- } \\
\text { cent } \\
\text { sodi } \\
\text { um }\end{array}$} & \multirow{2}{*}{$\begin{array}{c}\text { Specific } \\
\text { con- } \\
\text { duct- } \\
\text { ance } \\
\text { (micro- } \\
\text { mhos } \\
\text { at } \\
\left.25^{\circ} \mathrm{C}\right)\end{array}$} & \multirow{2}{*}{$\mathrm{pH}$} & \multirow{2}{*}{$\begin{array}{c}\text { Source } \\
\text { of } \\
\text { data }\end{array}$} \\
\hline & & & & & & & $\begin{array}{c}\text { Cal- } \\
\text { cium, } \\
\text { mag- } \\
\text { nesium }\end{array}$ & $\begin{array}{c}\text { Non- } \\
\text { car- } \\
\text { bonate }\end{array}$ & & & & \\
\hline
\end{tabular}

Ordovician age

\begin{tabular}{|c|c|c|c|c|c|c|c|c|c|c|c|c|}
\hline 1,295 & ......... & 2,872 & 12,800 & ....... & ......... & .............. & 4,610 & 3,550 & 80 & ........... & 7.9 & b \\
\hline 120 & ........ & 2,773 & 84,500 & ....... & ......... & .............. & 9,060 & 8,960 & 93 & ............ & 7.15 & b \\
\hline 881 & 0 & 605 & 1,720 & ........ & 9.0 & 4,560 & 200 & & 95 & & & a \\
\hline 223 & 0 & 2,557 & 23,370 & ........ & ....... & 42,345 & 5,740 & 5,560 & 83 & & ..... & a \\
\hline 201 & 0 & 2,470 & 23,300 & 1.6 & ........ & 44,300 & 5,490 & 5,330 & 84 & 57,400 & 7.0 & a \\
\hline 174 & 0 & 3,714 & 15,050 & & 4.0 & 30,290 & 5,330 & 5,190 & 78 & & & a \\
\hline
\end{tabular}

Silurian age(?)

\begin{tabular}{|c|c|c|c|c|c|c|c|c|c|c|c|c|}
\hline 867 & 0 & 618 & 1,940 & 2.8 & 18 & 4,880 & 238 & 0 & 94 & 7,980 & 7.5 & a \\
\hline
\end{tabular}

Devonian age

\begin{tabular}{|c|c|c|c|c|c|c|c|c|c|c|c|c|}
\hline 156 & 52 & 969 & 473 & & & 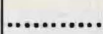 & & & & & & b \\
\hline 164 & 65 & 996 & 489 & & ....... & ............... & ............... & ............. & ....... & .............. & .... & b \\
\hline 116 & ........ & 2,044 & 19,311 & $\ldots$ & ......... & ................ & 9,180 & 9,090 & & ............. & ..... & b \\
\hline 918 & ........ & 1,127 & 40,551 & ........ & $\mid . . . . .$. & ............... & 16,600 & 15,800 & ........ & ................ & |..... & b \\
\hline 75 & ....... & 2,396 & 34,748 & ....... & ......... & ................ & 14,300 & 14,200 & & .............. & $\cdots \cdot$ & b \\
\hline
\end{tabular}

5 Bottom fluid, drill stem test 1.

6 Top fluid, drill stem test 2 .

7 Middle fluid, drill stem test 2.

8 Bottom fluid, drill stem test 2.

The Dakota sandstone supplies flowing wells in a large area in southeastern and central North Dakota (Simpson, 1929, pl. 1). The area of flowing wells has been considerably reduced in size, and the artesian pressure of the water has been reduced, as the result of the discharge of many flowing wells. Many wells that at one time flowed at high pressure now flow under reduced pressure or do not flow at all and have to be pumped.

The quality of saline water from the Dakota sandstone is represented by 69 analyses in table 3. Generally, in the southeastern part of the State, the water is slightly saline; however, north of about T. $144 \mathrm{~N}$., the water is moderately saline. A well (151-5015b) near Grand Forks produces very saline water, as does a well (161-60-23) at Langdon. 


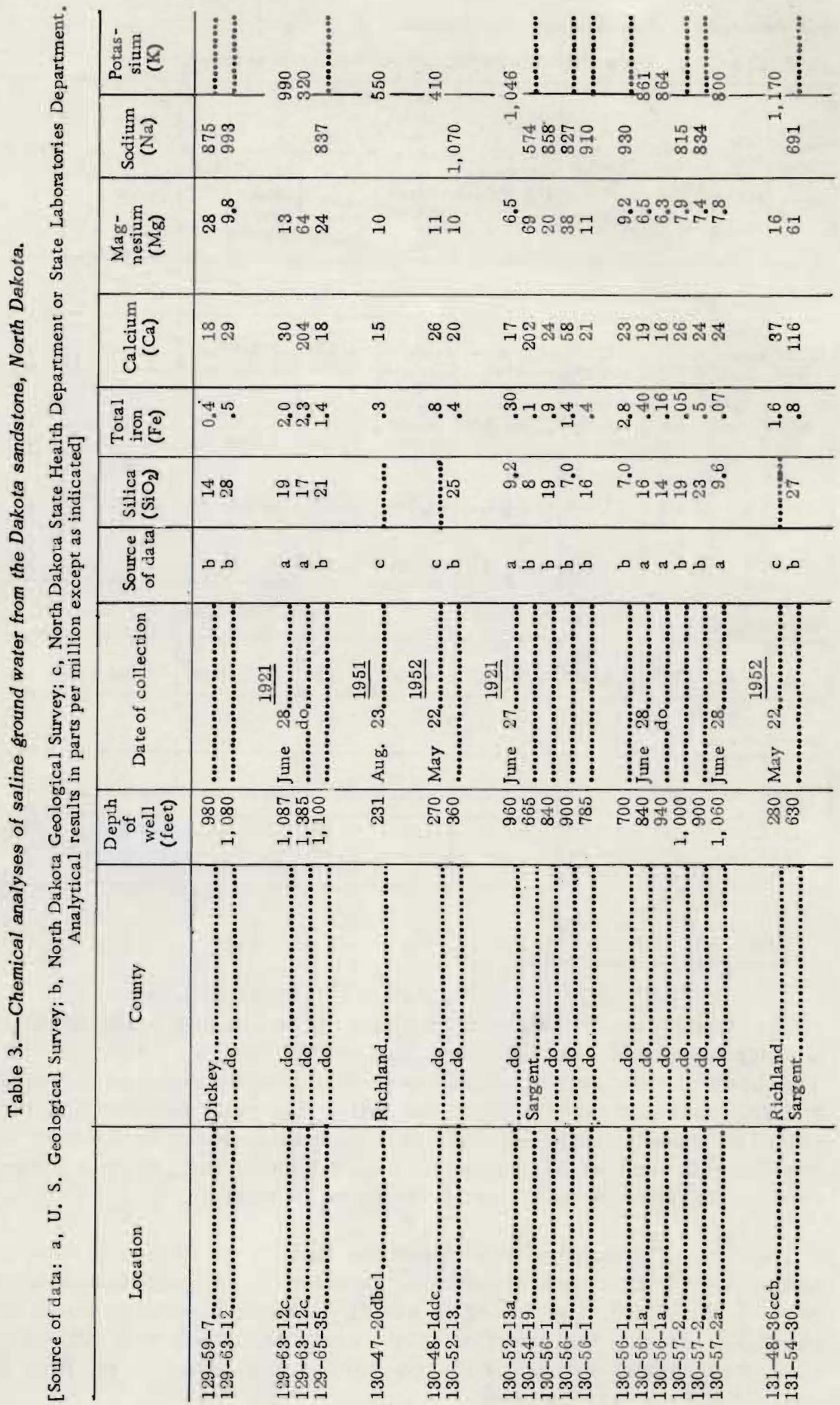




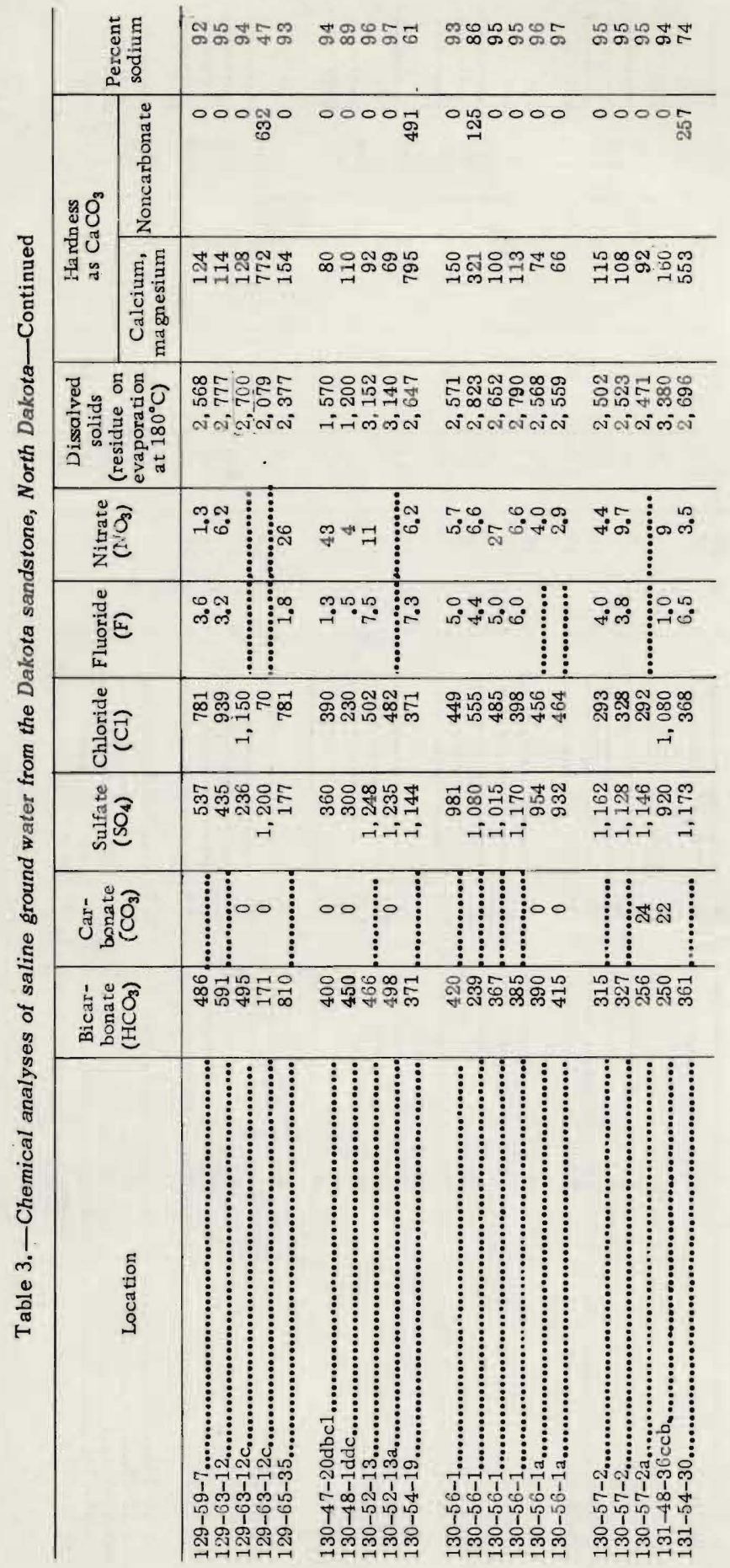




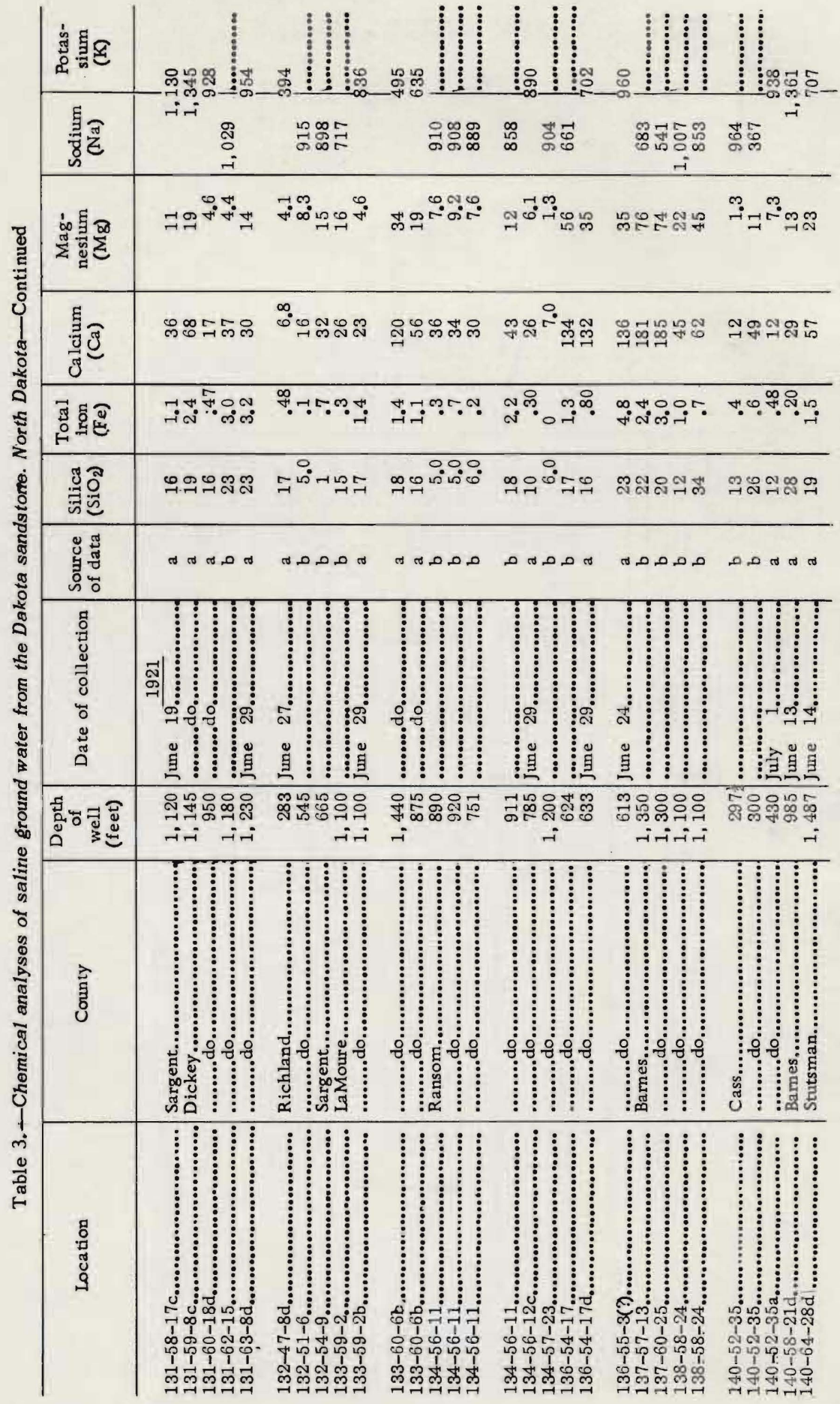




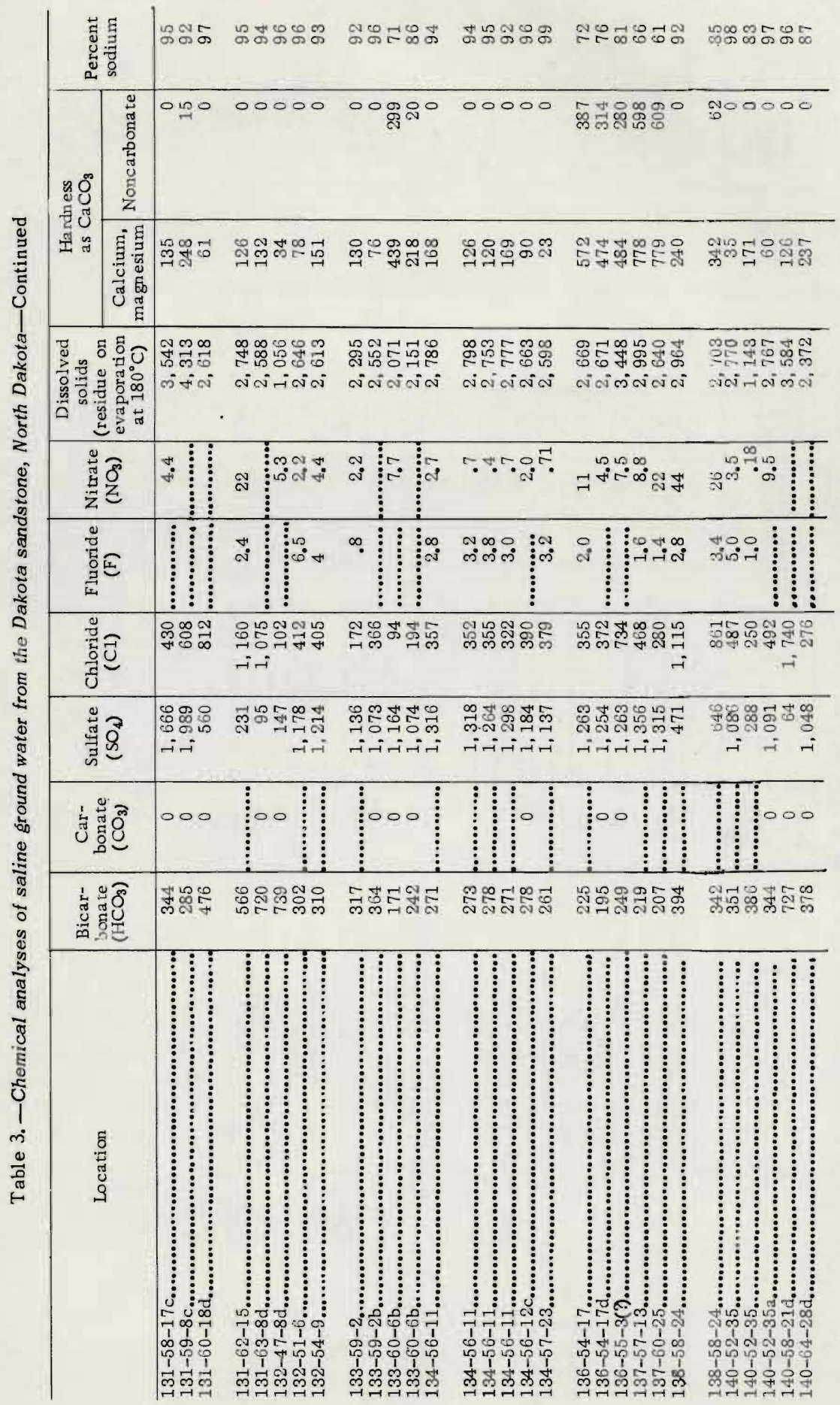




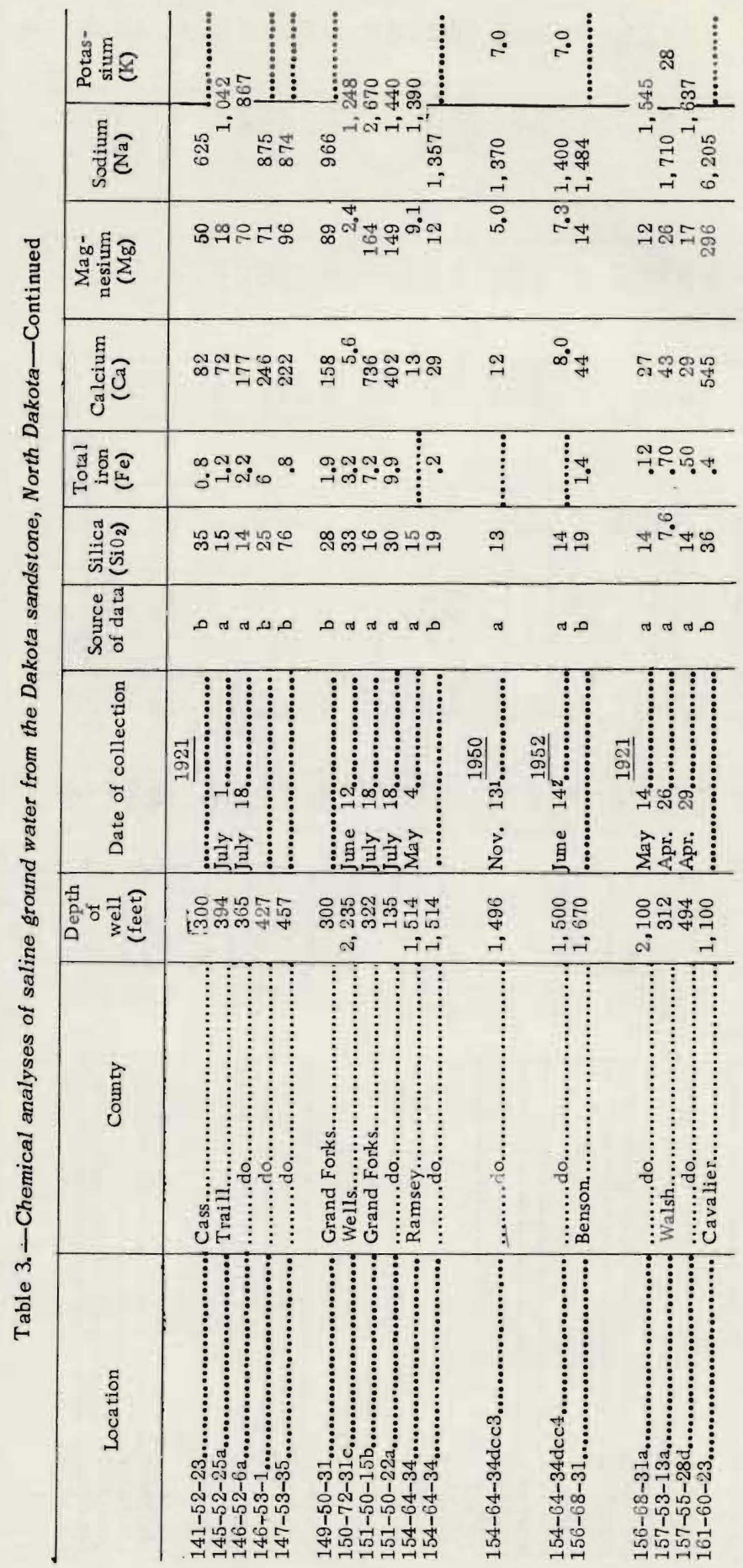




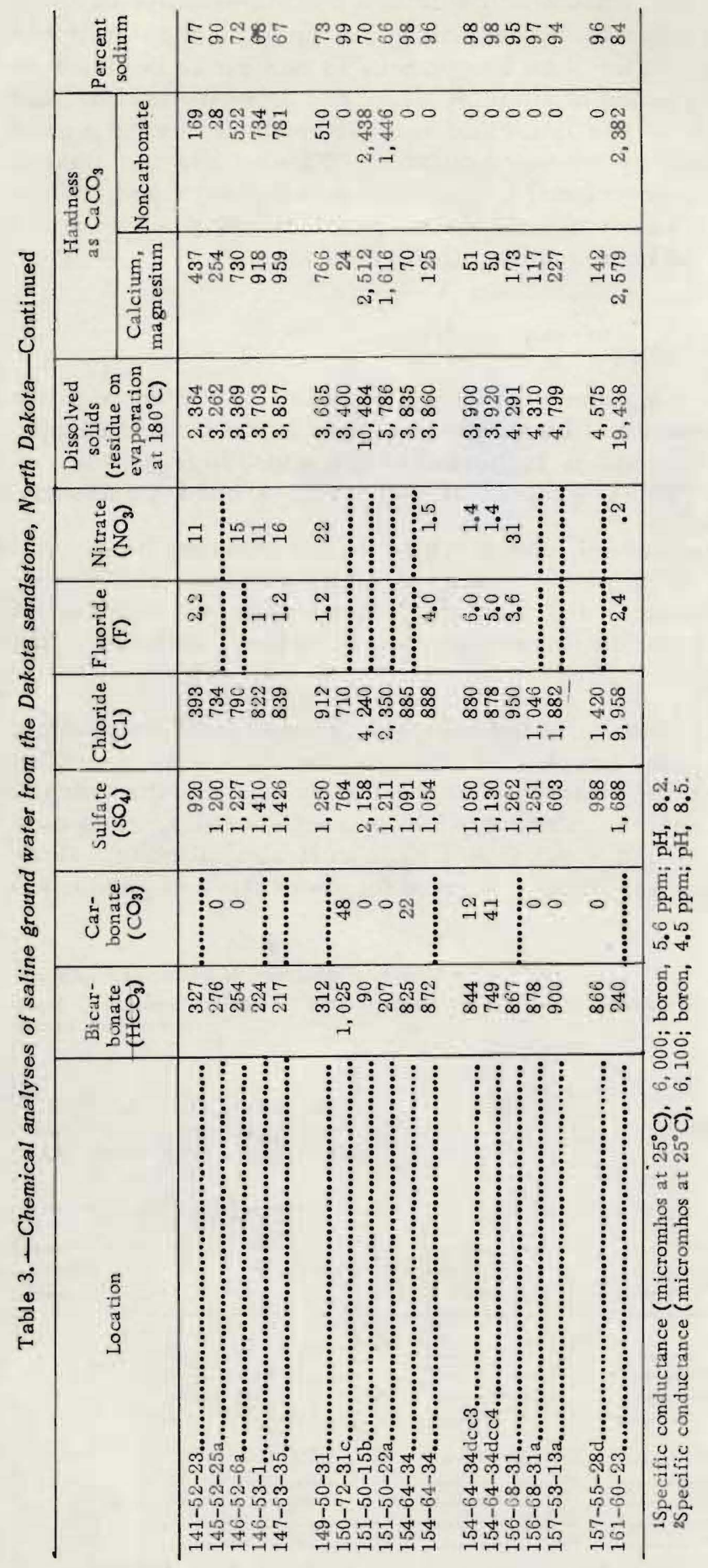


Sodium sulfate and sodium chloride are the major salts in solution. (See fig. 4.) Hardness exceeded $200 \mathrm{ppm}$ in about half the samples and was greater than $500 \mathrm{ppm}$ in 13 samples. Because of excessive concentrations of fluoride, iron, and dissolved solids, the water is undesirable for domestic use, although it is used where water of better quality is not available. Water from the Dakota sandstone is unsuitable for irrigation because of high sodium and dissolved-solids concentrations; furthermore, in few places would the supply be adequate in quantity for irrigation.

\section{FOX HILLS SANDSTONE}

The Fox Hills sandstone directly overlies the Pierre shale throughout the Missouri Plateau and in places in the Drift Prairie. In the Missouri Plateau it is between 180 and 320 feet in thickness, but in the Drift Prairie it is partly eroded or absent.

Fresh water is obtained from the Fox Hills sandstone at several places, including Hettinger in Adams County, Bowman and Scranton in Bowman County, and Lemmon, S. Dak. Small supplies of saline water are found in Adams, Burleigh, Kidder, McIntosh, and Sioux Counties.

Water from the Fox Hills sandstone is primarily of the sodium bicarbonate type. (See fig. 4.) Twelve analyses of saline water in table 4 show that the water is only slightly saline; the average dissolved-solids content is about $1,500 \mathrm{ppm}$. Generally, the water in Adams, Burleigh, and Sioux Counties is soft; and, although fluoride concentrations are high, it is used for domestic and municipal

Table 4. - Chemical analyses of saline ground water [Source of data: a, U. S. Geological Survey; b, North Dakota Geological Survey; c, North

\begin{tabular}{|c|c|c|c|c|c|c|c|c|c|}
\hline Location & County & $\begin{array}{l}\text { Depth } \\
\text { of } \\
\text { well } \\
\text { (feet) }\end{array}$ & $\begin{array}{l}\text { Date } \\
\text { of } \\
\text { collection }\end{array}$ & $\begin{array}{l}\text { Silica } \\
\left(\mathrm{SiO}_{2}\right)\end{array}$ & $\begin{array}{l}\text { Total } \\
\text { iron } \\
\text { (Fe) }\end{array}$ & $\begin{array}{l}\text { Cal- } \\
\text { cium } \\
\text { (Ca) }\end{array}$ & $\begin{array}{l}\text { Mag- } \\
\text { nesium } \\
(\mathrm{Mg})\end{array}$ & $\begin{array}{l}\text { Sodi- } \\
\text { um } \\
(\mathrm{Na})\end{array}$ & $\begin{array}{l}\text { Potas- } \\
\text { sium } \\
(\mathrm{K})\end{array}$ \\
\hline $\begin{array}{l}129-71-10 . . . . . . . \\
129-71-15 \text {. ....... } \\
129-96-13 . . . . . . . \\
129-96-13 \text { bьь.... }\end{array}$ & $\begin{array}{l}\text { McIntosh....... } \\
\ldots . . . \text { do............ } \\
\text { Adams .......... } \\
\ldots . . . \text { do.......... }\end{array}$ & $\begin{array}{r}130 \\
146 \\
1,152 \\
1,150\end{array}$ & 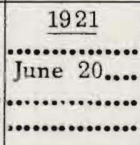 & $\begin{array}{c}44 \\
37 \\
15 \\
\ldots \ldots \ldots\end{array}$ & $\begin{array}{c}1.1 \\
0.80 \\
.7\end{array}$ & $\begin{array}{r}108 \\
168 \\
2.4 \\
\ldots \ldots \ldots\end{array}$ & $\begin{array}{c}48 \\
76 \\
4.5 \\
\ldots \ldots . .\end{array}$ & $\mid \begin{array}{l}409 \\
423 \\
418\end{array}$ & $\begin{array}{l}\mid \ldots \ldots \ldots \\
346 \\
1.8 \ldots\end{array}$ \\
\hline $\begin{array}{l}134-80-30 \text { c........ } \\
134-80-30 \text { c....... } \\
134-80-30 \text { c........ } \\
138-80-4 \text { b........ }\end{array}$ & $\begin{array}{l}\text { Sioux............ } \\
\text {....do............ } \\
\text { B...do........... } \\
\text { Burleigh....... }\end{array}$ & $\begin{array}{l}67 \\
65 \\
65 \\
75\end{array}$ & $\begin{array}{c}\text { June } 27 . . . \\
\ldots . . . \text { do.......... } \\
\ldots . . . \text { do........ } \\
\text { June } 16 . . .\end{array}$ & $\begin{array}{l}17 \\
21 \\
16 \\
17\end{array}$ & $\begin{array}{r}.63 \\
.20 \\
.15\end{array}$ & $\begin{array}{l}8.0 \\
12 \\
46 \\
15\end{array}$ & $\begin{array}{c}2.6 \\
4.6 \\
32 \\
2.4\end{array}$ & & $\begin{array}{r}520 \\
580 \\
350 \\
540\end{array}$ \\
\hline 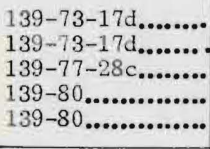 & 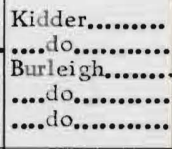 & $\begin{array}{l}120 \\
110 \\
125 \\
200 \\
400\end{array}$ & $\begin{array}{l}\text { June } 15 \ldots . . . \\
\ldots . . . \text { do......... } \\
\text { June } 16 \ldots . . . . \\
\ldots . . . \text { do......... }\end{array}$ & $\begin{array}{l}46 \\
45 \\
19 \\
13 \\
37\end{array}$ & $\begin{array}{r}.72 \\
\ldots \ldots \ldots \\
4.0 \\
. . .0 \\
.27\end{array}$ & $\begin{array}{l}150 \\
193 \\
476 \\
7.4 \\
9.6\end{array}$ & $\begin{array}{r}62 \\
65 \\
814 \\
2.3 \\
2.8\end{array}$ & $\begin{array}{r}1 \\
2,3 \\
6 \\
9 \\
\end{array}$ & $\begin{array}{r}101 \\
55 \\
362 \\
259 \\
51 \\
\end{array}$ \\
\hline
\end{tabular}


supplies. Farther east, in McIntosh and Kidder Counties, saline water from the Fox Hills sandstone is harder and has a lower percent sodium and thus is more suitable for irrigation.

Well 139-77-28c produces a very saline water of the sodium sulfate type. The well is in a poorly drained area, and the high salinity of the water may be a result of concentration by evaporation (Simpson, 1929, p. 96).

\section{HELL CREEK FORMATION AND FORT UNION FORMATION, UNDIFFERENTIATED}

The Hell Creek formation and the Cannonball member and the upper part of the Fort Union formation crop out in a major part of the Missouri Plateau in North Dakota. The Tongue River member, which together with the Ludlow member constitutes the upper part of the Fort Union formation, is widely distributed in the plateau uplands; but the stratigraphically lower Hell Creek formation and Cannonball member of the Fort Union crop out only in the banks of the Missouri River and its tributaries south of Washburn and in the Little Missouri River basin in the southwestern corner of the State.

These aquifers are discussed together because of the difficulty in differentiating between them by means of well logs. Because of lateral discontinuity of the beds and pinchouts of aquifers between confining beds of shale, the ayuifers may be dewatered or water levels lowered by heavy or prolonged pumping.

Chemical analyses of saline water from these aquifers are given in table 5 . Generally, the water is only slightly saline.

from the Fox Hills sandstone, North Dakota

Dakota State Health Department or State Laboratories Department. Analytical results in parts as indicated]

\begin{tabular}{|c|c|c|c|c|c|c|c|c|c|c|c|}
\hline \multirow{2}{*}{$\begin{array}{l}\text { Bicar- } \\
\text { bonate } \\
\left(\mathrm{HCO}_{3}\right)\end{array}$} & \multirow{2}{*}{$\begin{array}{l}\text { Car- } \\
\text { bonate } \\
\left(\mathrm{CO}_{3}\right)\end{array}$} & \multirow[b]{2}{*}{$\begin{array}{l}\text { Sulfate } \\
\left(\mathrm{SO}_{4}\right)\end{array}$} & \multirow{2}{*}{$\begin{array}{l}\text { Chlo- } \\
\text { ride } \\
\text { (Cl) }\end{array}$} & \multirow{2}{*}{$\begin{array}{l}\text { Fluo- } \\
\text { ride } \\
\text { (F) }\end{array}$} & \multirow{2}{*}{$\begin{array}{c}\text { Nitra te } \\
\left(\mathrm{NO}_{3}\right)\end{array}$} & \multirow{2}{*}{$\begin{array}{l}\text { Dissolved } \\
\text { solids } \\
\text { (residue } \\
\text { on evapo- } \\
\text { ration } \\
\text { at } 180^{\circ} \mathrm{C} \text { ) }\end{array}$} & \multicolumn{2}{|c|}{$\begin{array}{l}\text { Hardness } \\
\text { as } \mathrm{CaCO}_{3}\end{array}$} & \multirow{2}{*}{$\begin{array}{l}\text { Per- } \\
\text { cent } \\
\text { so- } \\
\text { dium }\end{array}$} & \multirow[b]{2}{*}{$\mathrm{pH}$} & \multirow{2}{*}{$\begin{array}{c}\text { Sourc } \\
\text { of } \\
\text { data }\end{array}$} \\
\hline & & & & & & & $\begin{array}{l}\text { Cal- } \\
\text { cium, } \\
\text { ma gne } \\
\text { sium }\end{array}$ & $\begin{array}{l}\text { Non- } \\
\text { carbon- } \\
\text { ate }\end{array}$ & & & \\
\hline 597 & & 702 & 94 & 0.8 & 4.9 & 1.700 & 469 & 0 & 66 & & b \\
\hline 671 & 0 & 848 & 16 & $\ldots .$. & 3.0 & 1,905 & 732 & 182 & 51 & $\ldots$ & a \\
\hline 894 & & 24 & 128 & 3.2 & .3 & 1,070 & 28 & 0 & 97 & $\ldots . .$. & b \\
\hline 368 & 57 & 45 & 130 & 3.5 & 6 & 1.096 & 90 & 0 & 91 & 8.7 & c \\
\hline 1,039 & 0 & 227 & 60 & 1....... & 10 & 1,367 & 31 & 0 & 97 & & a \\
\hline 1,078 & 0 & 346 & 47 & & 7.4 & 1,539 & 49 & 0 & 96 & $\ldots$ & a \\
\hline 903 & 0 & 200 & 45 & & 24 & 1,168 & 246 & 0 & 76 & ..... & a \\
\hline 1,015 & 0 & 323 & 64 & & 3.0 & 1,509 & 47 & 0 & 96 & $\ldots$ & a \\
\hline 561 & 0 & 366 & 6.0 & & & 1,038 & 629 & 169 & 26 & & a \\
\hline 554 & 0 & 389 & 4.0 & & .33 & 1,059 & 749 & 295 & 14 & $\ldots .$. & a \\
\hline 1,188 & 0 & 7,838 & 386 & .......... & 7.2 & 13,807 & 4,530 & 3,560 & 53 & ..... & a \\
\hline 1,035 & 0 & 491 & 66 & .......... & 1.7 & 1,776 & 28 & 0 & 98 & $\ldots$ & a \\
\hline 1,174 & 0 & 5.9 & 824 & .......... & ........... & 2,441 & 35 & 0 & 98 & $\ldots$ & a \\
\hline
\end{tabular}


Table 5.-Chemical analyses of saline ground water from the Hell Creek formation, formation, undifferentiated,

[Source of data: a, U. S. Geological Survey: b, North Dakota Geological

\begin{tabular}{|c|c|c|c|c|c|c|c|c|c|c|}
\hline Location & County & $\begin{array}{l}\text { Depth } \\
\text { of } \\
\text { well } \\
\text { (feet) }\end{array}$ & $\begin{array}{l}\text { Date } \\
\text { of } \\
\text { col- } \\
\text { lection }\end{array}$ & $\begin{array}{l}\text { Tem- } \\
\text { pera- } \\
\text { ture } \\
\left({ }^{\circ} \mathrm{F}\right)\end{array}$ & $\begin{array}{l}\text { Silica } \\
\left(\mathrm{SiO}_{2}\right)\end{array}$ & $\begin{array}{r}\text { Total } \\
\text { iron } \\
(\mathrm{Fe})\end{array}$ & $\begin{array}{l}\text { Cal- } \\
\text { cium } \\
(\mathrm{Ca})\end{array}$ & $\begin{array}{c}\text { Mag- } \\
\text { ne- } \\
\text { sium } \\
(\mathrm{Mg})\end{array}$ & $\begin{array}{l}\text { Sodium } \\
\text { (Na) }\end{array}$ & $\begin{array}{l}\text { Potas - } \\
\text { sium } \\
(K)\end{array}$ \\
\hline $129-94-29 \ldots \ldots$ & Adams. & 18 & & & 16 & 0.30 & 188 & 170 & 310 & \\
\hline $129-94-29 \ldots \ldots$ & ....do... & 120 & & & 10 & .80 & 47 & 32 & 913 & \\
\hline $130-74-31 \ldots .$. & Emmons.... & 120 & & & 27 & .8 & 162 & 51 & 297 & .......... \\
\hline $130-97-23 \ldots$. & Adams...... & 53 & ............ & ......... & 18 & .30 & 93 & 145 & 256 & ........... \\
\hline $130-98-4 \ldots \ldots$. & ....da......... & 72 & ......... & & 8.0 & 6.0 & 160 & 103 & 120 & …...... \\
\hline $131-99-33 \ldots .$. & Bowman.... & 75 & .............. & & 9 & 6.0 & 72 & 55 & 232 & \\
\hline $131-100-26 \ldots$ & ....do.......... & 98 & ............. & $\ldots .$. & 6 & 0 & 30 & .65 & 453 & $\cdots$ \\
\hline $131-102-11 \ldots$ & ...do......... & 1,042 & .............. & ........ & 4 & 0 & 3.3 & 4.1 & 359 & .......... \\
\hline $132-69-6 \ldots \ldots$ & McIntosh... & 254 & n............ & ......... & 42 & .7 & 144 & 35 & 204 & .......... \\
\hline $132-104-27 \ldots$. & Bowman.... & 25 & ................ & & 6 & 1.1 & 112 & 85 & 215 & ........... \\
\hline $133-105-31$ & Slope.. & 200 & July 22 s: & & 17 & .69 & 9.6 & 3.3 & 570 & \\
\hline $133-106-3$ & ....do... & 185 & ................ & & 2 & .1 & 10 & 4.8 & 584 & .......... \\
\hline $134-84-4 \ldots \ldots .$. & Morton. & 200 & .............. & & 21 & .5 & 30 & 33 & 420 & .......... \\
\hline $\begin{array}{l}134-90-35 \ldots . . \\
134-93-35 \ldots\end{array}$ & Grant... & $\begin{array}{l}431 \\
378\end{array}$ & ..... & $\cdots$ & 23 & .02 & 7.8 & $\begin{array}{l}8.2 \\
0.8\end{array}$ & 640 & .......... \\
\hline $134-93-35 \ldots .$. & Hetting & 378 & ........... & & 17 & 0 & 5.1 & 2.8 & 502 & |........... \\
\hline $134-93-35 c \ldots$ & ....do.......... & 177 & June $28 .$. & & 14 & .80 & 7.6 & 4.3 & 430 & \\
\hline $135-76-30 \ldots$. & Emmons.... & 185 & & & 25 & .3 & 16 & 12 & 400 & \\
\hline $135-97-4 \ldots \ldots$ & Hettinger... & 78 & ............... & $\ldots \ldots$ & 12 & .5 & 63 & 9.0 & 311 & .......... \\
\hline $135-101-26 \ldots$ & Slope.... & 265 & n........... & & 134 & .8 & 10 & 8.1 & 398 & ......... \\
\hline $137-84-10 c c .$. & Morton. & 47.4 & Sept. 17.. & & & ….... & 140 & 68 & 133 & \\
\hline $138-81-16 a \ldots$ & ....do. & 400 & Sept. 22. & 49 & 14 & .32 & 5.3 & 3.9 & 565 & 2.2 \\
\hline $138-81-16$ & ....do.. & 318 & $\frac{1921}{\text { June } 20}$ & & 14 & .07 & 4.0 & 3.1 & 520 & \\
\hline $139-81-27 \ldots \ldots$ & ....do.......... & $400-500$ & ....do.... & & 28 & .20 & 18 & 7.6 & 795 & \\
\hline $139-81-33 c \ldots$ & ....do......... & 330 & ....do.... & .......... & 15 & .40 & 5.6 & 2.7 & 575 & \\
\hline $139-85-21 \ldots .$. & ....do......... & 365 & ............ & & 23 & 3.0 & 77 & 76 & 225 & - $\cdots \cdots \cdots$ \\
\hline $139-85-21 \ldots .$. & ....do......... & 70 & $\cdots \cdots$ & & 17 & 0 & 250 & 112 & 89 & $\cdots \cdots \cdots$ \\
\hline $139-85-21 \ldots$. & ....do... & 56 & ...... & ..... & 24 & .6 & 13 & 12 & 580 & \\
\hline $139-88-31 \ldots .$. & ....do......... & 78 & ............. & (....... & 26 & .1 & 67 & 41 & 272 & .......... \\
\hline $\begin{array}{l}139-92-5 \ldots \ldots . \\
139-95-3 \ldots \ldots .\end{array}$ & Stark........ & 110 & ............. & (........ & 14 & 2.2 & 64 & 51 & 235 & ........... \\
\hline $139-90-3 \ldots . .$. & ...do........ & 200 & (n....... & & 12 & .8 & 91 & 66 & 287 & ........... \\
\hline $139-96-4 a c 1 .$. & ....do......... & 154.0 & $\frac{1947}{\text { July } 18}$ & & 12 & .10 & 9.0 & .7 .0 & 574 & 6.4 \\
\hline 139-96-10dd.. & ....do......... & 120 & ....do..... & $\ldots \ldots$ & 11 & 8.0 & 107 & 59 & 508 & 12 \\
\hline $139-96-12 \mathrm{bb} .$. & ....do......... & 68 & Oct. 6. & & ....... & .30 & 82 & 49 & 949 & 9 \\
\hline $139-99-5 \ldots \ldots$ & ....do......... & 230 & …............ & ........ & 11 & .2 & 6.4 & 13 & $475 \quad$. & $|\ldots . . . . .|$. \\
\hline $139-104-5 c \ldots$ & $\begin{array}{l}\text { Golden } \\
\text { Valley. }\end{array}$ & Spring & $\frac{1921}{\text { July } 20 \ldots}$ & & 27 & .70 & 44 & 24 & 645 & \\
\hline $140-90-28 \ldots .$. & Morton..... & 434 & & & 15 & .6 & 19 & 13 & 633 & \\
\hline $140-90-28 \ldots \ldots$ & ....do......... & 238 & & & 20 & .5 & 9.7 & 23 & $633 \mid$. & \\
\hline $140-90-33 \mathrm{~b} . .$. & ....do......... & 215 & June 23.. & ........ & 19 & .68 & 8.4 & 7.2 & 691 & \\
\hline $140-93-34 d . .$. & Stark......... & 80 & June $24 .$. & ........ & 16 & .80 & 429 & 415 & 424 & \\
\hline $140-102-27 \ldots$ & Billings..... & 265 & & & 13 & .40 & 27 & 14 & 384 & \\
\hline $140-102-27 \ldots$ & ....do........ & 515 & .............. & ........ & 56 & 2.4 & 11 & 22 & 458 I. & \\
\hline $140-102-27 a .$. & ....do......... & 300 & July $20 .$. & (........ & 8.6 & .33 & 4.0 & 2.9 & 456 & \\
\hline
\end{tabular}


Cannonball member of the Fort Union formation, and upperpart of the Fort Union North Dakota

Survey. Analytical results in parts per million except as indicated]

\begin{tabular}{|c|c|c|c|c|c|c|c|c|c|c|c|c|c|}
\hline \multirow{2}{*}{$\begin{array}{c}\mathrm{Bi}- \\
\text { car- } \\
\text { bon- } \\
\text { ate } \\
\left(\mathrm{HCO}_{3}\right)\end{array}$} & \multirow{2}{*}{$\begin{array}{c}\mathrm{Car}- \\
\text { bon- } \\
\text { ate } \\
\left(\mathrm{CO}_{3}\right)\end{array}$} & \multirow{2}{*}{$\begin{array}{c}\text { Sulfate } \\
\left(\mathrm{SO}_{4}\right)\end{array}$} & \multirow[b]{2}{*}{$\begin{array}{l}\text { Chlo- } \\
\text { ride } \\
\text { (Cl) }\end{array}$} & \multirow[b]{2}{*}{$\begin{array}{l}\text { Fluso- } \\
\text { ride } \\
\text { (F) }\end{array}$} & \multirow[b]{2}{*}{$\begin{array}{l}\mathrm{Ni}- \\
\text { trace } \\
\left(\mathrm{NO}_{3}\right)\end{array}$} & \multirow[b]{2}{*}{$\begin{array}{l}\text { Bo- } \\
\text { ron } \\
\text { (B) }\end{array}$} & \multirow{2}{*}{$\begin{array}{l}\text { Dis- } \\
\text { solved } \\
\text { solids } \\
\text { (residue } \\
\text { on } \\
\text { evapo- } \\
\text { ration } \\
\text { at } \\
180^{\circ} \mathrm{C} \text { ) }\end{array}$} & \multicolumn{2}{|c|}{$\begin{array}{l}\text { Hardness } \\
\text { as } \\
\mathrm{CaCO}_{3}\end{array}$} & \multirow{2}{*}{$\begin{array}{l}\text { Per- } \\
\text { cent } \\
\text { sodi - } \\
\text { um }\end{array}$} & \multirow{2}{*}{$\begin{array}{c}\text { Specific } \\
\text { con- } \\
\text { duct- } \\
\text { ance } \\
\text { (micro- } \\
\text { mhos } \\
\text { at } \\
\left.25^{\circ} \mathrm{C}\right)\end{array}$} & \multirow{2}{*}{$\mathrm{pH}$} & \multirow{2}{*}{$\begin{array}{c}\text { Source } \\
\text { of } \\
\text { data }\end{array}$} \\
\hline & & & & & & & & $\begin{array}{c}\text { Cal- } \\
\text { cium, } \\
\text { mag- } \\
\text { nesium }\end{array}$ & $\begin{array}{l}\text { Non- } \\
\text { car- } \\
\text { bon- } \\
\text { ate }\end{array}$ & & & & \\
\hline 234 & ....... & 1,354 & 71 & 0.2 & 176 & & 2,546 & 1,170 & 978 & 37 & & & b \\
\hline 572 & ........ & 1,674 & 14 & .8 & 4.4 & $\ldots . .$. & 3,061 & 250 & 0 & 89 & ............. & & b \\
\hline 721 & ........ & 621 & 19 & .8 & 4.4 & ....... & 1,604 & 620 & 29 & 51 & & & b \\
\hline 426 & ........ & 794 & 50 & 0 & 176 & & 1,772 & 830 & 480 & 40 & $\ldots .$. & & $\mathrm{b}$ \\
\hline 669 & ........ & 524 & 4.4 & .2 & 4.4 & $\cdots \cdots$ & 1,352 & 843 & 295 & 24 & ............ & & b \\
\hline 289 & & 647 & 8.0 & 1.0 & 2.7 & & 1,231 & 420 & 84 & 55 & & & b \\
\hline 789 & & 384 & 7.5 & 1.6 & .08 & & 1,298 & 76 & 0 & 93 & ...... & & b \\
\hline 621 & & 252 & 22 & 1.6 & 3.1 & $\ldots .$. & 1,022 & 25 & 0 & 97 & ....... & & b \\
\hline 549 & ......... & 364 & 90 & .8 & 5.7 & ..... & 1,229 & 515 & 65 & 47 & & & b \\
\hline 610 & .......... & 573 & 5.0 & .2 & 1.3 & & 1,292 & 637 & 137 & 43 & .............. & & b \\
\hline 566 & 38 & 729 & 11 & ....... & 3.0 . & & 1,673 & 38 & 0 & 97 & & & a \\
\hline 493 & & 853 & 13 & .6 & 6.7 & & 1,759 & 45 & 0 & 97 & & & b \\
\hline 643 & ......... & 556 & 7.0 & 2.8 & 4.4 . & & 1,463 & 214 & 0 & 81 & ....... & & b \\
\hline 1,557 & ......... & 59 & 60 & 2.0 & 1.3 & & 1,603 & 53 & 0 & 96 & & & b \\
\hline 1,290 & & 15 & 23 & 3.2 & 3.5 . & & 1,227 & 24 & 0 & 98 & …................ & & b \\
\hline 590 & 34 & 400 & 6.0 & & 2.0 & & 1,208 & 37 & 0 & 96 & & & a \\
\hline 665 & & 378 & 6.0 & & 16 & & 1,236 & 90 & 0 & 91 & & & b \\
\hline 590 & & 289 & 60 & .8 & 1.3 & $\ldots \ldots$ & 1,019 & 197 & 0 & 78 & .............. & & $\mathrm{b}$ \\
\hline 607 & & 394 & 9.5 & .4 & 4.4 & ...... & 1,315 & 61 & 0 & 94 & .............. & & b \\
\hline 413 & 0 & 530 & 20 & .1 & .0 & & 1,230 & 629 & 290 & 32 & 1,540 & 7.3 & a \\
\hline 1,020 & 8 & 387 & 14 & .7 & 3.8 & & 1,530 & 29 & 0 & 97 & 2,270 & 8.3 & a \\
\hline 1,200 & 41 & 22 & 66 & ...... & 2.7 & & 1,272 & 23 & 0 & 98 & & & a \\
\hline 1,271 & 16 & 4.9 & 533 & ....... & ........ & & 2,059 & 76 & 0 & 96 & $\ldots$ & & a \\
\hline 1,220 & 0 & 11 & 208 & ...... & ........ & & 1,427 & 25 & 0 & 98 & ....... & & a \\
\hline 521 & ......... & 467 & 57 & .2 & 11 & & 1,203 & 512 & 83 & 49 & & & b \\
\hline 412 & ....... & 535 & 205 & .2 & 212 & & 1,671 & 1,087 & 750 & 15 & …......... & & $\mathrm{b}$ \\
\hline 1,195 & & 329 & 6.5 & 3.0 & 6.7 & & 1,600 & 83 & 0 & 94 & & & b \\
\hline 720 & & 323 & 5.0 & .2 & 2.2 & & 1,092 & 343 & 0 & 64 & & & b \\
\hline 449 & ........ & 445 & 11 & .4 & 53 & .... & 1,053 & 379 & 11 & 58 & & & b \\
\hline 689 & ......... & 524 & 6 & .8 & 3.1 & & 1,322 & 498 & 0 & 56 & ............ & $\cdots .$. & b \\
\hline 816 & 47 & 528 & 7.0 & .2 & .5 & 0.48 & 1,580 & 51 & 0 & 95 & 2,260 & 8.4 & a \\
\hline 671 & 0 & 1,020 & 12 & .2 & 1.2 : & & 2,060 & 510 & 0 & 68 & 2,670 & 7.1 & a \\
\hline 878 & 0 & 1,660 & 9.6 & .8 & .8 . & & 3,310 & 406 & 0 & 84 & 4,150 & 7.8 & a \\
\hline 1,250 & ......... & 44 & 13 & 6.5 & .9 & & 1,160 & 74 & 0 & 94 & ............. & $\cdots \cdots$ & b \\
\hline 1,274 & 0 & 537 & 4.0 & ........ & 2.7 & & 1,963 & 208 & 0 & 87 & & & a \\
\hline 847 & & 733 & 10 & 1.0 & 9.8 & & 1,950 & 104 & 0 & 93 & & & b \\
\hline 844 & .......... & 755 & 7.0 & 1.2 & 8.0 & & 1,969 & 120 & 0 & 92 & & & b \\
\hline 1,139 & 58 & 510 & 5.0 & ........ & 2.6 & & 1,874 & 51 & 0 & 97 & .............. & & a \\
\hline 593 & 0 & 2,994 & 8.0 & ........ & 2.8 & & 4,980 & 2,770 & 2,284 & 25 & & & a \\
\hline 501 & .......... & 506 & 11 & 1.6 & 4.4 . & …… & 1,290 & 125 & 0 & 87 & ............... & & b \\
\hline 863 & ......... & 290 & 14 & 1.6 & 6.7 & & 1,403 & 123 & 0 & 89 & & & b \\
\hline 522 & 38 & 506 & 8.0 & & & & 1,294 & 22 & 0 & 98 & & & a \\
\hline
\end{tabular}


Table 5.-Chemical analyses of saline ground water from the Hell Creek formation, formation, undifferentiated,

\begin{tabular}{|c|c|c|c|c|c|c|c|c|c|c|}
\hline Location & County & $\begin{array}{l}\text { Depth } \\
\text { of } \\
\text { well } \\
\text { (feet) }\end{array}$ & $\begin{array}{c}\text { Date } \\
\text { of } \\
\text { col- } \\
\text { lection }\end{array}$ & $\begin{array}{l}\text { Tem- } \\
\text { pera- } \\
\text { ture } \\
\left({ }^{\circ} \mathrm{F}\right)\end{array}$ & $\begin{array}{l}\text { Silica } \\
\left(\mathrm{SiO}_{2}\right)\end{array}$ & $\begin{array}{l}\text { Total } \\
\text { iron } \\
\text { (Fe) }\end{array}$ & $\begin{array}{l}\text { Cal- } \\
\text { cium } \\
\text { (Ca) }\end{array}$ & $\begin{array}{c}\text { Mag- } \\
\text { ne- } \\
\text { sium } \\
(\mathrm{Mg})\end{array}$ & $\begin{array}{l}\text { Sodium } \\
(\mathrm{Na})\end{array}$ & $\begin{array}{l}\text { Potas- } \\
\text { sium } \\
\text { (K) }\end{array}$ \\
\hline & & & $\underline{1921}$ & & & & & & & \\
\hline 140-102-27a.. & Billings..... & 505 & July $20 .$. & & 12 & 0.27 & 5.2 & 2.2 & \multicolumn{2}{|c|}{470} \\
\hline $140-104-29 \ldots$ & $\begin{array}{l}\text { Golden } \\
\text { Valley. }\end{array}$ & 186 & ............. & & 19 & 1.8 & 22 & 28 & 631 & \\
\hline $142-74-1 \ldots \ldots$ & Kidder...... & 160 & $\cdots \cdots$ & & 29 & .20 & 26 & 14 & 388 & \\
\hline $143-80-35 \ldots$. & McLean.... & 160 & & & 11 & .8 & 59 & 24 & 341 & $\ldots \ldots . .$. \\
\hline $143-83-6 b \ldots$. & Oliver....... & 130 & Jume 22 & ........ & 11 & .69 & 6.4 & 4.9 & 765 & \\
\hline $143-83-6 b \ldots$ & ...do........ & 200 & ....do.... & & 15 & .40 & 7.2 & 2.6 & 670 & \\
\hline $144-88-25$ & Mercer..... & 176 & .............. & & 14 & .1 & 13 & 16 & 596 & a....... \\
\hline $144-88-25 \mathrm{cb}$ & ....do......... & 63.3 & $\frac{1947}{\text { May } 13 . .}$ & & 11 & .20 & 12 & 5.9 & 504 & 7.2 \\
\hline $144-91-10$ & Dunn.......... & ......... & ............. & & 5.0 & 1.2 & 81 & 36 & 502 & ......... \\
\hline $145-92-25$ & ....do........ & 20 & .............. & & 28 & 0 & 85 & 61 & 219 & .......... \\
\hline $145-92-25 \ldots \ldots$ & ....do......... & 18 & $\ldots \ldots$ & & 14 & 1.4 & 78 & 92 & 344 & ......... \\
\hline $145-92-25 \ldots \ldots$ & ....do........ & 14 & $\cdots \cdots$ & & 18 & .40 & 138 & 100 & 156 & .......... \\
\hline $145-92-25 \ldots$. & ....do......... & 14 & & & 22 & .40 & 133 & 85 & 249 & ........... \\
\hline $145-92-25$ & ....do......... & 16 & $\ldots$. & & 24 & .50 & 132 & 96 & 111 & ........... \\
\hline $145-92-25 \ldots \ldots$ & ....do........ & 19 & ...... & ......... & 28 & 1.2 & 187 & 108 & 160 & ............ \\
\hline $145-92-25$. & .....do......... & 12 & $\cdots \cdots$ & ......... & 19 & .40 & 50 & 58 & 224 & ............ \\
\hline $145-92-25 \ldots$. & ....do......... & 11 & $\cdots \cdots$ & (....... & 35 & .90 & 70 & 48 & 485 & ............ \\
\hline $145-92-25 .$. & ....do......... & 18 & & ... & 33 & 1.3 & 92 & 79 & 360 & \\
\hline $145-92-25 \ldots .$. & ....do......... & 20 & $\ldots \ldots$ & ..... & 30 & .60 & 72 & 49 & 378 & ........... \\
\hline $145-92-25 \ldots$. & ....do......... & 18 & .............. & ........ & 31 & .8 & 70 & 59 & 376 & ........... \\
\hline $145-92-25 \ldots$. & ....do......... & 14 & .............. & ........ & 32 & .60 & 211 & 113 & 712 & ........... \\
\hline $145-92-25 \ldots$. & ....do......... & 20 & ............. & ........ & 20 & 2.4 & 70 & 75 & 605 & ........... \\
\hline $145-92-25 \ldots$. & ....do........ & 20 & & & 18 & .2 & 96 & 85 & 774 & ........... \\
\hline $145-92-25 \ldots$. & ....do......... & 20 & & ..... & 18 & .9 & 61 & 39 & 604 & ........... \\
\hline $145-92-25 \ldots$. & ....do........ & 110 & .......... & ....... & 74 & 2.6 & 25 & 13 & 680 & ........... \\
\hline $145-94-26 \ldots$ & ....do......... & $50-60$ & ..... & ..... & 22 & 4.0 & 22 & 25 & 312 & .......... \\
\hline $145-94-26 \ldots$. & ....do......... & 18 & .............. & & 6.0 & 8.0 & 167 & 132 & 130 & ........... \\
\hline $145-95-9 a \ldots$. & ....do......... & Spring & $\frac{1921}{\text { June } 22 . .}$ & & 18 & .14 & 16 & 15 & 34 & \\
\hline $146-77-11 \ldots$ & Sheridan... & 430 & ............. & & 22 & 1.2 & 50 & 14 & 447 & a........ \\
\hline $146-88-5 \mathrm{cab}$ & Mercer..... & 65 & $\frac{1950}{\text { Aug. } 9 . .}$ & & 28 & .26 & 84 & 40 & 340 & 7.6 \\
\hline $146-91-5 \mathrm{cbal}$ & Dunn........ & Spring & ....do.... & 48 & 15 & .20 & 11 & 8.3 & 744 & 4.8 \\
\hline $147-80-28 \ldots$. & McLean.... & $413^{\circ}$ & ........... & .......... & 16 & 0 & 13 & 23 & 618 & ...... \\
\hline $147-89-18 c d c$ & Mercer..... & 105 & Aug. 9 & & 18 & 8.1 & 77 & 53 & 260 & 7.8 \\
\hline $147-90-5$ aаa. & McLean.... & 620 & Aug. 10 & 49 & 12 & .16 & 6.0 & 1.2 & 914 & 3.3 \\
\hline $147-90-22 \mathrm{ccc}$ & Mercer..... & 150 & Nov. $3 .$. & 47 & 17 & 1.7 & 80 & 54 & 332 & 8.5 \\
\hline $147-90-25 a b c$ & ....do......... & 155 & ....do.... & & 13 & 1.5 & 26 & 18 & 928 & 5.6 \\
\hline $147-93-3 \mathrm{dbb}$ & Dunn........ & 223 & Oct. 19.. & $\ldots \ldots . .$. & 26 & .16 & 9.0 & 11 & 1,060 & 4.4 \\
\hline $147-94-4 d d a$. & ....do........ & Spring & Aug. 8.. & 45 & 28 & 21 & 206 & 95 & 244 & 7.6 \\
\hline $148-84-4 \ldots \ldots$ & McLean.... & 121 & ............. & $\ldots \ldots$ & 20 & 1.4 & 33 & 29 & 448 & \\
\hline $148-84-8 \ldots \ldots$ & ....do......... & 140 & ............ & $\cdots \cdots$ & 27 & 1.3 & 53 & 33 & 368 & ........... \\
\hline $148-84-8 .$. & ....do........ & 122 & & ......... & 30 & 2.0 & 34 & 29 & 359 & \\
\hline $148-84-8 c \ldots$. & ....do........ & 125 & $\frac{1921}{\text { June } 18 . .}$ & & 27 & 3.0 & 47 & 24 & 43 & \\
\hline $148-88-9$ add. & ....do........ & 58 & $\frac{1950}{\text { Aug. } 9 . .}$ & $\mid \cdots \cdots \cdot$ & 10 & .87 & 23 & 12 & 680 & 8.9 \\
\hline
\end{tabular}


Cannonball member of the Fort Union formation, and upper part of the Fort Union North Dakota-Continued

\begin{tabular}{|c|c|c|c|c|c|c|c|c|c|c|c|c|c|}
\hline \multirow{2}{*}{$\begin{array}{c}\mathrm{Bi}- \\
\text { car- } \\
\text { bon- } \\
\text { ate } \\
\left(\mathrm{HCO}_{3}\right)\end{array}$} & \multirow{2}{*}{$\begin{array}{l}\text { Car- } \\
\text { bon- } \\
\text { ate } \\
\left(\mathrm{CO}_{3}\right)\end{array}$} & \multirow[b]{2}{*}{$\begin{array}{l}\text { Sulfate } \\
\left(\mathrm{SO}_{4}\right)\end{array}$} & \multirow{2}{*}{$\begin{array}{l}\text { Chlo- } \\
\text { ride } \\
\text { (Cl) }\end{array}$} & \multirow{2}{*}{$\begin{array}{l}\text { Fluo- } \\
\text { ride } \\
\text { (F) }\end{array}$} & \multirow{2}{*}{$\begin{array}{l}\mathrm{Ni}- \\
\text { trate } \\
\left(\mathrm{NO}_{3}\right)\end{array}$} & \multirow{2}{*}{$\begin{array}{l}\text { Bo- } \\
\text { ron } \\
\text { (B) }\end{array}$} & \multirow{2}{*}{$\begin{array}{l}\text { Dis- } \\
\text { solved } \\
\text { solids } \\
\text { (residue } \\
\text { on } \\
\text { evapo- } \\
\text { ration } \\
\text { at } \\
180^{\circ} \mathrm{C} \text { ) }\end{array}$} & \multicolumn{2}{|c|}{$\begin{array}{l}\text { Hardness } \\
\text { as } \\
\mathrm{CaCO}_{3}\end{array}$} & \multirow{2}{*}{$\begin{array}{c}\text { Per- } \\
\text { cent } \\
\text { sodi - } \\
\text { um }\end{array}$} & \multirow{2}{*}{$\begin{array}{c}\text { Specific } \\
\text { con- } \\
\text { duct- } \\
\text { ance } \\
\text { (micro- } \\
\text { mhos } \\
\text { at } \\
25^{\circ} \mathrm{C} \text { ) }\end{array}$} & \multirow{2}{*}{$\mathrm{pH}$} & \multirow{2}{*}{$\begin{array}{c}\text { Source } \\
\text { of } \\
\text { data }\end{array}$} \\
\hline & & & & & & & & $\begin{array}{c}\text { Cal- } \\
\text { cium, } \\
\text { mag- } \\
\text { nesium }\end{array}$ & $\begin{array}{l}\text { Non- } \\
\text { car- } \\
\text { bon- } \\
\text { ate }\end{array}$ & & & & \\
\hline 810 & 34 & 294 & 16 & & 3.4 & & 1,308 & 22 & 0 & 98 & & & a \\
\hline 422 & ........... & 1,131 & 13 & 1.8 & .9 & & 2,070 & 175 & 0 & 89 & &. & b \\
\hline 722 & .......... & 334 & 18 & 0 & 6.7 & & 1,136 & 126 & 0 & 87 & & & b \\
\hline 841 & ......... & 279 & 4.0 & .4 & 8.0 & & 1,145 & 253 & 0 & 75 & ............. & & $\mathrm{b}$ \\
\hline 1,674 & 72 & 145 & 56 & .......... & 4.0 & & 1,925 & 36 & 0 & 98 & ............. & & a \\
\hline 1,444 & 82 & 11 & 112 & ........ & & & 1,610 & 29 & 0 & 98 & & & a \\
\hline 1,570 & .......... & 58 & 30 & 1.8 & 3.5 & & 1,520 & 99 & 0 & 93 & & & $\mathrm{~b}$ \\
\hline 1,020 & 79 & 190 & .0 & 1.1 & 1.8 & 0.23 & 1,330 & 54 & 0 & 94 & 2,050 & 8.7 & a \\
\hline 629 & & 879 & 6.0 & 0 & 6.7 & & 1,894 & 353 & 0 & 76 & ............. & ..... & $\mathrm{b}$ \\
\hline 476 & .......... & 306 & 80 & .2 & 155 & ..... & 1,269 & 469 & 79 & 51 & & $\ldots \ldots$ & $\mathrm{b}$ \\
\hline 523 & .......... & 828 & 13 & .2 & 18 & ...... & 1,713 & 535 & 107 & 57 & ............. & ...... & $\mathrm{b}$ \\
\hline 441 & ......... & 559 & 26 & 0 & 155 & ....., & 1,336 & 765 & 403 & 31 & ............... & $\cdots \cdots$ & b \\
\hline 400 & & 441 & 70 & 0 & 425 & & 1,550 & 683 & 355 & 44 & ....... & $\cdots \cdots$ & b \\
\hline 468 & & 408 & 24 & 0 & 159 & & 1,143 & 729 & 345 & 25 & ................ & $\ldots$. & b \\
\hline 487 & & 768 & 48 & .4 & 1.3 & $\ldots \ldots$ & 1,680 & 914 & 0 & 28 & ............... & $\ldots \ldots$ & b \\
\hline 578 & & 272 & 38 & 1.2 & 49 & ...... & 1,129 & 367 & 0 & 57 & ............. & $\ldots \ldots$ & b \\
\hline 760 & & 579 & 40 & 1.2 & 6.6 & $\ldots \ldots$ & 1,634 & 374 & 0 & 74 & ............... & $\cdots \cdots$ & b \\
\hline 807 & ........ & 619 & 20 & 1.2 & 18 & & 2,381 & 571 & 0 & 59 & & $\cdots \cdots$ & b \\
\hline 734 & ........ & 427 & 108 & 1.2 & 8.9 & ..... & 1,424 & 387 & 0 & 68 & $\ldots .$. & $\ldots$. & b \\
\hline 583 & ..... & 599 & 58 & 1.2 & 66 & ....... & 1,494 & 421 & 0 & 66 & ............... & ..... & b \\
\hline 522 & & 1,452 & 326 & .8 & 177 & ..... & 3,366 & 995 & 567 & 61 & ............... & $\cdots \cdots$ & $\mathrm{b}$ \\
\hline 827 & .......... & 968 & 9.8 & 1.2 & 133 & & 2,327 & 490 & 0 & 73 & .............. & $\cdots \cdot$. & b \\
\hline 837 & & 1,501 & 12 & 1.6 & 5.8 & ....... & 2,855 & 590 & 0 & 74 & & $\ldots .$. & b \\
\hline 790 & ........ & 925 & 7.1 & 2.0 & 2.2 & ....... & 2,012 & 315 & 0 & 81 & ...... & $\ldots \ldots$ & b \\
\hline 1,390 & ........... & 327 & 9.9 & 0 & 2.7 & $\ldots \ldots$ & 1,813 & 127 & 0 & 93 & .............. & ...... & b \\
\hline 628 & ........... & 306 & 4.5 & 1.0 & 4.4 & ........ & 1,084 & 166 & 0 & 81 & ............... & ..... & b \\
\hline 356 & ......... & 906 & 9.0 & .1 & 13 & ..... & 1,798 & 976 & 684 & 22 & ……...... & $\cdots \cdots$ & b \\
\hline 615 & 0 & 320 & 2.0 & ........ & .5 & & 1,017 & 102 & 0 & 88 & $\ldots$. & $\ldots .$. & a \\
\hline 846 & ......... & 120 & 232 & 1.2 & 5.3 & $\ldots \ldots$ & 1,290 & 185 & 0 & 84 & .............. & $\ldots .$. & b \\
\hline 804 & 0 & 435 & 3.0 & .4 & 3.2 & .20 & 1,370 & 374 & 0 & 66 & 1,900 & 7.5 & a \\
\hline 780 & 18 & 955 & 5.0 & .8 & 1.7 & .20 & 2,220 & 62 & 0 & 96 & 3,070 & 8.2 & a \\
\hline 1,183 & ....... & 35 & 328 & 2.0 & .2 & ....... & 1,567 & 125 & 0 & 91 & .............. & $\ldots .$. & $\mathrm{b}$ \\
\hline 608 & 0 & 440 & 4.0 & .4 & .7 & .10 & 1,160 & 410 & 0 & 57 & 1,650 & 7.5 & a \\
\hline 1,940 & 0 & 2.5 & 314 & .6 & .8 & .20 & 2,260 & 20 & 0 & 99 & 3,460 & 8.1 & a \\
\hline 493 & 0 & 698 & 5.0 & & 1.5 & .30 & 1,450 & 422 & 18 & 63 & 2,010 & 7.9 & a \\
\hline 1,200 & 14 & 1,150 & 17 & .6 & 2.2 & .00 & 2,780 & 139 & 0 & 93 & 3,900 & 8.2 & a \\
\hline 1,510 & 86 & 920 & 11 & 1.0 & 2.5 & .00 & 2,890 & 68 & 0 & 97 & 4,060 & 8.7 & a \\
\hline 756 & 0 & 760 & 2.0 & .2 & .9 & .10 & 1,800 & 905 & 285 & 37 & 2,250 & 7.2 & a \\
\hline 926 & ....... & 366 & 27 & .6 & 4.4 & $\ldots \ldots$ & 1,382 & 210 & 0 & 83 & .............. & … & $\mathrm{b}$ \\
\hline 708 & .......... & 446 & 28 & .4 & 2.2 & & 1,382 & 288 & 0 & 74 & & & $\mathrm{~b}$ \\
\hline 695 & ......... & 368 & 21 & .6 & 6.6 & & 1,253 & .243 & 0 & 79 & & & $\mathrm{~b}$ \\
\hline 922 & 0 & 368 & 10 & ....... & 1.1 & $\ldots$ & 1,386 & 216 & 0 & 81 & ............. & & $a$ \\
\hline 1,280 & 0 & 490 & 5.0 & .4 & 4.2 & .20 & 1,900 & 107 & 0 & 93 & 2,650 & 7.3 & $a$ \\
\hline
\end{tabular}

$4613970-58-3$ 
Table 5.-Chemical analyses of saline ground water from the Hell Creek formation, formation, undifferentiated,

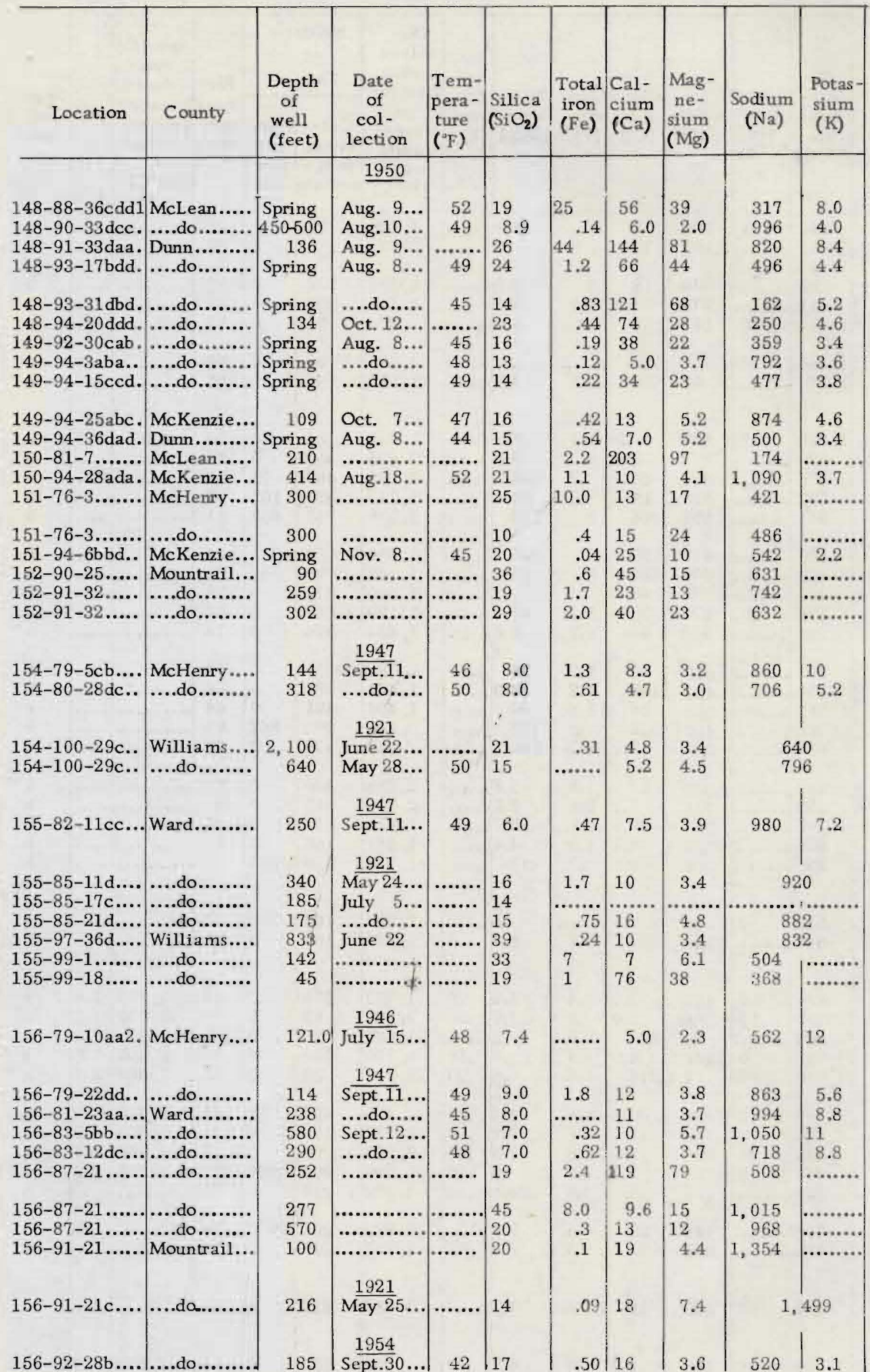


Cannonball member of the Fort Union formation, and upper part of the Fort Union North Dakota-Continued

\begin{tabular}{|c|c|c|c|c|c|c|c|c|c|c|c|c|c|}
\hline \multirow{2}{*}{$\begin{array}{c}\mathrm{Bi}- \\
\text { car- } \\
\text { bon- } \\
\text { ate } \\
\left(\mathrm{HCO}_{3}\right)\end{array}$} & \multirow{2}{*}{$\begin{array}{l}\mathrm{Car}- \\
\text { bon- } \\
\text { ate } \\
\left(\mathrm{CO}_{3}\right)\end{array}$} & \multirow{2}{*}{$\begin{array}{c}\text { Sulfate } \\
\left(\mathrm{SO}_{4}\right)\end{array}$} & \multirow{2}{*}{$\begin{array}{l}\text { Chlo- } \\
\text { ride } \\
\text { (C1) }\end{array}$} & \multirow{2}{*}{$\begin{array}{l}\text { Fluo- } \\
\text { ride } \\
\text { (F) }\end{array}$} & \multirow{2}{*}{$\begin{array}{l}\mathrm{Ni}- \\
\text { trate } \\
\left(\mathrm{NO}_{3}\right)\end{array}$} & \multirow{2}{*}{$\begin{array}{l}\text { Bo- } \\
\text { ron } \\
\text { (B) }\end{array}$} & \multirow{2}{*}{$\begin{array}{l}\text { Dis- } \\
\text { solved } \\
\text { solids } \\
\text { (residue } \\
\text { on } \\
\text { evapo- } \\
\text { ration } \\
\text { at } \\
180^{\circ} \mathrm{C} \text { ) }\end{array}$} & \multicolumn{2}{|c|}{$\begin{array}{l}\text { Hardness } \\
\text { as } \\
\mathrm{CaCO}_{3}\end{array}$} & \multirow{2}{*}{$\begin{array}{l}\text { Per- } \\
\text { cent } \\
\text { sodi- } \\
\text { um }\end{array}$} & \multirow{2}{*}{$\begin{array}{l}\text { Specific } \\
\text { con- } \\
\text { duct- } \\
\text { ance } \\
\text { (micro- } \\
\text { mhos } \\
\text { at } \\
25^{\circ} \mathrm{C} \text { ) }\end{array}$} & \multirow[b]{2}{*}{$\mathrm{pH}$} & \multirow{2}{*}{$\begin{array}{c}\text { Source } \\
\text { of } \\
\text { data }\end{array}$} \\
\hline & & & & & & & & $\begin{array}{c}\text { Cal- } \\
\text { cium, } \\
\text { mag- } \\
\text { nesium }\end{array}$ & $\begin{array}{l}\text { Non- } \\
\text { car- } \\
\text { bon- } \\
\text { ate }\end{array}$ & & & & \\
\hline 688 & 0 & 415 & 5.0 & 0.2 & 1.9 & 0.20 & 1,230 & 300 & 0 & 69 & 1,750 & 7.3 & a \\
\hline 2,420 & 0 & 2.5 & 149 & 1.4 & 1.1 & .20 & 2,400 & 23 & 0 & 99 & 3,540 & 8.1 & a \\
\hline 1,090 & 0 & 1,590 & 11 & .2 & 7.1 & .10 & 3,270 & 693 & 0 & 72 & 4,100 & 7.3 & a \\
\hline 862 & 0 & 700 & 2.0 & .6 & 1.7 & .20 & 1,820 & 346 & 0 & 75 & 2,500 & 7.7 & a \\
\hline 590 & 0 & 435 & 3.0 & .4 & 1.2 & .20 & 1,150 & 582 & 98 & 37 & 1,560 & 7.5 & a \\
\hline 581 & 0 & 378 & 2.0 & .6 & 1.5 & .10 & 1,050 & 300 & 0 & 64 & 1,490 & 8.0 & a \\
\hline 636 & 0 & 420 & 2,0 & .4 & 1.8 & .20 & 1,210 & 186 & 0 & 80 & 1,740 & 7.7 & a \\
\hline 972 & 59 & 790 & 3.0 & 1.4 & 5.2 & .20 & 2,230 & 28 & 0 & 98 & 3,100 & 8.3 & a \\
\hline 870 & 0 & 500 & 2.0 & .8 & 3.8 & .30 & 1,510 & 180 & 0 & 85 & 2,140 & 7.5 & a \\
\hline 964 & 18 & 1,110 & 4.0 & 1.0 & 2.8 & .30 & 2,540 & 54 & 0 & 97 & 3,440 & 8.3 & a \\
\hline 720 & 0 & 505 & 6.5 & 1.0 & 8.5 & & 1,490 & 39 & 0 & 96 & 2,090 & 7.7 & a \\
\hline 784 & ....... & 608 & 7.1 & .8 & 16 & & 1,579 & 921 & 278 & 29 & .............. & & b \\
\hline 1,440 & 47 & 1,140 & 4.5 & .8 & 2.7 & .20 & 3,070 & 42 & 0 & 98 & 4,140 & 8.5 & a \\
\hline 828 & ........ & 254 & 62 & .2 & 11 & ........ & 1,300 & 123 & 0 & 90 & & & b \\
\hline 1,020 & $\cdots$ & 248 & 66 & .2 & 6.7 & & 1,364 & 138 & 0 & 89 & & & b \\
\hline 1,010 & 0 & 450 & 8.0 & .6 & 1.7 & & 1,580 & 104 & 0 & 92 & 2,310 & 8.0 & a \\
\hline 1,218 & ........ & 519 & 5.0 & .2 & .3 & & 1,938 & 176 & 0 & 89 & & & b \\
\hline 686 & ........ & 736 & 8.6 & 1.0 & .9 & & 2,096 & 84 & 0 & 94 & $\ldots$. & & b \\
\hline 1,010 & .......... & 721 & 9.0 & .4 & .9 & ...... & 2,097 & 215 & 0 & 88 & & & b \\
\hline 908 & 0 & 4.8 & 825 & .3 & 2.5 & 2.4 & 2,260 & 34 & 0 & 99 & 3,880 & 7.7 & a \\
\hline 1,010 & 102 & 1.0 & 392 & .6 & 14 & 2.7 & 1,750 & 24 & 0 & 98 & 2,920 & 8.8 & a \\
\hline 1,332 & 0 & 6.6 & 236 & & ...... & & 1,580 & 26 & 0 & 98 & & & a \\
\hline 2,089 & 29 & 7.1 & 2.2 & $\ldots \ldots$ & .......... & $\cdots \cdots$ & 1,936 & 31 & 0 & 98 & ................ & $\cdots$ & a \\
\hline 1,030 & 0 & .8 & 940 & .5 & 1.2 & 2.4 & 2,500 & 34 & 0 & 98 & 4,340 & 7.6 & a \\
\hline 1, 954 & 0 & 2.6 & 294 & & & & 2,244 & 39 & 0 & 98 & & & a \\
\hline 1,920 & 55 & 30 & 106 & ....... & 1.3 & $\ldots . .$. & 2,300 & & 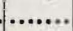 & ....... & & & a \\
\hline 2,152 & 29 & 31 & 110 & ........ & ..... & ...... & 2,345 & 60 & 0 & 97 & .............. & & a \\
\hline 1,479 & 34 & 8.7 & 422 & ......... & 1.9 & ..... & 2,124 & 39 & 0 & 98 & & $\ldots$ & a \\
\hline 664 & ........ & 576 & 7.4 & .8 & 3.1 & & 1,519 & 64 & 0 & 96 & & & b \\
\hline 666 & ......... & 564 & 15 & 1.2 & .09 & & 1,433 & 360 & 0 & 70 & .............. & $\cdots \cdots$ & b \\
\hline 936 & 22 & 7.5 & 340 & .3 & 2.8 & 4.6 & 1,460 & 22 & 0 & 97 & 2,380 & 8.0 & a \\
\hline 758 & 43 & 1.9 & 885 & .3 & 5.2 & 2.2 & 2,280 & 46 & 0 & 96 & 3,930 & 8.8 & a \\
\hline 720 & 0 & 1.6 & 1,150 & .3 & 1.5 & 1.9 & 2,610 & 42 & 0 & 98 & 4,540 & 8.2 & a \\
\hline 936 & 0 & 1.4 & 1,120 & .3 & 2.2 & 2.7 & 2,750 & 48 & 0 & 97 & 4,790 & 7.3 & a \\
\hline 1,280 & 0 & 137 & 295 & .3 & 10 & 1.3 & 1,870 & 45 & 0 & 97 & 2,950 & 8.2 & a \\
\hline 820 & ........ & 893 & 87 & 3 & .1 & $\ldots$. & 2,131 & 628 & 0 & 64 & & & b \\
\hline 1,065 & .......... & 1,351 & 19 & 1.6 & 2.7 & & 3,004 & 101 & 0 & 96 & & & b \\
\hline 2,030 & ........ & 1.5 & 354 & 3 & 1.8 & & 2,391 & 80 & 0 & 96 & & $\ldots$ & b \\
\hline 1,125 & ......... & 1,959 & 31 & 4 & .2 & $\ldots .$. & 3,971 & 35 & 0 & 98 & ................ & $\ldots \ldots$ & $\mathrm{b}$ \\
\hline 1,227 & 0 & 2,185 & 30 & ........ & 4.5 & ...... & 4,386 & 75 & 0 & 98 & ... & ...... & a \\
\hline 807 & 0 & 494 & 5.5 & .0 & 2.6 & & 1,440 & 55 & 0 & 95 & 2,170 & 8.1 & a \\
\hline
\end{tabular}


Table 5.-Chemical analyses of saline ground water from the Hell Creek formation, formation, undifferentiated,

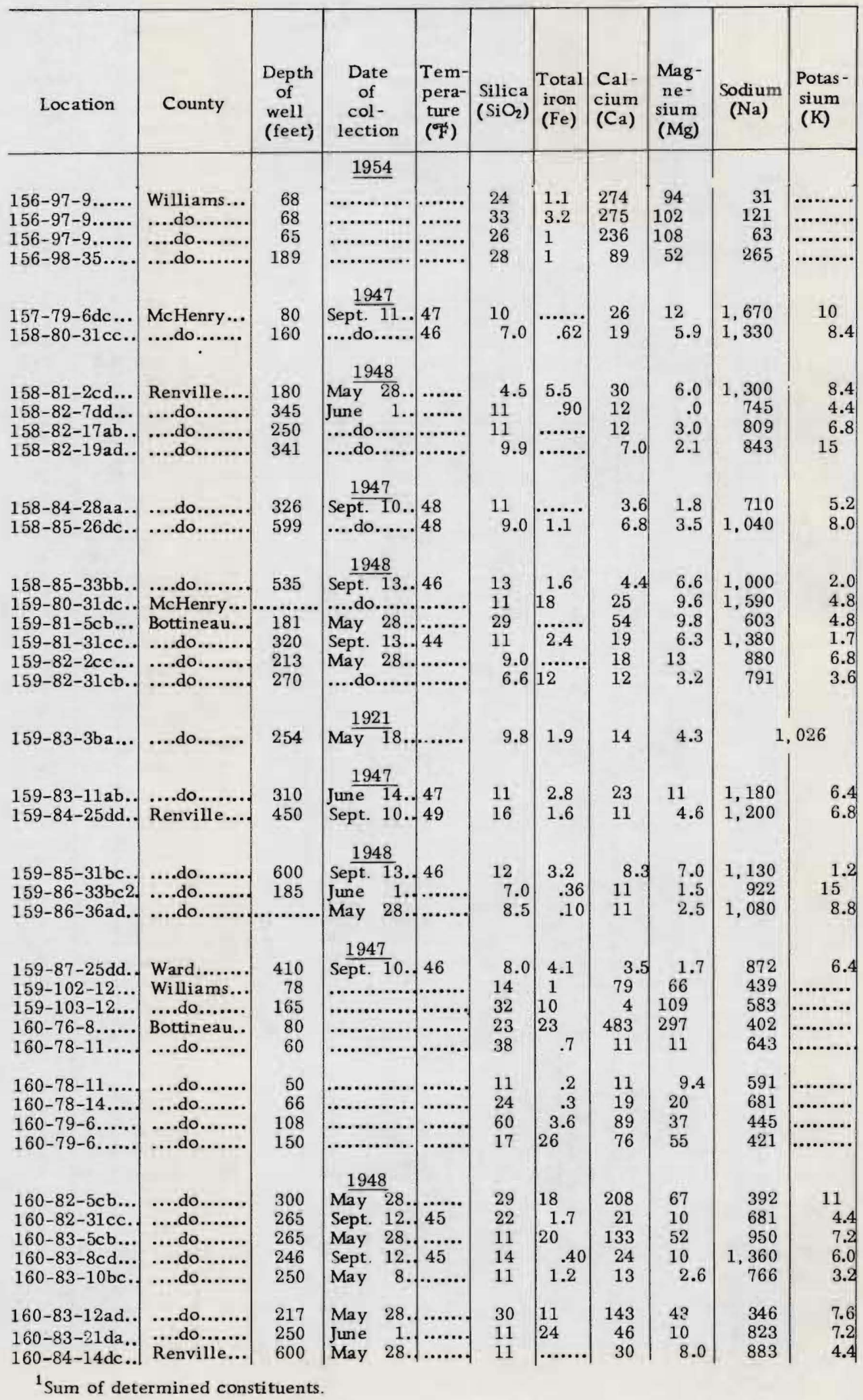


Cannonball member of the Fort Union formation, and upper part of the Fort Union North Dakota-Continued

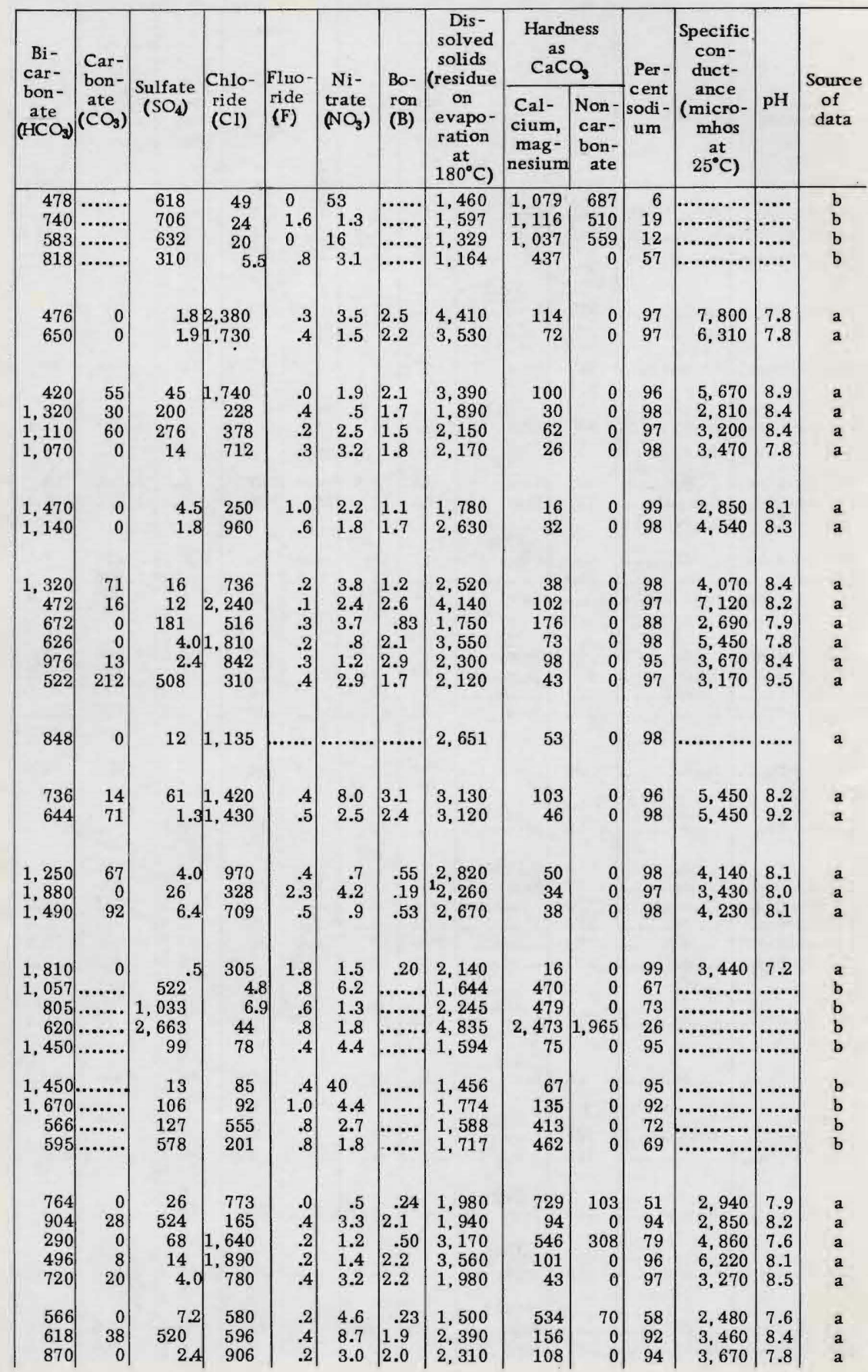


Table 5.-Chemical analyses of saline ground water from the Hell Creek formation, formation, undifferentiated,

\begin{tabular}{|c|c|c|c|c|c|c|c|c|c|c|}
\hline Location & County & $\begin{array}{l}\text { Depth } \\
\text { of } \\
\text { well } \\
\text { (feet) }\end{array}$ & $\begin{array}{l}\text { Date } \\
\text { of } \\
\text { col- } \\
\text { lection }\end{array}$ & $\begin{array}{l}\text { Tem- } \\
\text { pera- } \\
\text { ture } \\
\left({ }^{*} \mathrm{~F}\right)\end{array}$ & $\begin{array}{l}\text { Silica } \\
\left(\mathrm{SiO}_{2}\right)\end{array}$ & $\begin{array}{l}\text { Total } \\
\text { iron } \\
\text { (Fe) }\end{array}$ & $\begin{array}{l}\text { Cal- } \\
\text { cium } \\
(\mathrm{C} a)\end{array}$ & $\begin{array}{l}\text { Mag- } \\
\text { ne- } \\
\text { sium } \\
(\mathrm{Mg})\end{array}$ & $\begin{array}{l}\text { Sodium } \\
\text { (Na) }\end{array}$ & $\begin{array}{l}\text { Potas - } \\
\text { sium } \\
(\mathrm{K})\end{array}$ \\
\hline & & & 1947 & & & & & & & \\
\hline $\begin{array}{l}160-84-19 \mathrm{dd} . \\
160-87-24 \mathrm{bc} 1\end{array}$ & $\begin{array}{l}\text { Renville.... } \\
\text { Ward......... }\end{array}$ & $\begin{array}{l}400+ \\
280\end{array}$ & $\begin{array}{l}\text { Sept. } 10 . \\
\text { Sept. } 9 .\end{array}$ & $\begin{array}{l}49 \\
46\end{array}$ & $\begin{array}{l}26 \\
8.0\end{array}$ & $\begin{array}{c}3.0 \\
\ldots \ldots \ldots\end{array}$ & $\begin{array}{r}112 \\
3.2\end{array}$ & $\begin{array}{c}13 \\
3.0\end{array}$ & $\begin{array}{l}834 \\
682\end{array}$ & $\begin{array}{l}10 \\
14\end{array}$ \\
\hline $160-88-17 \ldots$ & ....do.......... & 400 & .................. & ......... & 19 & .6 & 21 & 16 & 527 & $\cdots \cdots \cdots$ \\
\hline $160-88-20 c \ldots$ & ....do.... & 313 & $\frac{1921}{\text { May } 19 .}$ & ......... & 32 & .58 & 30 & 16 & & 15 \\
\hline $161-82-20$ aa 1 & Bottineau... & 343 & $\frac{1947}{\text { Sept. } 8 .}$ & 47 & 11 & 1.2 & 83 & 21 & 2,730 & 19 \\
\hline 161-83-1aа... & ....do......... & 350 & Sept. 12. & 47 & 11 & 5.0 & 40 & 9.4 & 1,930 & .4 \\
\hline $161-83-18 c c$. & ....do...... & 340 & $\frac{1947}{\text { Sept. } 8 .}$ & 45 & 7.5 & 4.0 & 22 & 5.0 & 1,220 & 18 \\
\hline $161-84-3$ dd... & Renville. & 343 & $\begin{array}{c}\text { June } 15 . \\
1948\end{array}$ & 45 & 13 & 28 & 26 & 7.2 & 1,270 & 6.4 \\
\hline $161-84-4$ bb $1 .$. & ....do.. & 492 & Sept. 12 . & 47 & 10 & 4.0 & 76 & 20 & 2,420 & 1.6 \\
\hline $161-84-13 \ldots$. & ....do.......... & 343 & ................ & $\cdots \ldots .$. & 24 & .3 & 37 & 19 & 1,006 & ........... \\
\hline $161-84-16 \mathrm{dd}$. & ....do.......... & .......... & $\begin{array}{c}\text { Sept. } 12 . \\
1947\end{array}$ & 44 & 9.5 & 4.0 & 55 & 16 & 2,090 & 1.2 \\
\hline $\begin{array}{l}161-85-23 a b . \\
161-86-6 b c 2 .\end{array}$ & $\begin{array}{l}\text {....do... } \\
\text {....do.. }\end{array}$ & $\begin{array}{l}300 \\
400\end{array}$ & $\begin{array}{l}\text { Sept. } \\
\text { Sept. } \\
8 .\end{array}$ & $\begin{array}{l}46 \\
48\end{array}$ & $\begin{array}{l}9.6 \\
9.0\end{array}$ & $\begin{array}{l}2.1 \\
27\end{array}$ & $\begin{array}{l}26 \\
17\end{array}$ & 13 & $\begin{array}{r}1,000 \\
543\end{array}$ & ${ }^{18} .0$ \\
\hline $161-87-1$ bc... & ....do........... & 426 & ....do..... & 48 & 10 & $\ldots . .$. & 6.0 & 3.5 & 647 & 6.0 \\
\hline $161-87-17 b c 2$ & ....do.......... & 315 & Sept. 9. & 47 & 10 & 1.3 & 40 & 9.4 & 666 & ........... \\
\hline $161-89-3 \mathrm{dc} 1$ & Burke.. & 94 & $\frac{1948}{\text { Sept. } 11 .}$ & 44 & 19 & 1.0 & 82 & 29 & 905 & 4.0 \\
\hline $161-89-5 b \ldots$. & ....do... & 155 & May 20. & ........ & 12 & .10 & 16 & 6.6 & & 85 \\
\hline $161-89-5 \mathrm{dd} 1$. & ....do.. & 97 & $\frac{1948}{\text { Sept. } 11 .}$ & 44 & 12 & 2.0 & 12 & 5.2 & 605 & 1.6 \\
\hline $161-89-6$ da... & ....do.. & 70 & ....do...... & 44 & 12 & .20 & 7.5 & 4.2 & 565 & 2.0 \\
\hline 161-89-8cb... & ....do... & 102 & ....do...... & 44 & 9.4 & 8.0 & 89 & 44 & 577 & 2.4 \\
\hline 161-89-9bb2. & ....do.......... & 190 & ....do...... & 43 & 13 & 8.0 & 83 & 49 & 586 & 2.8 \\
\hline $161-89-21 \mathrm{da}$ & ....do.......... & 100 & ....do...... & 44 & 13 & .08 & 5.8 & 4.4 & 552 & 2.4 \\
\hline $161-89-26 \mathrm{dc}$. & .....do........ & 237 & $\frac{1947}{\text { June }} 6$. & 50 & 14 & .78 & 8.5 & 5.0 & 763 & 2.4 \\
\hline $161-90-11 \mathrm{~cd}$. & ....do........ & 89 & & ........ & 24 & 2.8 & 249 & 45 & 440 & 12 \\
\hline $162-75-30 b .$. & Bottineau... & 150 & May 17. & ......... & 21 & .10 & 4.4 & 1.5 & & 28 \\
\hline $162-75-30 c \ldots$ & ....do......... & 135 & May 16. & ........ & 31 & .13 & 6.0 & 1.7 & & 78 \\
\hline $162-84-21 \mathrm{cc}$ & Renville.... & 415 & $\frac{1947}{\text { Sept. }} 8$. & 48 & 8.0 & ........ & 65 & 18 & 2,300 & 14 \\
\hline $162-85-23 \mathrm{dd}$ & ....do........ & 500 & $\frac{1948}{\text { Sept. } 12 .}$ & 47 & 11 & 1.5 & 62 & 16 & 2,170 & .8 \\
\hline $\begin{array}{l}162-85-29 b b \\
162-88-36 c c\end{array}$ & $\begin{array}{l}\text {....do... } \\
\text { Burke.. }\end{array}$ & 308 & $\begin{array}{l}\frac{1947}{\text { Sept. } 8 .} \\
\text { June } 11 .\end{array}$ & $\begin{array}{l}45 \\
50\end{array}$ & $10^{6.0}$ & $\begin{array}{l}2.6 \\
6.0\end{array}$ & $\begin{array}{l}13 \\
7.0\end{array}$ & $\begin{array}{l}5.4 \\
4.2\end{array}$ & $\begin{array}{l}996 \\
460\end{array}$ & $\begin{array}{l}9.6 \\
7.2\end{array}$ \\
\hline
\end{tabular}


Cannonball member of the Fort Union formation, and upper part of the Fort Union North Dakota-Continued

\begin{tabular}{|c|c|c|c|c|c|c|c|c|c|c|c|c|c|}
\hline \multirow[b]{2}{*}{$\begin{array}{c}\mathrm{Bi}- \\
\text { car- } \\
\text { bon- } \\
\text { ate } \\
\left(\mathrm{HCO}_{3}\right)\end{array}$} & \multirow[b]{2}{*}{$\begin{array}{l}\text { Car- } \\
\text { bon- } \\
\text { ate } \\
\left(\mathrm{CO}_{3}\right)\end{array}$} & \multirow[b]{2}{*}{$\begin{array}{l}\text { Sulfate } \\
\left(\mathrm{SO}_{4}\right)\end{array}$} & \multirow[b]{2}{*}{$\begin{array}{l}\text { Chlo- } \\
\text { ride } \\
(\mathrm{Cl})\end{array}$} & \multirow[b]{2}{*}{$\begin{array}{l}\text { Fluo- } \\
\text { ride } \\
\text { (F) }\end{array}$} & \multirow[b]{2}{*}{$\begin{array}{c}\mathrm{Ni}- \\
\text { trate } \\
\left(\mathrm{NO}_{3}\right)\end{array}$} & \multirow{2}{*}{$\begin{array}{l}\text { Bo- } \\
\text { ron } \\
\text { (B) }\end{array}$} & \multirow{2}{*}{$\begin{array}{c}\text { Dis- } \\
\text { solved } \\
\text { solids } \\
\text { (resiclue } \\
\text { on } \\
\text { evapo- } \\
\text { ration } \\
\text { at } \\
180^{\circ} \mathrm{C} \text { ) } \\
\end{array}$} & \multicolumn{2}{|c|}{$\begin{array}{c}\text { Hardness } \\
\text { as } \\
\mathrm{CaCO}\end{array}$} & \multirow{2}{*}{$\begin{array}{l}\text { Per- } \\
\text { cent } \\
\text { sodi- } \\
\text { um }\end{array}$} & \multirow{2}{*}{$\begin{array}{c}\text { Specific } \\
\text { con- } \\
\text { duct- } \\
\text { ance } \\
\text { (micro- } \\
\text { mhos } \\
\text { at } \\
25^{\circ} \mathrm{C} \text { ) }\end{array}$} & \multirow{2}{*}{$\mathrm{pH}$} & \multirow[b]{2}{*}{$\begin{array}{c}\text { Source } \\
\text { of } \\
\text { data }\end{array}$} \\
\hline & & & & & & & & $\begin{array}{c}\text { Cal- } \\
\text { cium, } \\
\text { mag- } \\
\text { nesium }\end{array}$ & $\begin{array}{l}\text { Non- } \\
\text { car- } \\
\text { bon- } \\
\text { ate }\end{array}$ & & & & \\
\hline 906 & 0 & 246 & 820 & 0.5 & 1.8 & 1.6 & 2,530 & 333 & 0 & 84 & 4,210 & 8.1 & a \\
\hline 1,220 & 92 & 6.0 & 250 & 2.0 & 4.5 & .36 & 1,690 & 20 & 0 & 97 & 2,760 & 8.7 & a \\
\hline 1,180 & ........ & 95 & 137 & 1.6 & 4.4 & $\cdots \cdots \cdot$ & 1,380 & 117 & 0 & 91 & .............. & ...... & b \\
\hline 1,186 & 0 & 100 & 134 & ........ & 4.4 & $\cdots \cdots$ & 1,433 & 141 & 0 & 89 & ........ & $\cdots \cdots \cdot$ & a \\
\hline 257 & 0 & 25 & $4,290^{\circ}$ & .0 & 1.0 & 2.1 & 7,390 & 294 & 83 & 95 & 12,800 & 7.4 & a \\
\hline 378 & 0 & 4.2 & 2,900 & .0 & .5 & 1.7 & 5,080 & 138 & 0 & 97 & 8,760 & 7.9 & a \\
\hline 448 & 11 & 6.2 & 1,720 & .2 & 5.0 & 1.4 & 3,250 & 75 & 0 & 96 & 5,710 & 8.5 & a \\
\hline 468 & 35 & 4.9 & 1,780 & .2 & 1.0 & 1.8 & 3,410 & 94 & 0 & 96 & 6,050 & 8.5 & a \\
\hline 286 & 6 & 4.0 & 3,830 & .7 & .7 & 1.8 & 6,620 & 272 & 28 & 95 & 11,200 & 8.1 & $a$ \\
\hline 769 & ........ & 40 & 1,190 & 0 & 18 & ....... & 2,692 & 177 & 0 & 93 & ............... & $\ldots$ & b \\
\hline 304 & 16 & 4.8 & 3,200 & .2 & 1.5 & 1.8 & 5,560 & 203 & 0 & 96 & 8,380 & 8.3 & a \\
\hline 708 & 0 & 6.6 & 1,250 & .2 & 9.0 & .63 & 2,700 & 78 & 0 & 96 & 4,640 & 7.8 & a \\
\hline 914 & 37 & 271 & 138 & .6 & 2.2 & .50 & 1,510 & 96 & 0 & 92 & 2,320 & 8.7 & a \\
\hline 1,160 & 0 & .6 & 346 & .8 & 1.8 & .61 & 1,650 & 30 & 0 & 97 & 2,710 & 7.1 & a \\
\hline 648 & 19 & 845 & 85 & 1.2 & .7 & .25 & 2,000 & 138 & 0 & 91 & 2,860 & 8.6 & a \\
\hline 984 & 0 & 1,400 & 16 & .7 & 5.3 & .00 & 3,030 & 324 & 0 & 86 & 3,940 & 7.6 & a \\
\hline 1,530 & 0 & 11 & 36 & ....... & 2.0 & ...... & 1,472 & 67 & 0 & 95 & ............. & …... & a \\
\hline 1,340 & 31 & 168 & 25 & .8 & 1.8 & .11 & 1,580 & 51 & 0 & 96 & 2,060 & 8.2 & a \\
\hline 1,440 & 35 & 64 & 29 & .4 & 3.1 & .10 & 1,410 & 36 & 0 & 97 & 1,880 & 8.3 & a \\
\hline 582 & 10 & 1,080 & 28 & .3 & 2.5 & .18 & 2,180 & 403 & 0 & 76 & 2,570 & 8.1 & a \\
\hline 848 & 0 & 920 & 14 & 1.0 & 4.0 & .09 & 2,260 & 409 & 0 & 76 & 2,870 & 7.8 & a \\
\hline 1,400 & 24 & 4.0 & 50 & .4 & 2.1 & .11 & 1,400 & 33 & 0 & 97 & 1,920 & 8.2 & a \\
\hline 964 & 24 & 814 & 36 & 1.4 & 1.8 & .27 & 2,170 & 42 & 0 & 97 & 3,090 & 8.3 & a \\
\hline 650 & 0 & 1,120 & 18 & .2 & 2.4 & .42 & 2,270 & 806 & 273 & 54 & 2,640 & 7.1 & a \\
\hline 644 & 31 & 150 & 166 & ........ & 3.8 & ....... & 1,159 & 17 & 0 & 98 & ................ & $\cdots \cdots$ & a \\
\hline 764 & 12 & 10 & 306 & ........ & ......... & ...... & 1,269 & 22 & 0 & 98 & .............. & ....... & a \\
\hline 261 & 34 & 1.5 & 3,520 & .0 & 2.2 & 2.1 & 6,220 & 236 & 0 & 95 & 10,700 & 8.2 & $a$ \\
\hline 350 & 0 & .8 & 3,400 & .2 & .8 & 1.9 & 5,860 & 220 & 0 & 96 & 9,970 & 7.8 & a \\
\hline 723 & 0 & $1 \mathrm{~A}$ & 1,160 & .3 & 2.2 & 1.2 & 2,600 & 54 & 0 & 97 & 4,620 & 7.4 & a \\
\hline 864 & 63 & 112 & 95 & 1.6 & .5 & .51 & 1,290 & 35 & 0 & 96 & 2,080 & 8.6 & a \\
\hline
\end{tabular}


Table 5.-Chemical analyses of saline ground water from the $\mathrm{Hell}$ Creek formation, formation, undifferentiated,

\begin{tabular}{|c|c|c|c|c|c|c|c|c|c|c|}
\hline Location & County & $\begin{array}{l}\text { Depth } \\
\text { of } \\
\text { well } \\
\text { (feet) }\end{array}$ & $\begin{array}{l}\text { Date } \\
\text { of } \\
\text { col- } \\
\text { lection }\end{array}$ & $\begin{array}{l}\text { Tem- } \\
\text { pera- } \\
\text { ture } \\
\left({ }^{\circ} \mathrm{F}\right)\end{array}$ & $\begin{array}{l}\text { Silica } \\
\left(\mathrm{SiO}_{2}\right)\end{array}$ & $\begin{array}{c}\text { Total } \\
\text { iron } \\
(\mathrm{Fe})\end{array}$ & $\begin{array}{l}\text { Cal- } \\
\text { cium } \\
(\mathrm{Ca})\end{array}$ & $\begin{array}{l}\text { Mag- } \\
\text { ne-- } \\
\text { sium } \\
(\mathrm{Mg})\end{array}$ & $\begin{array}{l}\text { Sodium } \\
\text { (Na) }\end{array}$ & $\begin{array}{l}\text { Potas- } \\
\text { sium } \\
\text { (K) }\end{array}$ \\
\hline $\begin{array}{l}162-89-18 \text { ad.. } \\
162-89-31 \text { da.. } \\
162-91-3 d d 2 . . \\
162-91-27 \text { ac.. } \\
162-93-9 \text { ab1.. }\end{array}$ & $\begin{array}{l}\text { Burke......... } \\
\text { …do......... } \\
\text {....do......... } \\
\text { …do......... } \\
\text {....do......... }\end{array}$ & $\begin{array}{l}200 \\
270 \\
360 \\
106 \\
100\end{array}$ & \begin{tabular}{lr}
\multicolumn{2}{c}{1948} \\
May & 29 \\
Sept. & 11 \\
June & 2 \\
Sept. & 11 \\
....do.....
\end{tabular} & $\begin{array}{r}\cdots \ldots . . . \\
47 \\
\cdots \ldots \ldots \\
45 \\
45\end{array}$ & $\begin{array}{l}3.5 \\
11 \\
6.5 \\
12 \\
17\end{array}$ & $\begin{array}{c}\ldots . . . . \\
0.12 \\
3.2 \\
.32 \\
.80\end{array}$ & $\begin{array}{c}28 \\
7.5 \\
7.0 \\
9.0 \\
4.4\end{array}$ & $\begin{array}{r}15 \\
4.8 \\
1.3 \\
4.8 \\
4.4\end{array}$ & $\begin{array}{l}508 \\
752 \\
548 \\
712 \\
698\end{array}$ & $\begin{array}{l}11 \\
2.0 \\
18 \\
14 \\
.8\end{array}$ \\
\hline $\begin{array}{l}162-93-15 \text { bd } 1 . \\
162-95-4 \ldots \ldots . \\
162-95-4 \text { ac.... }\end{array}$ & $\begin{array}{l}\text {....do........... } \\
\text { Divide........ } \\
\text {....do........ }\end{array}$ & $\begin{array}{r}80 \\
365 \\
265\end{array}$ & $\begin{array}{l}\ldots . . \text { do..... } \\
\text { Ma......... }\end{array}$ & $\mid \begin{array}{l}\cdots \ldots . . \\
\cdots \cdots \\
\cdots \cdots \cdot . \cdot \\
\cdots\end{array}$ & \begin{tabular}{|l}
18 \\
8 \\
8.7
\end{tabular} & $\begin{array}{l}.50 \\
0 \\
\cdots \ldots . . .\end{array}$ & $\begin{array}{c}4.4 \\
12 \\
2.0\end{array}$ & $\begin{array}{l}6.6 \\
3.9 \\
2.1\end{array}$ & $\begin{array}{l}834 \\
786 \\
787\end{array}$ & $\begin{array}{r}2.0 \\
\ldots \ldots \ldots \\
1.2\end{array}$ \\
\hline $\begin{array}{l}162-96-5 \mathrm{dc} 1 . . \\
163-79-33 \ldots . . \\
163-80-34 \ldots .\end{array}$ & $\begin{array}{l}\text {....do.......... } \\
\text { Bottineau... } \\
\text {....do......... }\end{array}$ & $\begin{array}{r}320 \\
65 \\
140\end{array}$ & $\begin{array}{l}\text { June }^{\frac{1947}{4}} \\
\ldots \ldots \ldots \ldots . . . . .\end{array}$ & $\begin{array}{r}47 \\
\ldots \ldots . . \\
\cdots \ldots . .\end{array}$ & $\begin{array}{l}11 \\
14 \\
21\end{array}$ & $\begin{array}{r}3.5 \\
.4 \\
4.8\end{array}$ & $\begin{array}{l}14 \\
80 \\
44\end{array}$ & $\begin{array}{l}3.3 \\
77^{2} \\
18\end{array}$ & $\begin{array}{l}911 \\
419 \\
495\end{array}$ & $\begin{array}{c}13 \\
\ldots \ldots \ldots . . \\
\ldots \ldots \ldots . .\end{array}$ \\
\hline $163-84-30 c c$. & Renville.... & 325 & May 28 & ........ & 22 & 6.0 & 88 & 29 & 724 & 3.6 \\
\hline $\begin{array}{l}163-85-10 \mathrm{ba} . . \\
163-85-29 \mathrm{~cd} . . \\
163-86-17 \mathrm{bb} 1 . \\
163-88-28 \mathrm{dd} . \\
163-90-32 . . .\end{array}$ & $\begin{array}{l}\text {....do.......... } \\
\text {....do......... } \\
\ldots \ldots \text {............ } \\
\text { Burke.......... } \\
\text {....do......... }\end{array}$ & $\begin{array}{l}375 \\
500+ \\
716 \\
200 \\
223\end{array}$ & $\begin{array}{lr}\text { June } & 14 \\
\text { Sept. } & 8 \\
\text { June } & 6 \\
\text { June } & 5 \\
\text {............. }\end{array}$ & $\begin{array}{r}47 \\
48 \\
54 \\
50 \\
\ldots \ldots . . .\end{array}$ & $\begin{array}{l}11 \\
4.0 \\
7.6 \\
11 \\
27\end{array}$ & $\begin{array}{r}\cdots \ldots . . \\
1.6 \\
\cdots \ldots \ldots . \\
\cdots \ldots . . \\
1.4\end{array}$ & $\begin{array}{r}37 \\
54 \\
24 \\
18 \\
132\end{array}$ & $\begin{array}{l}10 \\
12 \\
7.0 \\
3.3 \\
54\end{array}$ & $\begin{array}{r}1,530 \\
2,150 \\
1,400 \\
802 \\
373\end{array}$ & $\begin{array}{c}8.0 \\
12 \\
11 \\
7.2 \\
\cdots . . . . .\end{array}$ \\
\hline $\begin{array}{l}\text { 163-93-8ad1.. } \\
163-93-29 \text { dd.. } \\
163-93-32 \text { bd.. }\end{array}$ & 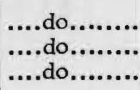 & $\begin{array}{l}204 \\
82.0 \\
270\end{array}$ & $\begin{array}{l}\text { June } \quad 4 \\
\ldots \ldots \text { do.... } \\
\ldots \ldots . . . . . .\end{array}$ & $\begin{array}{r}46 \\
48 \\
\ldots \ldots\end{array}$ & $\begin{array}{l}8.0 \\
27 \\
10\end{array}$ & $\begin{array}{l}.40 \\
.90 \\
.15\end{array}$ & $\begin{array}{c}8.0 \\
89 \\
.71\end{array}$ & $\begin{array}{c}4.4 \\
43 \\
4.4\end{array}$ & $\begin{array}{l}693 \\
519 \\
859\end{array}$ & $\begin{array}{l}7.2 \\
10^{7} \\
\ldots \ldots . .\end{array}$ \\
\hline $163-94-6 \mathrm{cb} . .$. & ....do......... & 260 & $\frac{1948}{\text { June }} 2$ & ........ & 5.5 & .03 & 29 & 11 & 711 & 7.6 \\
\hline $\begin{array}{l}163-94-33 \mathrm{aa} 1 . \\
163-96-13 \mathrm{da} . .\end{array}$ & ....do.......... & $\begin{array}{l}270 \\
105\end{array}$ & $\begin{array}{l}\text { June }_{\text {....do..... }}^{1947} \\
\text {... }\end{array}$ & $\begin{array}{l}50 \\
50\end{array}$ & 11 & $\begin{array}{l}3.0 \\
5.0\end{array}$ & $\begin{array}{l}26 \\
59\end{array}$ & $38^{6.1}$ & $\begin{array}{l}76 \overrightarrow{5} \\
729\end{array}$ & $\begin{array}{l}41 \\
11\end{array}$ \\
\hline $163-97-6 \mathrm{cc} 1 .$. & ....do.......... & 264 & $\frac{1948}{\text { Sept. } 12}$ & 47 & 13 & .80 & 3.5 & 4.8 & 698 & .8 \\
\hline $163-97-6 d \ldots . .$. & ....do.......... & 253 & May 21 & ....... & 33 & .27 & 22 & 8.0 & & 17 \\
\hline $\begin{array}{l}163-97-6 d c . . . \\
163-97-29 . . .\end{array}$ & …do........... & $\begin{array}{l}242 \\
775\end{array}$ & $\frac{1947}{\text { May } 2}$ & …... & $\begin{array}{l}30 \\
18\end{array}$ & .05 & $\begin{array}{c}20 \\
6.4\end{array}$ & $14^{7.6}$ & $\begin{array}{l}609 \\
954\end{array}$ & .0 \\
\hline $163-97-29 d \ldots$ & ....do.......... & 407 & $\frac{1921}{\text { May } 21}$ & ........ & 21 & .07 & 5.6 & 3.6 & & 700 \\
\hline $\begin{array}{l}163-97-31 b c . . \\
163-98-17 a a . .\end{array}$ & ....do............................ & $\begin{array}{l}250 \\
216\end{array}$ & $\begin{array}{l}\text { June } \\
\text { May }\end{array}$ & 50 & $\begin{array}{l}9.1 \\
7.6\end{array}$ & 9.0 & $\begin{array}{l}10 \\
10\end{array}$ & $\begin{array}{l}3.1 \\
2.0\end{array}$ & $\begin{array}{l}796 \\
586\end{array}$ & $\begin{array}{l}6.0 \\
9.6\end{array}$ \\
\hline
\end{tabular}


Cannonball member of the Fort Union formation, and upper part of the Fort Union North Dakota-Continued

\begin{tabular}{|c|c|c|c|c|c|c|c|c|c|c|c|c|c|}
\hline \multirow{2}{*}{$\begin{array}{c}\mathrm{Bi}^{-} \\
\mathrm{car}^{-} \\
\text {bon- } \\
\text { ate } \\
\left(\mathrm{HCO}_{3}\right)\end{array}$} & \multirow{2}{*}{$\begin{array}{l}\text { Car- } \\
\text { bon- } \\
\text { ate } \\
\left(\mathrm{CO}_{3}\right)\end{array}$} & \multirow{2}{*}{$\begin{array}{l}\text { Sulfate } \\
\left(\mathrm{SO}_{4}\right)\end{array}$} & \multirow{2}{*}{$\begin{array}{c}\text { Chlo- } \\
\text { ride } \\
(\mathrm{Cl})\end{array}$} & \multirow{2}{*}{$\begin{array}{l}\text { Fluo- } \\
\text { ride } \\
\text { (F) }\end{array}$} & \multirow{2}{*}{$\begin{array}{c}\mathrm{Ni}- \\
\text { trate } \\
\left(\mathrm{NO}_{3}\right)\end{array}$} & \multirow{2}{*}{$\begin{array}{l}\text { Bo- } \\
\text { ron } \\
\text { (B) }\end{array}$} & \multirow{2}{*}{$\begin{array}{c}\text { Dis- } \\
\text { solved } \\
\text { solids } \\
\text { (residue } \\
\text { on } \\
\text { evapo- } \\
\text { ration } \\
\text { at } \\
180^{\circ} \mathrm{C} \text { ) }\end{array}$} & \multicolumn{2}{|c|}{$\begin{array}{c}\text { Hardness } \\
\text { as } \\
\mathrm{CaCOs}\end{array}$} & \multirow{2}{*}{$\begin{array}{c}\text { Per- } \\
\text { cent } \\
\text { sodi- } \\
\text { um }\end{array}$} & \multirow{2}{*}{$\begin{array}{l}\text { Specific } \\
\text { con- } \\
\text { duct- } \\
\text { ance } \\
\text { (micro- } \\
\text { mhos } \\
\text { at } \\
25^{\circ} \mathrm{C} \text { ) }\end{array}$} & \multirow[b]{2}{*}{$\mathrm{pH}$} & \multirow{2}{*}{$\begin{array}{c}\text { Source } \\
\text { of } \\
\text { data }\end{array}$} \\
\hline & & & & & & & & $\begin{array}{c}\text { Cal- } \\
\text { cium, } \\
\text { mag- } \\
\text { nesium }\end{array}$ & $\begin{array}{c}\text { Non- } \\
\text { car- } \\
\text { bon- } \\
\text { ate }\end{array}$ & & & & \\
\hline 202 & 0 & 1,000 & 6.0 & 0.7 & 0.0 & 0.38 & 1,710 & 132 & 0 & 88 & 2,360 & 7.7 & a \\
\hline 1,680 & 0 & 6.4 & 186 & 1.4 & .0 & .17 & 1,810 & 38 & 0 & 98 & 2,530 & 7.9 & $\mathrm{a}$ \\
\hline 922 & 18 & 438 & 24 & 1.8 & 1.5 & .19 & 1,610 & 23 & 0 & 96 & 2,340 & 8.2 & a \\
\hline 1,850 & 0 & 4.0 & 40 & 1.0 & .5 & .10 & 1,730 & 42 & 0 & 96 & 2,320 & 8.0 & a \\
\hline 1,620 & 87 & 12 & 62 & 1.4 & .7 & .07 & 1,730 & 29 & 0 & 98 & 2,570 & 8.7 & a \\
\hline 1,900 & 87 & 68 & 55 & 1.2 & 2.1 & .15 & 2,070 & 38 & 0 & 98 & 2,970 & 8.4 & $\mathrm{a}$ \\
\hline 1,945 & ....... & 36 & 83 & 3.6 & 6.2 & $\ldots .$. & 1,956 & 52 & 0 & 97 & .............. & ..... & b \\
\hline 1,910 & 0 & .0 & 90 & 4.0 & 1.2 & .16 & 1,900 & 14 & 0 & 99 & 2,810 & 8.0 & a \\
\hline 1,230 & 108 & 784 & 44 & 2.0 & 2.5 & .59 & 2,520 & 48 & 0 & 97 & 3,730 & 8.8 & $a$ \\
\hline 410 & ........ & 1,019 & 19 & 1.8 & .09 & $\ldots \ldots$ & 1,946 & 518 & 182 & 64 & ............ & ....... & b \\
\hline 690 & ......... & - 332 & 248 & 1.2 & 3.5 & ...... & 1,588 & 191 & 0 & 85 & ............ & ....... & b \\
\hline 468 & 0 & 98 & 1,020 & .4 & 14 & .65 & 2,220 & 339 & 0 & 82 & 3,700 & 7.5 & a \\
\hline 356 & 14 & 9.9 & 2,360 & .3 & 1.0 & 1.6 & 4,170 & 133 & 0 & 96 & 7,590 & 8.4 & $a$ \\
\hline 310 & 0 & .9 & 3,320 & .4 & 1.0 & 2.3 & 5,800 & 184 & 0 & 95 & 10,400 & 6.7 & $a$ \\
\hline 730 & 24 & 725 & 1,250 & .6 & .2 & 1.9 & 3,880 & 89 & 0 & 97 & 6,490 & 8.4 & a \\
\hline 1,090 & 44 & 758 & 38 & 1.2 & 1.5 & .52 & 2,260 & 58 & 0 & 96 & 3,390 & 9.0 & a \\
\hline 633 & ....... & 776 & 21 & 1.6 & 8.0 & ...... & 1,728 & 592 & 73 & 60 & .............. & $\ldots .$. & b \\
\hline 1,420 & 45 & 166 & 70 & 2.4 & 1.5 & .48 & 1,740 & 38 & 0 & 97 & 2,690 & 8.2 & a \\
\hline 882 & 31 & 653 & 60 & .4 & 2.5 & .46 & 1,860 & 399 & 0 & 73 & 2,630 & 8.3 & a \\
\hline 1,927 & ...... & .41 & 212 & 3.6 & 1.3 & ...... & 2,110 & 20 & 0 & 99 & n........... & & b \\
\hline 518 & 10 & 1,140 & 28 & 1.2 & 1.4 & .16 & 2,250 & 118 & 0 & 92 & 3,020 & 8.3 & a \\
\hline 1,500 & 175 & 2.9 & 133 & 4.0 & .2 & .41 & 1,880 & 90 & 0 & 92 & 3,040 & & a \\
\hline 712 & 71 & 1,080 & 48 & 1.6 & 1.0 & .46 & 2,430 & 303 & 0 & 83 & 3,420 & 8.6 & a \\
\hline 1,450 & 90 & 90 & 100 & 1.8 & 3.6 & .85 & 1,730 & 28 & 0 & 98 & 2,590 & 8.5 & a \\
\hline 1,464 & 0 & 85 & 100 & ....... & 3.8 & ....... & 1,620 & 88 & 0 & 94 & (n.......... & & a \\
\hline 1,380 & 24 & 99 & 99 & .8 & 3.2 & .57 & 1,600 & 81 & 0 & 94 & 2,400 & 8.7 & a \\
\hline 1,375 & ....... & 72 & 668 & 1.4 & 2.7 & ...... & 2,457 & 75 & 0 & 97 & ............. & ....... & b \\
\hline 1,405 & 0 & 52 & 274 & $\ldots \ldots$ & .......... & ...... & 1,721 & 29 & 0 & 98 & (n.............. & & a \\
\hline 1,490 & 108 & 2.9 & 258 & 1.2 & 1.0 & 1.2 & 1,960 & 38 & 0 & 97 & 3,240 & & a \\
\hline 652 & 130 & 518 & 16 & .4 & 7.5 & .51 & 1,620 & 333 & 0 & 97 & 2,550 & 9.2 & a \\
\hline
\end{tabular}


The maximum dissolved-solids concentration was $7,390 \mathrm{ppm}$, and about 90 percent of the analyses showed dissolved-solids concentrations between 1,000 and 3,000 ppm. Most of the saline water that is known to be from the Hell Creek Formation and upper part of the Fort Union formation is of the sodium bicarbonate sulfate type; saline water from the Cannonball member is generally of the sodium chloride type. (See fig. 4.)

Because most of the saline water from these formations has a high percent sodium and a high boron content, it is unsuitable for irrigation. Water from many of the wells in these ayuifers has excessive color and hardness, and high concentrations of fluoride, sulfate, and iron.

\section{GLACIAL DRIFT AND ALLUVIUM}

Glacial drift of Pleistocene age covers approximately threefifths of North Dakota. In the unglaciated areas of the State, river alluvium of Recent age is widespread in the valleys of the Missouri River and its tributaries.

In areas covered with till, where little or no sorted material capable of yielding water is near the surface, lenses of sand and gravel in and at the base of the drift yield small supplies. However, because many of these lenses are completely drift covered, there is little recharge and they may be easily dewatered. Wells tapping sand and gravel in or at the base of the drift generally do not yield much more than $50 \mathrm{gpm}$. Wells in areas of surficial glacial outwash, however, yield small to large supplies of water, and some relatively widespread bodies of outwash buried in the drift yield similar supplies.

The hydrologic and lithologic characteristics of the drift and alluvial deposits are extremely variable both laterally and vertically; thus, the quantity and quality of the ground water that may be obtained from these surficial deposits also vary greatly.

Chemical analyses of saline water from the drift and alluvium are given in table 6 , and the chemical characteristics of the water are shown in figure 5. The patterns illustrate the many variations in type of water and degree of salinity.

Most of the data in table 6 represent water that is only slightly saline. However, water from wells 143-61-30cda2, 153-67-15cab, $162-90-10 \mathrm{cbc}$, and $163-84-6 \mathrm{ad}$ is very saline and contains large amounts of magnesium sulfate and sodium sulfate. 
Analyses of samples from wells 137-60-25aca, 142-50-2a, 143-88-4cd2, and 164-53-31ccb show the composition of several different types of water, in which calcium or sodium is the predominant cation and bicarbonate, sulfate, or chloride is the predominant anion. The waters are slightly to moderately saline. 
Table 6.-Chemical analyses of saline ground water

[Source of data: a, U. S. Geological Survey; b, North Dakota Geological Survey; c, North per million except

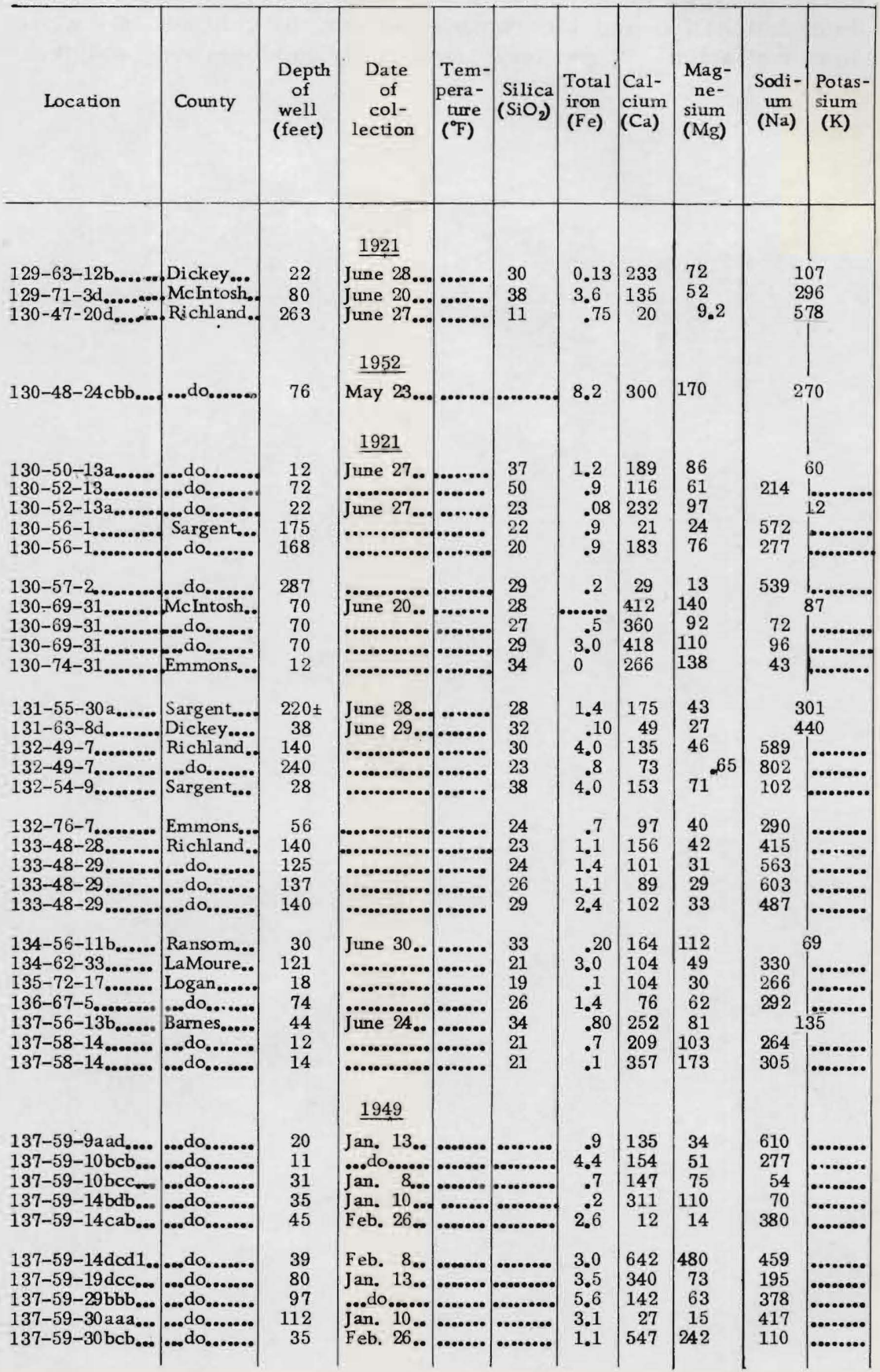


from glacial drift and alluvium, North Dakota

Dakota State Health Department or State Laboratories Department. Analytical results in parts as indicated]

\begin{tabular}{|c|c|c|c|c|c|c|c|c|c|c|c|c|c|}
\hline \multirow{2}{*}{$\begin{array}{c}\mathrm{Bi}- \\
\text { car- } \\
\text { bon- } \\
\text { ate } \\
\left(\mathrm{HCO}_{3}\right)\end{array}$} & \multirow{2}{*}{$\begin{array}{c}\text { Car- } \\
\text { bon- } \\
\text { ate } \\
\left(\mathrm{CO}_{3}\right)\end{array}$} & \multirow{2}{*}{$\begin{array}{c}\text { Sulfate } \\
\left(\mathrm{SO}_{4}\right)\end{array}$} & \multirow{2}{*}{$\begin{array}{l}\text { Chlo- } \\
\text { ride } \\
\text { (C1) }\end{array}$} & \multirow{2}{*}{$\begin{array}{l}\text { Fluo } \\
\text { ride } \\
\text { (F) }\end{array}$} & \multirow{2}{*}{$\begin{array}{l}\mathrm{Ni}- \\
\text { trate } \\
\left(\mathrm{NO}_{3}\right)\end{array}$} & \multirow{2}{*}{$\begin{array}{l}\text { Bo- } \\
\text { ron } \\
\text { (B) }\end{array}$} & \multirow{2}{*}{$\begin{array}{c}\text { Dis- } \\
\text { solved } \\
\text { solids } \\
\text { (residue } \\
\text { on } \\
\text { evapo- } \\
\text { ration } \\
\text { at } \\
180^{\circ} \mathrm{C} \text { ) }\end{array}$} & \multicolumn{2}{|c|}{$\begin{array}{l}\text { Hardness } \\
\text { as } \\
\mathrm{CaCO}_{3}\end{array}$} & \multirow{2}{*}{\begin{tabular}{|} 
Per- \\
cent \\
sodi- \\
um
\end{tabular}} & \multirow{2}{*}{$\begin{array}{l}\text { Specific } \\
\text { con- } \\
\text { duct- } \\
\text { ance } \\
\text { (micro- } \\
\text { mhos } \\
\text { at } \\
\left.25^{\circ} \mathrm{C}\right)\end{array}$} & \multirow{2}{*}{$\mathrm{pH}$} & \multirow{2}{*}{$\begin{array}{c}\text { Source } \\
\text { of } \\
\text { data }\end{array}$} \\
\hline & & & & & & & & $\begin{array}{c}\text { Cal- } \\
\text { cium } \\
\text { mag- } \\
\text { nesium }\end{array}$ & $\begin{array}{l}\text { Non- } \\
\text { car- } \\
\text { bon- } \\
\text { ate }\end{array}$ & & & & \\
\hline 403 & 0 & 537 & 166 & & 2.0 . & & 1,143 & 878 & 548 & 21 & & & a \\
\hline 549 & 0 & 669 & 42 & & 3.8 & & 1,524 & 551 & 101 & 54 & & & a \\
\hline 378 & 0 & 374 & 472 & & 10 & & 1,678 & 88 & 0 & 93. & ........ & & a \\
\hline 200 & 0 & 1,790 & 30 & & 4 & & 2,660 & 1,444 & 1,280 & 29 & & & c \\
\hline 410 & 0 & 453 & 72 & & 33 & & 1,207 & 825 & 489 & 14 & & & a \\
\hline 535 & & 519 & 22 & 0 & 0 & & 1,246 & 543 & 104 & 46 & & & b \\
\hline 410 & 0 & 517 & 62 & & 40 & & 1,310 & 978 & 642 & 3 & & & $a$ \\
\hline 517 & ....... & 853 & 67 & 1.2 & 1.6 & & 1,840 & 167 & 0 & 89 & & & $b$ \\
\hline 515 & $\ldots \ldots$ & 820 & 72 & & 5.7 & & 1,688 & 764 & 342 & 44 & & & b \\
\hline 710 & $\ldots \ldots$ & 14 & 495 & 1.3 & 5.3 & & 1,497 & 130 & 0 & 90 & & & b \\
\hline 537 & 0 & 1,236 & 27 & & 20 & & 2,405 & $1 ; 600$ & 1,160 & 11 & & & a \\
\hline 463 & ........ & 978 & 22 & & 6.7 & & 1,986 & 1,175 & 795 & 11 & & & $\mathrm{~b}$ \\
\hline 405 & , & 1,082 & 178 & 0 & 8.8 & & 2,304 & 1,505 & 1,173 & 12 & & & b \\
\hline 410 & & 697 & 188 & .4 & 4.4 & & 1,869 & 1,239 & 903 & 7 & & & $\mathrm{~b}$ \\
\hline 520 & 0 & 763 & 44 & & 2.9 & & 1,650 & 614 & 188 & 52 & & & $\mathrm{a}$ \\
\hline 647 & 0 & 479 & 126 & & 1.6 & & 1,487 & 233 & 0 & 80 & & & $\mathrm{a}$ \\
\hline 300 & ....... & 1,058 & 328 & 1.4 & 6.7 & & 2,365 & 537 & 291 & 71 & & & $\vec{b}$ \\
\hline 327 & ........ & 1,116 & 354 & 4.0 & 4.0 & & 2,572 & 192 & 0 & 90 & & & $b$ \\
\hline 392 & $\cdots \ldots \ldots$ & 435 & 96 & 0 & 1.3 & & 1,169 & 686 & 365 & 25 & •.......... & $\ldots$ & $b$ \\
\hline 426 & ....... & 621 & 28 & .2 & 1.0 & & 1.323 & 409 & 60 & 61 & & & b \\
\hline 274 & $\ldots .$. & 848 & 265 & .6 & .35 & & 2,037 & 580 & 355 & 62 & & & b \\
\hline 314 & ...... & 914 & 275 & 3.6 & 7.1 & & 2,117 & 390 & 133 & 76 & & & $\mathrm{~b}$ \\
\hline 327 & ....... & 959 & 282 & 1.3 & .18 & & 2,178 & 371 & 103 & 79 & & & b \\
\hline 351 & $\ldots \ldots$ & 832 & 228 & 1.4 & .44 & $\ldots$ & 1,938 & 421. & 133 & 73 & •.......... & & $b$ \\
\hline 190 & 0 & 510 & 178 & & 80 & & 1,297 & 869 & 713 & 15 & & & a \\
\hline 571 & ...... & 482 & 152 & 0 & 3.1 & & 1,580 & 478 & 10 & 61 & ...... & & $\mathrm{b}$ \\
\hline 283 & ......... & 672 & 22 & .2 & 1.3 & & 1,268 & 386 & 154 & 60 & & & $\mathrm{~b}$ \\
\hline 529 & $\ldots .$. & 618 & 22 & .2 & .5 & & 1,338 & 474 & 40 & 59 & & & $\mathrm{~b}$ \\
\hline 403 & 0 & 856 & 14 & & 2.5 & & 1,601 & 962 & 632 & 23 & .. & & a \\
\hline 393 & $\ldots . .$. & 1,056 & 73 & 0 & 3.5 & & 2,029 & 953 & 631 & 38 & & & $b$ \\
\hline 366 & ...... & 1,031 & 392 & 0 & 425 & $\cdots \cdots \cdots$ & 2,950 & 1,605 & 1,305 & 29 & _.......... & ..... & $b$ \\
\hline 0 & 0 & 775 & 705 & .1 & 0 & & & 475 & 475 & 74 & & & c \\
\hline 478 & 0 & 783 & 218 & 0 & 13 & & & 545 & 153 & 50 & & & c \\
\hline 321 & 0 & 458 & 121 & .1 & 17 & & & 677 & 414 & 15 & & & c \\
\hline 451 & 0 & 790 & 106 & 0 & 48 & & & 1,230 & 860 & 11 & & & c \\
\hline 342 & 65 & 507 & 118 & .6 & 7 & & & 88 & 0 & 90 & - e. & ...ene & c \\
\hline 399 & 0 & 3,790 & 206 & 0 & 52 & & & 3,580 & 3,253 & 22 & & & c \\
\hline 178 & 0 & 1,080 & 320 & .1 & 217 & ....... & & 1,150 & 1,004 & 27 & •........ & & c \\
\hline 218 & 0 & 884 & 240 & .1 & 0 & ...... & & 665 & 486 & 57 & .0.e...... & & C \\
\hline 309 & 36 & 48 & 488 & 0 & 0 & _..... & .............. & 130 & 0 & 88 & ¿........... & & c \\
\hline 164 & 0 & 2,080 & 50 & $0^{1}$ & 26 & & & 1,370 & 1,236 & 15 & & & c \\
\hline
\end{tabular}


Table 6.-Chemical analyses of saline ground water from

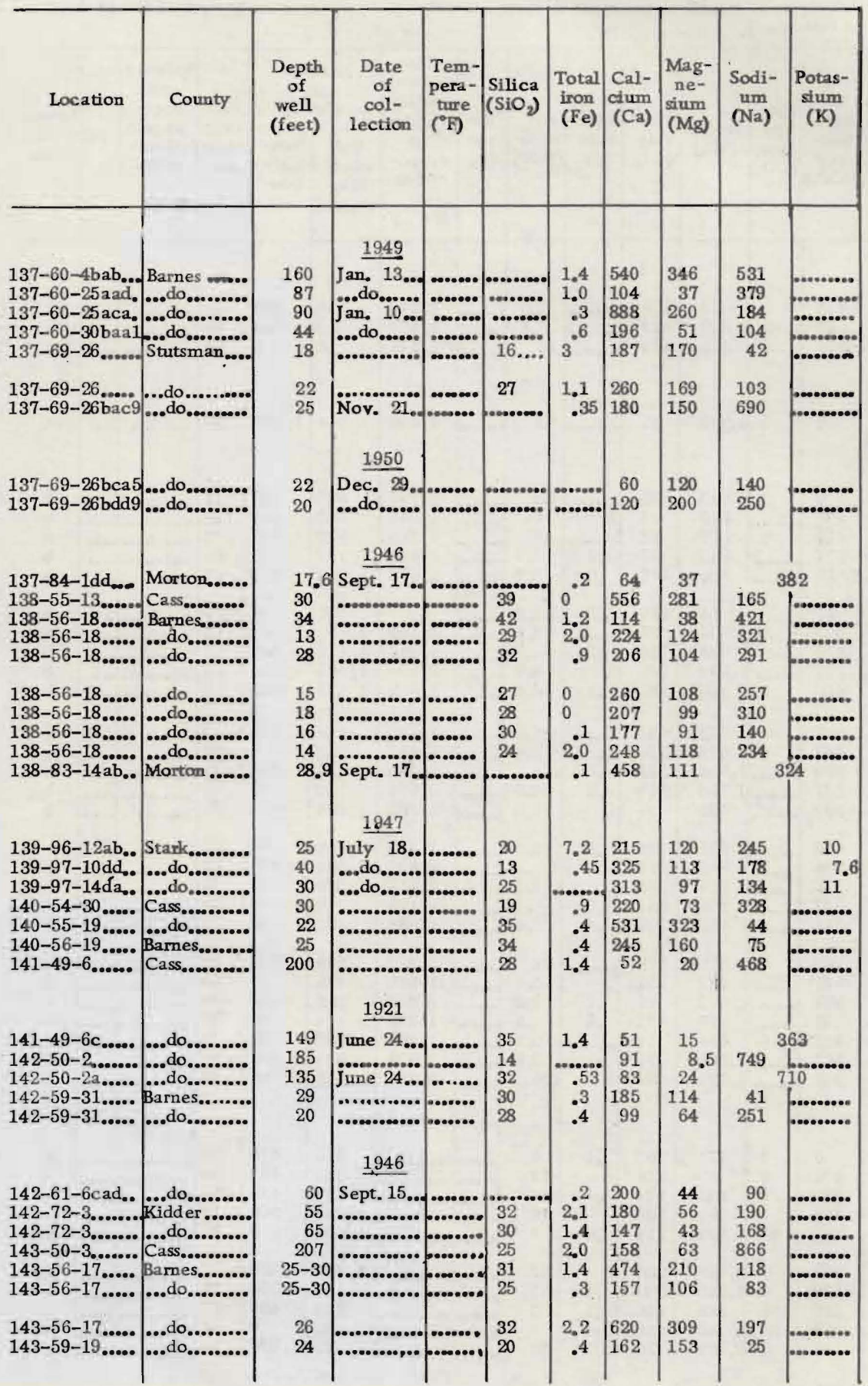


glacial drift and alluvium, North Dakota-Continued

\begin{tabular}{|c|c|c|c|c|c|c|c|c|c|c|c|c|c|}
\hline \multirow{2}{*}{$\begin{array}{c}\mathrm{Bi-} \\
\text { car- } \\
\text { bon- } \\
\text { ate } \\
\left(\mathrm{HCO}_{3}\right)\end{array}$} & \multirow{2}{*}{$\begin{array}{l}\text { Car- } \\
\text { bon- } \\
\text { ate } \\
\left(\mathrm{CO}_{3}\right)\end{array}$} & \multirow{2}{*}{$\begin{array}{l}\text { Sulfate } \\
\left(\mathrm{SO}_{4}\right)\end{array}$} & \multirow{2}{*}{$=\mid \begin{array}{l}\text { Chlo- } \\
\text { ride } \\
\text { (CI) }\end{array}$} & \multirow{2}{*}{$\begin{array}{l}\text { Fluo- } \\
\text { ride } \\
\text { (F) }\end{array}$} & \multirow{2}{*}{$\begin{array}{c}\mathrm{Ni}- \\
\text { trate } \\
\left(\mathrm{NO}_{3}\right)\end{array}$} & \multirow{2}{*}{$\begin{array}{l}\text { Bo- } \\
\text { ron } \\
\text { (B) }\end{array}$} & \multirow{2}{*}{$\begin{array}{c}\text { Dis- } \\
\text { solved } \\
\text { solids } \\
\text { (residue } \\
\text { on } \\
\text { evapo- } \\
\text { ration } \\
\text { at } \\
180^{\circ} \mathrm{C} \text { ) }\end{array}$} & \multicolumn{2}{|c|}{$\begin{array}{c}\text { Hardness } \\
\text { as } \\
\mathrm{CaCO}_{3}\end{array}$} & \multirow{2}{*}{$\begin{array}{l}\text { Per- } \\
\text { cent } \\
\text { sodi- } \\
\text { um }\end{array}$} & \multirow{2}{*}{$\begin{array}{c}\text { Specific } \\
\text { con- } \\
\text { duct- } \\
\text { ance } \\
\text { (micro- } \\
\text { mhos } \\
\text { at } \\
25^{\circ} \mathrm{C} \text { ) }\end{array}$} & \multirow{2}{*}{$\mathrm{pH}$} & \multirow{2}{*}{$\begin{array}{c}\text { Source } \\
\text { of } \\
\text { data }\end{array}$} \\
\hline & & & & & & & & $\begin{array}{c}\text { Cal- } \\
\text { cium, } \\
\text { mag- } \\
\text { nesium }\end{array}$ & $\begin{array}{l}\text { Non- } \\
\text { car- } \\
\text { bon- } \\
\text { ate }\end{array}$ & & & & \\
\hline 488 & 0 & 2,920 & 290 & 0 & 30 & & ............. & 2,780 & 2,380 & 29 & & & c \\
\hline 344 & 0 & 452 & 342 & 0 & 2 & & ............ & 410 & 128 & 67 & ............ & & c \\
\hline 494 & 0 & 748 & 1,780 & 0 & 4 & & . & ......... & $\ldots . .$. & 11 & ............ &... & c \\
\hline $\begin{array}{l}398 \\
710\end{array}$ & 0 & $\begin{array}{l}475 \\
411\end{array}$ & $\begin{array}{r}78 \\
78\end{array}$ & $\begin{array}{l}0 \\
.2\end{array}$ & $\begin{array}{r}0 \\
177\end{array}$ & & 1,511 & $\begin{array}{r}700 \\
1,171\end{array}$ & $\begin{array}{l}374 \\
589\end{array}$ & $\begin{array}{r}24 \\
7\end{array}$ & ….......... & $\ldots$ & $\begin{array}{l}\mathrm{c} \\
\mathrm{b}\end{array}$ \\
\hline 570 & & 631 & 242 & .2 & 133 & & 1,929 & 1,350 & 883 & 14 & & & b \\
\hline 570 & & 1,340 & 280 & & 390 & & -........... & 1,070 & 603 & 58 & ........ & $\cdot \cdots$ & c \\
\hline 340 & 17 & 590 & 47 & & 56 & & & 650 & 342 & 32 & & & c \\
\hline 270 & 24 & 1,110 & 210 & & 43 & & $\ldots \ldots$ & 1,140 & 878 & 33 & & & c \\
\hline 886 & 0 & 266 & 95 & 1.6 & .2 & & 1,400 & 312 & 0 & 73 & 2,060 & 7.6 & a \\
\hline 568 & & 2,146 & 174 & .2 & 6.6 & & 3,842 & 2,589 & 2,123 & 12 & & & $\mathrm{~b}$ \\
\hline $\begin{array}{l}587 \\
735\end{array}$ & & $\begin{array}{l}425 \\
580\end{array}$ & $\begin{array}{l}312 \\
411\end{array}$ & $0^{.2}$ & $\begin{array}{l}3.1 \\
6.2\end{array}$ & $\ldots$ & $\begin{array}{l}1,667 \\
2,148\end{array}$ & $\begin{array}{r}452 \\
1,093\end{array}$ & $\begin{array}{r}0 \\
490\end{array}$ & $\begin{array}{l}67 \\
40\end{array}$ & .............. & & $\begin{array}{l}b \\
b\end{array}$ \\
\hline 535 & & 660 & 321 & .2 & .8 & & 2,001 & 950 & 511 & 40 & 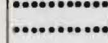 & . & $\mathrm{b}$ \\
\hline 381 & & 331 & 613 & 0 & 177 & & 2,024 & 1,105 & 793 & 34 & & & b \\
\hline 573 & & 667 & 304 & .2 & 5.3 & .... & $1: 988$ & 923 & 453 & 42 & & & b \\
\hline 495 & & 547 & 104 & 0 & 2.2 & & 1,456 & 818 & 412 & 27 & ............... & & b \\
\hline 600 & & 617 & 347 & .2 & .9 & ...... & 1,999 & 1,117 & 625 & 32 & ............. & & b \\
\hline 808 & 0 & 1,130 & 327 & .1 & 2.0 & ...... & 3,030 & 1,600 & 936 & 31 & 3,370 & 07.4 & a \\
\hline 505 & 0 & 1,100 & 10 & .5 & 1.5 & 0.26 & 2,160 & 1,030 & 616 & 34 & 2,340 & 07.3 & a \\
\hline 554 & 0 & 1,140 & 12 & .1 & 18 & & 2,300 & 1.280 & 826 & 23 & 2,320 & 07.1 & a \\
\hline 413 & 0 & 970 & 95 & .4 & 1.2 & ...... & 2,560 & 1,180 & 841 & 20 & 2,950 & 7.3 & a \\
\hline 451 & & 873 & 210 & .6 & 1.8 & $\ldots$ & 2,026 & 862 & 492 & 46 & .............. & .... & b \\
\hline 194 & ........ & 2,315 & 118 & .4 & 29 & ...... & 4,041 & 2,664 & 2,505 & 3 & ............. & & b \\
\hline 475 & $\ldots$ & 818 & 137 & 0 & 3.8 & $\ldots . .$. & 1,888 & 1,274 & 884 & 11 & ............. & & b \\
\hline 427 & $\ldots \ldots$ & 211 & 464. & .8 & 7.9 & -..... & 1,481 & 215 & 0 & 83 & $\ldots \ldots \ldots$ & ..... & b \\
\hline 390 & 0 & 193 & 328 & & 7.1 & & 1,194 & 189 & 0 & 81 & & & a \\
\hline 317 & & 435 & 830 & .8 & 6.4 & & 2,415 & 262 & 2 & 86 & $\ldots$ & & b \\
\hline 212 & 0 & 410 & 890 & & $15^{\circ}$ & $\ldots . .$. & 2,298 & 306 & 132 & 84 & ........ & & a \\
\hline 418 & & 375 & 202 & .2 & 2.7 & ...... & 1,238 & 931 & 588 & 9 & ............. &... & b \\
\hline 327 & & 377 & 284 & .2 & .4 & $\ldots . .$. & 1,358 & 516 & 248 & 52 & .............. & . .... & b \\
\hline 464 & & 418 & 17 & & & & & 680 & 300 & 22 & & & c \\
\hline 424 & & 629 & 67 & 0 & 1.8 & ...... & 1,438 & 686 & 338 & 38 & ............. & & b \\
\hline 512 & & 424 & 35 & 0 & 4.4 & $\ldots . .$. & 1,118 & 545 & 125 & 40 & ................. & & b \\
\hline 322 & & 897 & 946 & .8 & 11 & $\ldots$ & 3,196 & 660 & 396 & 74 & ............. & & b \\
\hline 351 & & 1,872 & 85 & .2 & 1.8 & ..... & 3,299 & 2,103 & 1,815 & 11 & ............. & $\ldots$ & b \\
\hline 381 & $\ldots \ldots$ & 613 & 39 & .2 & 4.4 & $\ldots$ & 1,324 & 825 & 513 & 18 & ........... & $\ldots$ & b \\
\hline 369 & & 2,621 & 156 & .2 & .4 & ...... & 4,701 & 2,825 & 2,522 & 13 & ............... & $\ldots$ & b \\
\hline 385 & & 588 & 73 & & 71 & & 1,469 & 1,034 & 718 & 5 & $\cdots \ldots \ldots$ & & b \\
\hline
\end{tabular}


Table 6. -Chemical analyses of saline ground water from

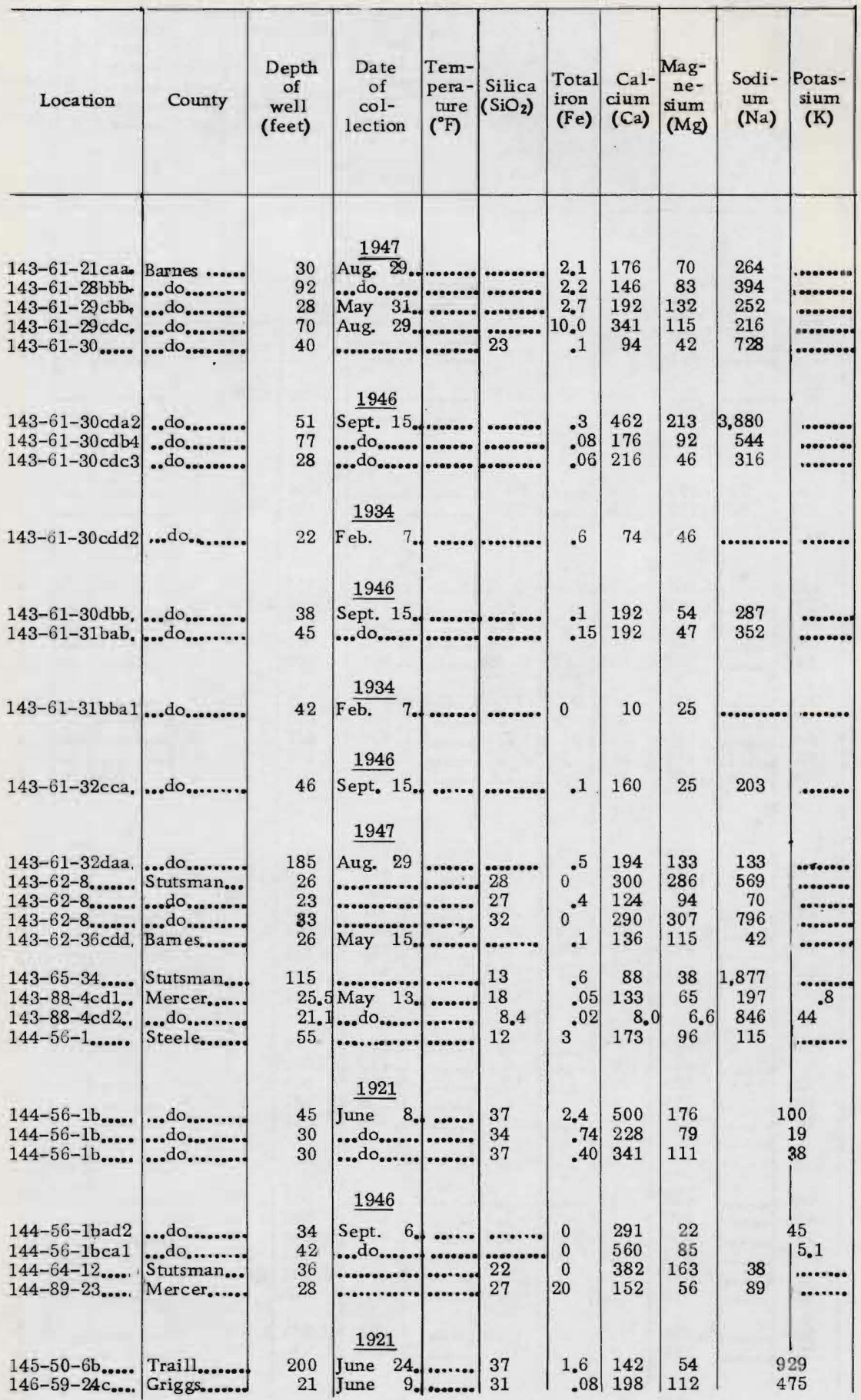


glacial drift and alluvium, North Dakota-Continued

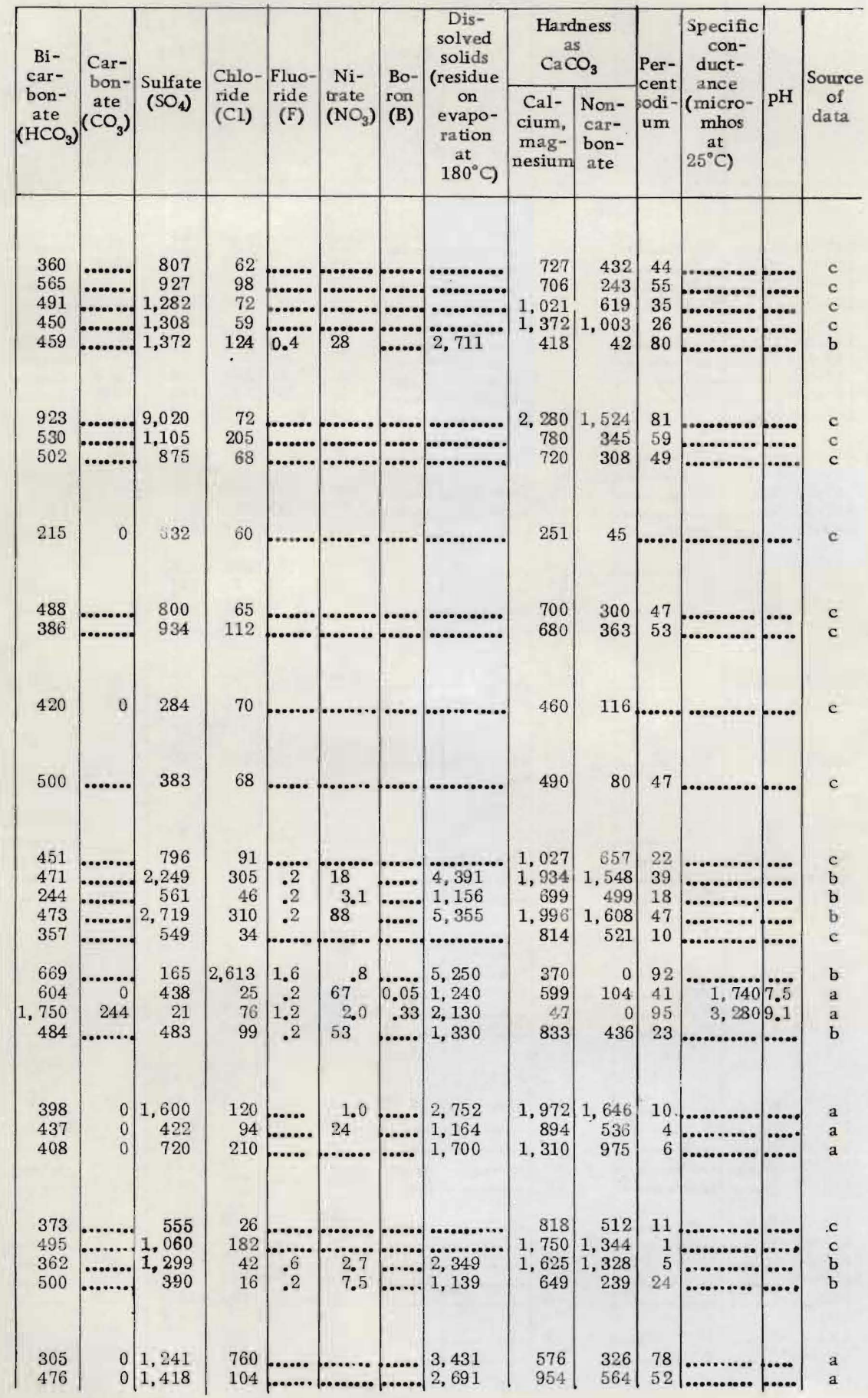

$4613970-58-4$ 
Table 6.-Chemical analyses of saline ground water from

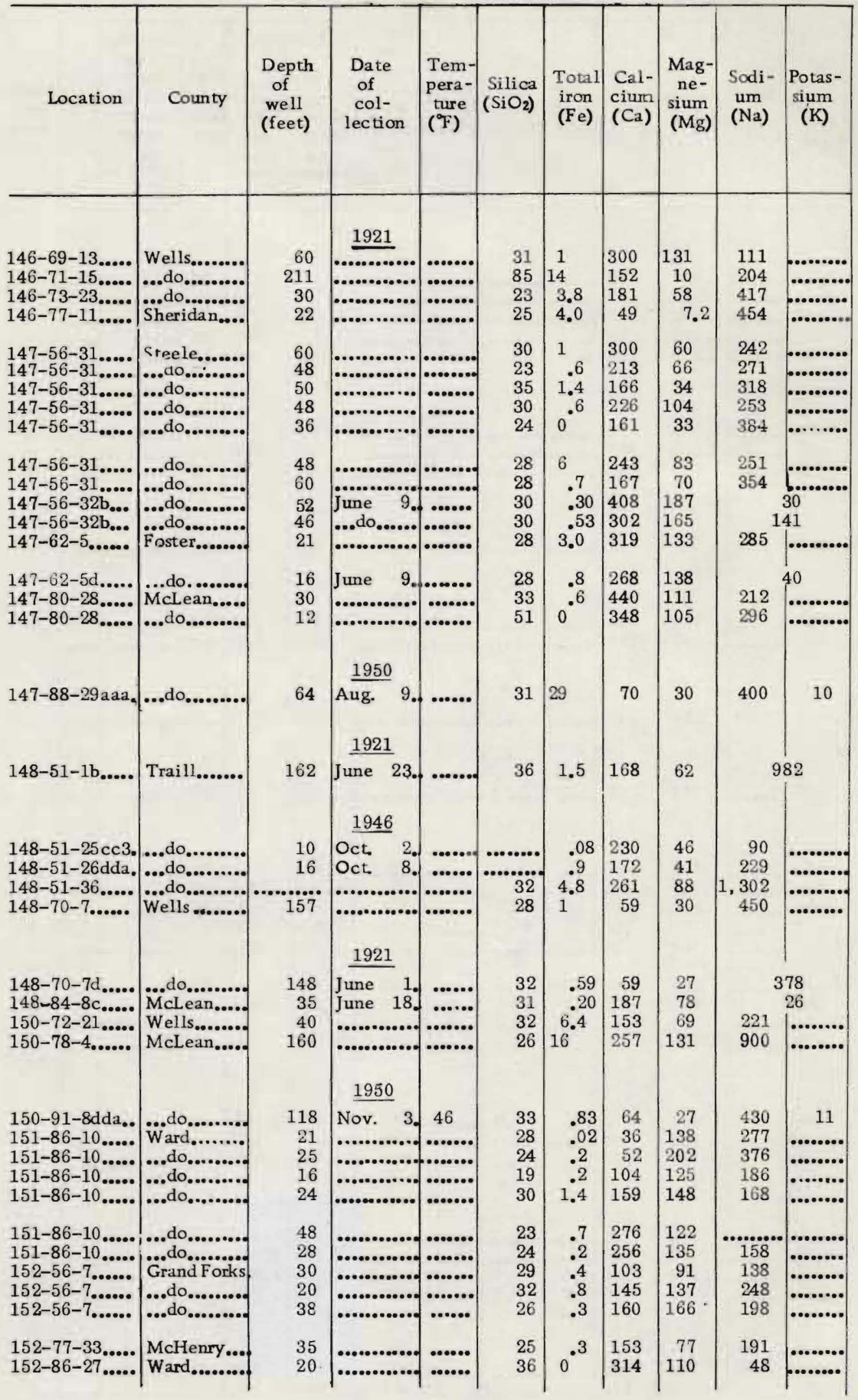


Blacial drift and alluvium, North Dakota-Continued

\begin{tabular}{|c|c|c|c|c|c|c|c|c|c|c|c|c|c|}
\hline \multirow{2}{*}{$\begin{array}{c}\text { Bi- } \\
\text { car- } \\
\text { bon- } \\
\text { ate } \\
\left(\mathrm{HCO}^{-}\right)\end{array}$} & \multirow{2}{*}{$\begin{array}{c}\text { Car- } \\
\text { bon- } \\
\text { ate } \\
\left(\mathrm{CO}_{3}\right)\end{array}$} & \multirow{2}{*}{$\begin{array}{l}\text { Sulfate } \\
\left(\mathrm{SO}_{4}\right)\end{array}$} & \multirow{2}{*}{$\begin{array}{l}\text { Chlo- } \\
\text { ride } \\
\text { (C) }\end{array}$} & \multirow{2}{*}{$\begin{array}{c}\text { Fluo- } \\
\text { ride } \\
\text { (F) }\end{array}$} & \multirow{2}{*}{$\begin{array}{c}\mathrm{Ni}- \\
\text { trate } \\
\left(\mathrm{NO}_{3}\right)\end{array}$} & \multirow{2}{*}{$\begin{array}{l}\text { Bo- } \\
\text { ron } \\
\text { (B) }\end{array}$} & \multirow{2}{*}{$\begin{array}{c}\text { Dis- } \\
\text { solved } \\
\text { solids } \\
\text { (residue } \\
\text { on } \\
\text { evapo- } \\
\text { ration } \\
\text { at } \\
180^{\circ} \mathrm{C} \text { ) }\end{array}$} & \multicolumn{2}{|c|}{$\begin{array}{c}\text { Hardness } \\
\text { as } \\
\mathrm{CaCO}_{3}\end{array}$} & \multirow{2}{*}{$\begin{array}{c}\text { Per- } \\
\text { cent } \\
\text { sodi- } \\
\text { um }\end{array}$} & \multirow{2}{*}{\begin{tabular}{|} 
Specific \\
con- \\
duct- \\
ance \\
(micro- \\
mhos \\
at \\
$25^{\circ} \mathrm{C}$ )
\end{tabular}} & \multirow[b]{2}{*}{$\mathrm{pH}$} & \multirow{2}{*}{$\begin{array}{c}\text { Source } \\
\text { of } \\
\text { data }\end{array}$} \\
\hline & & & & & & & & $\begin{array}{r}\text { Cal- } \\
\text { clum, } \\
\text { ma g- } \\
\text { nesipma }\end{array}$ & $\begin{array}{l}\text { Non- } \\
\text { car- } \\
\text { bon- } \\
\text { ate }\end{array}$ & & & & \\
\hline $\begin{array}{l}532 \\
688 \\
658 \\
842\end{array}$ & 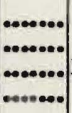 & $\begin{array}{r}617 \\
294 \\
1,002 \\
117\end{array}$ & $\begin{array}{r}321 \\
34 \\
24 \\
230\end{array}$ & $\begin{array}{l}0.6 \\
.8 \\
0 \\
3\end{array}$ & $\begin{array}{l}6.2 \\
2.6 \\
1 \\
3.5\end{array}$ & \begin{tabular}{c|}
$\ldots \ldots$ \\
$\ldots \ldots$ \\
$\ldots \ldots$ \\
$\ldots \ldots$
\end{tabular} & $\begin{array}{l}1,974 \\
1,185 \\
2,131 \\
1,271\end{array}$ & $\begin{array}{r}1,296 \\
479 \\
710 \\
160\end{array}$ & $\begin{array}{r}860 \\
0 \\
170 \\
0\end{array}$ & $\begin{array}{l}16 \\
51 \\
57 \\
87\end{array}$ & 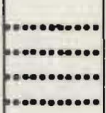 & $\mid$\begin{tabular}{c|}
$\cdots \cdots \bullet \bullet$ \\
$\cdots \cdots$ \\
$\cdots \cdots$ \\
$\cdots \cdots \bullet$
\end{tabular} & $\begin{array}{l}\mathbf{b} \\
\mathbf{b} \\
\mathbf{b} \\
\mathbf{b}\end{array}$ \\
\hline $\begin{array}{l}574 \\
571 \\
569 \\
549 \\
500\end{array}$ & $\begin{array}{c}\cdots \cdots \cdots \bullet \bullet \\
\cdots \cdots \cdots \bullet \bullet \\
\cdots \cdots \cdots \bullet \bullet \\
\cdots \cdots \cdots\end{array}$ & $\begin{array}{l}968 \\
891 \\
710 \\
994 \\
857\end{array}$ & $\begin{array}{l}36 \\
35 \\
30 \\
40 \\
50\end{array}$ & $\begin{array}{l}0 \\
.6 \\
.8 \\
.4 \\
.8\end{array}$ & $\begin{array}{r}18 \\
2.7 \\
6.2 \\
6.2 \\
2.7\end{array}$ & 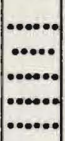 & $\begin{array}{l}2,038 \\
1,792 \\
1,596 \\
2,060 \\
1,801\end{array}$ & $\begin{array}{r}1,003 \\
815 \\
563 \\
992 \\
541\end{array}$ & $\begin{array}{r}532 \\
347 \\
96 \\
542 \\
131\end{array}$ & $\begin{array}{l}35 \\
42 \\
56 \\
36 \\
61\end{array}$ & 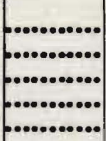 & $\mid$\begin{tabular}{c|}
$\ldots \ldots$ \\
$\cdots \ldots$ \\
$\cdots \cdots$ \\
$\cdots \cdots$ \\
$\cdots \cdots$
\end{tabular} & $\begin{array}{l}b \\
b \\
b \\
b \\
b\end{array}$ \\
\hline $\begin{array}{l}523 \\
484 \\
547 \\
464 \\
496\end{array}$ & {$\left[\begin{array}{r}\cdots \\
\cdots \\
\cdots\end{array}\right]$} & $\begin{array}{r}986 \\
950 \\
998 \\
1,232 \\
697\end{array}$ & $\begin{array}{r}36 \\
68 \\
213 \\
42 \\
593\end{array}$ & $\begin{array}{r}.8 \\
.2 \\
.6 \\
.6\end{array}$ & $\begin{array}{r}2.7 \\
8.7 \\
80 \\
10 \\
.9\end{array}$ & 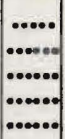 & $\begin{array}{l}1,964 \\
1,888 \\
2,522 \\
2,339 \\
2,411\end{array}$ & $\begin{array}{r}963 \\
710 \\
1,787 \\
1,431 \\
1,351\end{array}$ & $\begin{array}{r}534 \\
313 \\
1,338 \\
1,051 \\
944\end{array}$ & $\begin{array}{r}37 \\
52 \\
4 \\
18 \\
32\end{array}$ & 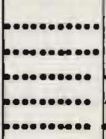 & 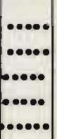 & $\begin{array}{l}b \\
b \\
a \\
a \\
b\end{array}$ \\
\hline $\begin{array}{l}400 \\
503 \\
718\end{array}$ & 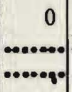 & $\begin{array}{r}430 \\
1,189 \\
526\end{array}$ & $\begin{array}{l}228 \\
144 \\
534\end{array}$ & $\begin{array}{c}\cdots \\
0 \\
0\end{array}$ & $\begin{array}{r}250 \\
212 \\
71\end{array}$ & & $\begin{array}{l}1,694 \\
2,807 \\
2,502\end{array}$ & $\begin{array}{l}1,240 \\
1,562 \\
1,360\end{array}$ & $\begin{array}{r}1,112 \\
1,150 \\
771\end{array}$ & $\begin{array}{r}7 \\
23 \\
33\end{array}$ & م.......... & & $\begin{array}{l}a \\
b \\
b\end{array}$ \\
\hline 856 & 0 & 430 & 20 & .4 & 3.8 & 0.20 & 1,500 & 298 & 0 & 74 & 2,030 & 7.4 & a \\
\hline 290 & 0 & 1.270 & 860 & & & $\ldots$ & 3,660 & 674 & 436 & 76 & "......ecese. & & a \\
\hline $\begin{array}{l}402 \\
856 \\
312 \\
749\end{array}$ & $\begin{array}{r}\cdots \\
0 \\
\cdots \cdots \cdots \\
\cdots \cdots \cdots\end{array}$ & $\begin{array}{r}108 \\
801 \\
1,340 \\
359\end{array}$ & $\begin{array}{r}375 \\
518 \\
1,551 \\
190\end{array}$ & 0 & $\begin{array}{c}\cdots \cdots \\
0.7 \\
9 .\end{array}$ & $\mid$\begin{tabular}{c|c}
$\cdots \ldots$ \\
$\ldots \ldots$ \\
$\ldots \ldots$ \\
$\ldots \ldots$
\end{tabular} & $\begin{array}{l}4,825 \\
1,494\end{array}$ & $\begin{array}{r}764 \\
598 \\
1,020 \\
285\end{array}$ & $\begin{array}{r}434 \\
0 \\
764 \\
0\end{array}$ & $\begin{array}{l}20 \\
45 \\
74 \\
78\end{array}$ & - & & $\begin{array}{l}c \\
c \\
b \\
b\end{array}$ \\
\hline $\begin{array}{l}651 \\
490 \\
651 \\
766\end{array}$ & $\begin{array}{r}0 \\
0 \\
0 \\
\cdots \cdots\end{array}$ & $\begin{array}{r}309 \\
422 \\
585 \\
2,285\end{array}$ & $\begin{array}{r}170 \\
10 \\
37 \\
111\end{array}$ & $0^{.4}$ & $\begin{array}{c}\cdots .6 \\
2.6 \\
2.7 \\
6.7\end{array}$ & & $\begin{array}{l}1,321 \\
1,052 \\
1,460 \\
4,271\end{array}$ & $\begin{array}{r}258 \\
788 \\
680 \\
1,212\end{array}$ & $\begin{array}{r}0 \\
386 \\
146 \\
584\end{array}$ & $\begin{array}{r}76 \\
7 \\
42 \\
62\end{array}$ & 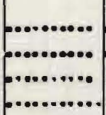 & & $\begin{array}{l}a \\
a \\
b \\
b\end{array}$ \\
\hline $\begin{array}{l}854 \\
444 \\
532 \\
462 \\
349\end{array}$ & 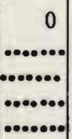 & $\begin{array}{l}500 \\
436 \\
849 \\
633 \\
386\end{array}$ & $\begin{array}{r}16 \\
285 \\
413 \\
42 \\
284\end{array}$ & $\begin{array}{l}0 \\
0 \\
.8 \\
.8\end{array}$ & $\begin{array}{c}3.1 \\
53 \\
1.8 \\
106 \\
354\end{array}$ & 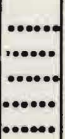 & $\begin{array}{l}1,520 \\
1,509 \\
2,385 \\
1,446 \\
1,931\end{array}$ & $\begin{array}{r}271 \\
661 \\
965 \\
780 \\
1,010\end{array}$ & $\begin{array}{r}0 \\
297 \\
529 \\
401 \\
724\end{array}$ & $\begin{array}{l}77 \\
48 \\
46 \\
34 \\
27\end{array}$ & 2,160 & 7.8 & $\begin{array}{l}a \\
b \\
b \\
b \\
b\end{array}$ \\
\hline $\begin{array}{l}344 \\
378 \\
556 \\
581 \\
624\end{array}$ & 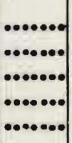 & $\begin{array}{l}569 \\
402 \\
293 \\
732 \\
668\end{array}$ & $\begin{array}{r}156 \\
173 \\
50 \\
104 \\
140\end{array}$ & $\begin{array}{l}.6 \\
0 \\
0 \\
0 \\
0\end{array}$ & $\begin{array}{c}44 \\
708 \\
2.7 \\
106 \\
.9\end{array}$ & 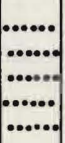 & $\begin{array}{l}1,461 \\
2,111 \\
1,128 \\
1,851 \\
1,969\end{array}$ & $\begin{array}{r}1,195 \\
1,200 \\
632 \\
930 \\
1,083\end{array}$ & $\begin{array}{l}913 \\
890 \\
176 \\
454 \\
571\end{array}$ & $\begin{array}{l}22 \\
32 \\
37 \\
28\end{array}$ & 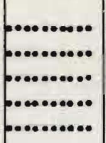 & 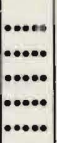 & $\begin{array}{l}b \\
b \\
b \\
b \\
b\end{array}$ \\
\hline $\begin{array}{l}390 \\
495\end{array}$ & 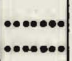 & $\begin{array}{l}596 \\
798\end{array}$ & $\begin{array}{l}77 \\
67\end{array}$ & .2 & $\begin{array}{l}80 \\
18\end{array}$ & ....... & $\begin{array}{l}1,342 \\
1,810\end{array}$ & $\begin{array}{r}699 \\
1,243\end{array}$ & $\begin{array}{l}379 \\
837\end{array}$ & $\begin{array}{r}37 \\
8\end{array}$ & & ..... & $\begin{array}{l}b \\
b\end{array}$ \\
\hline
\end{tabular}


Table 6.-Chemical analyses of saline ground water from

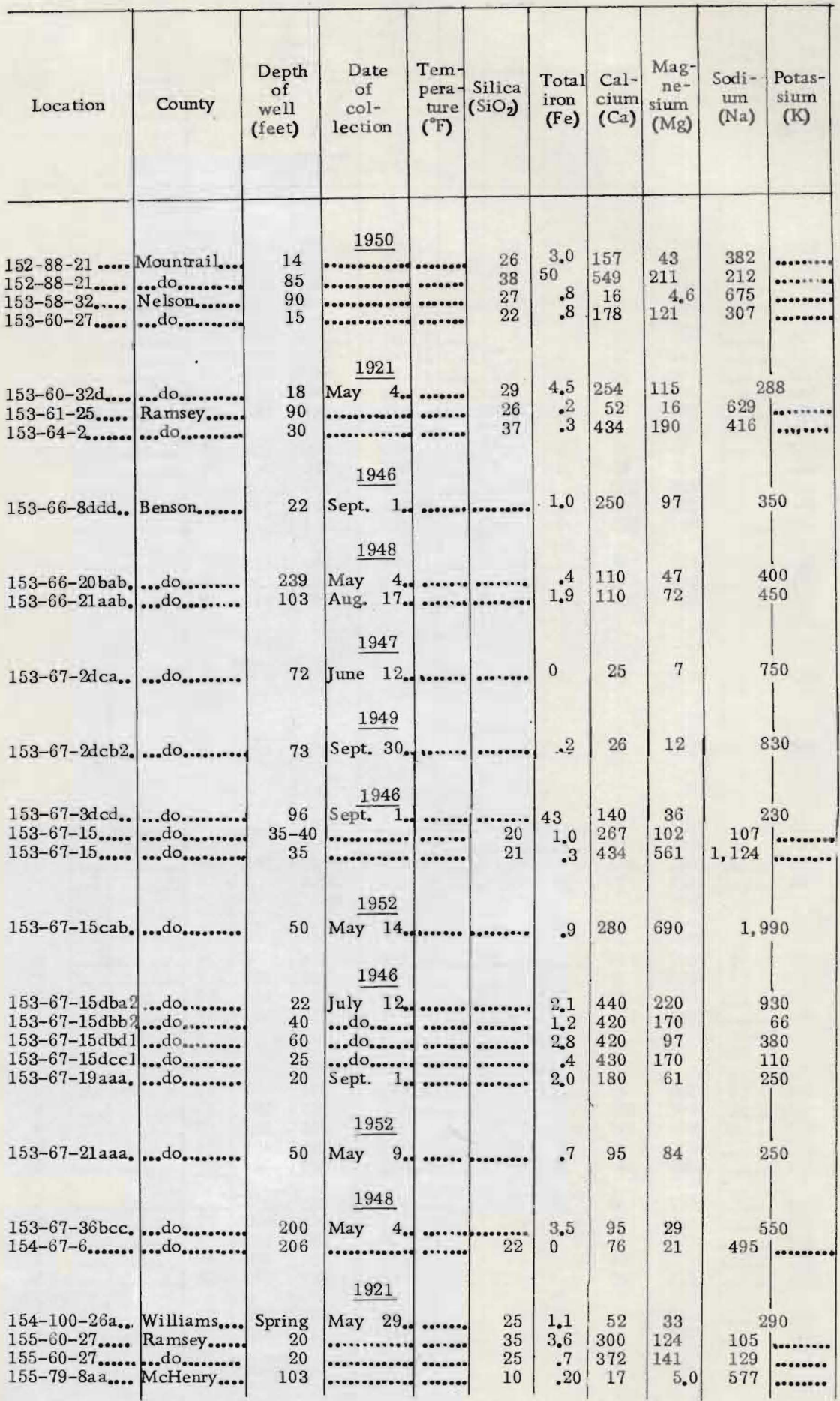


glacial drift and alluvium, North Dakota-Continued

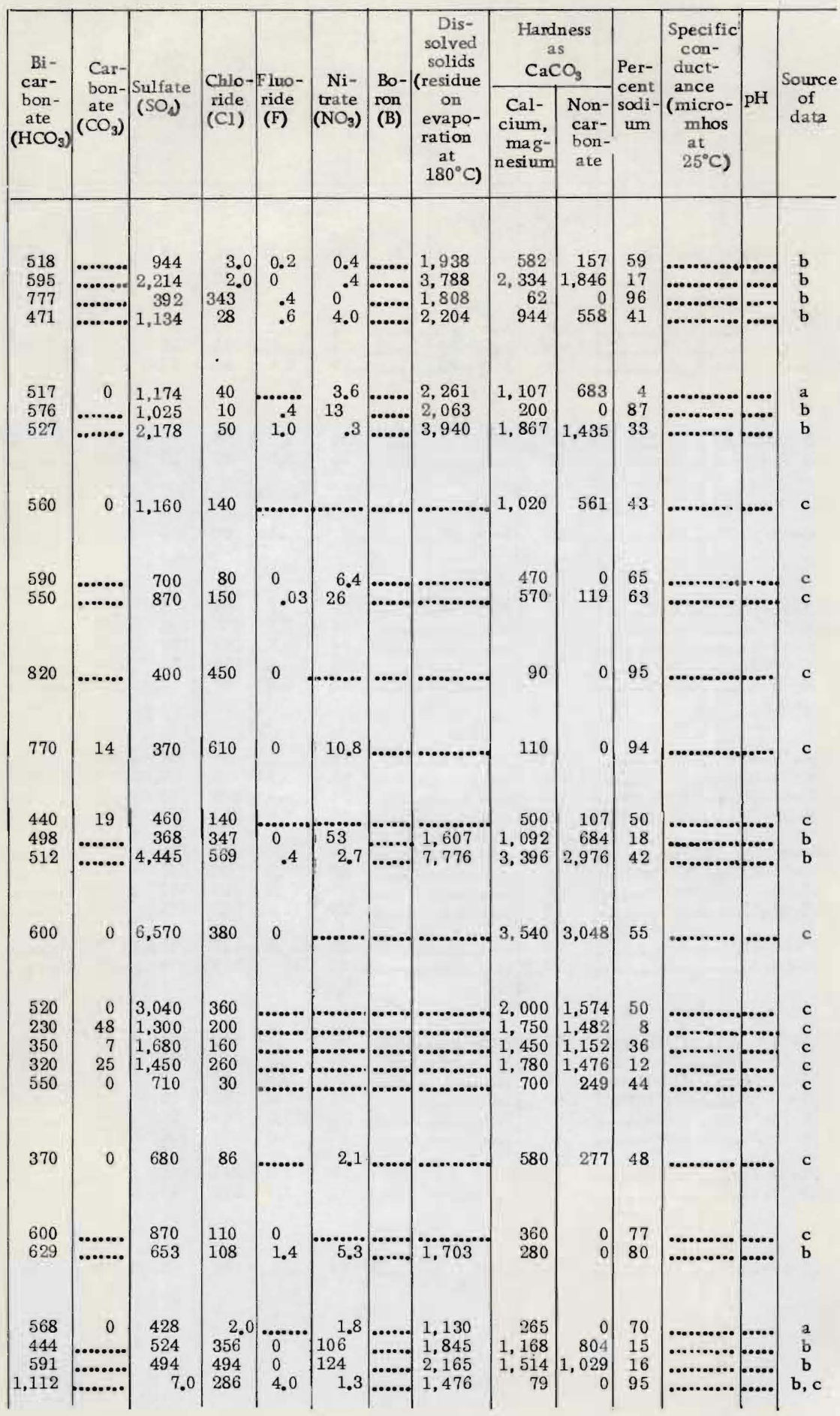


Table 6.-Chemical analyses of saline ground water from

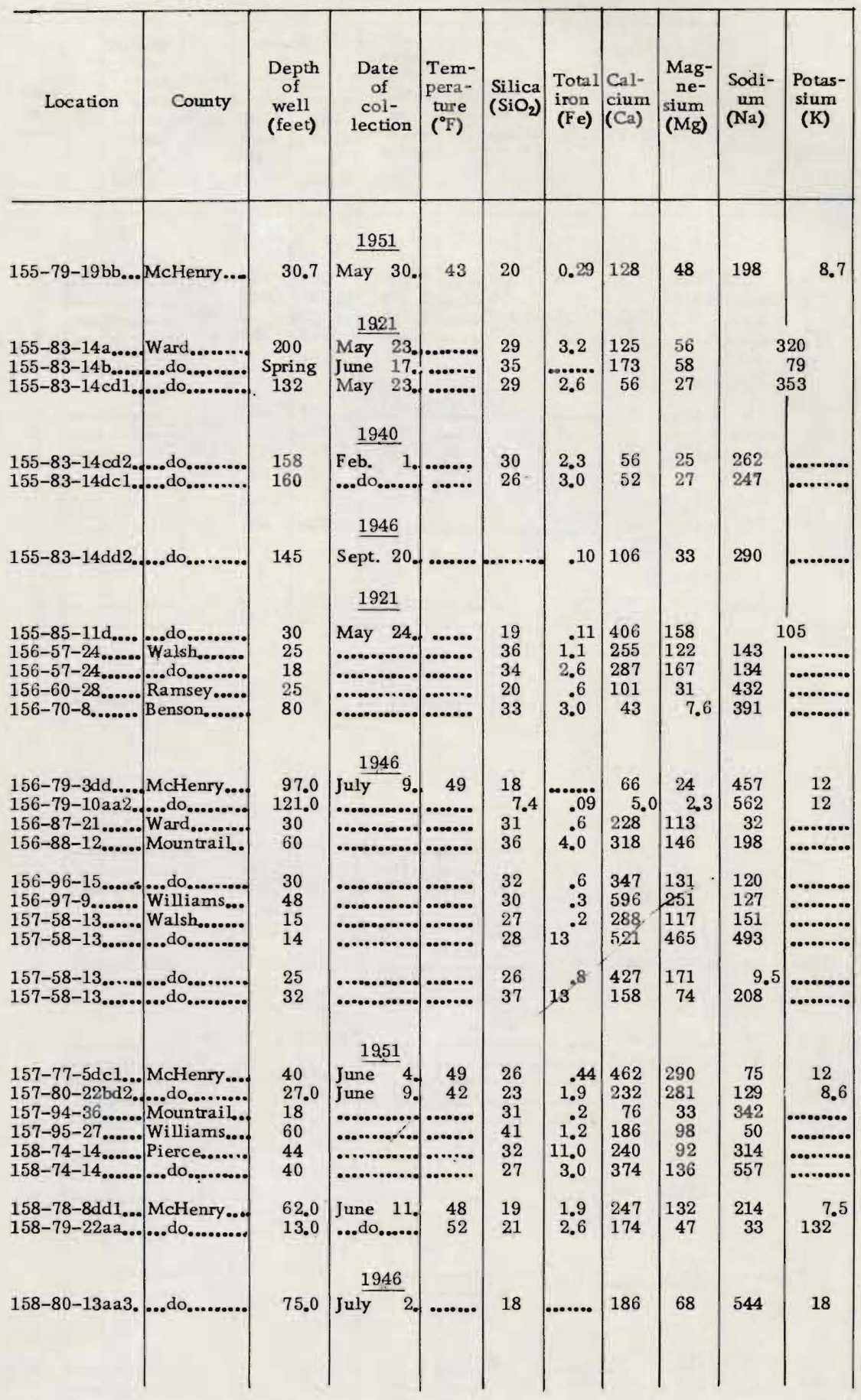


glacial drift and alluvium, North Dakota-Continued

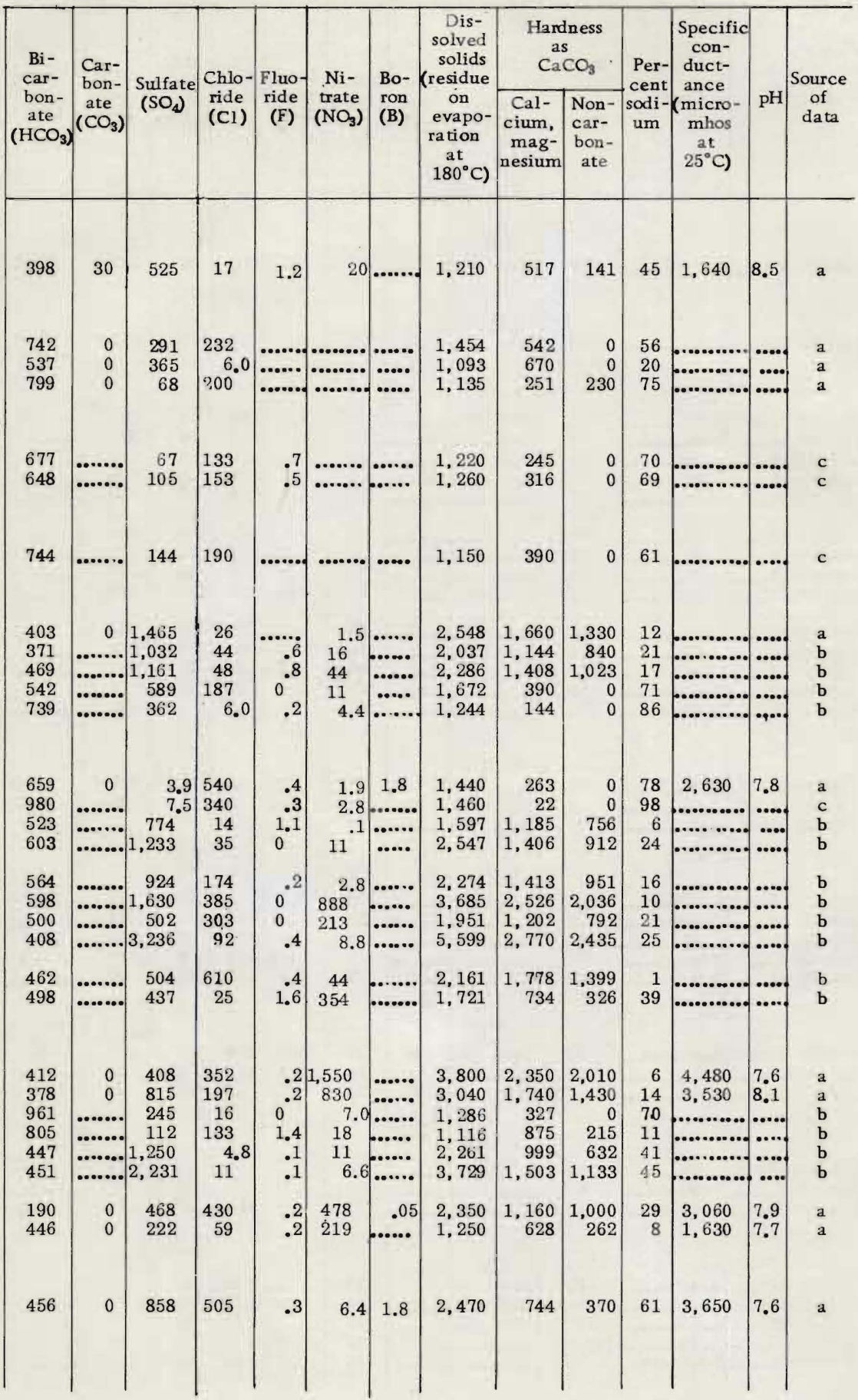


Table 6. - Chemical analyses of saline ground water from

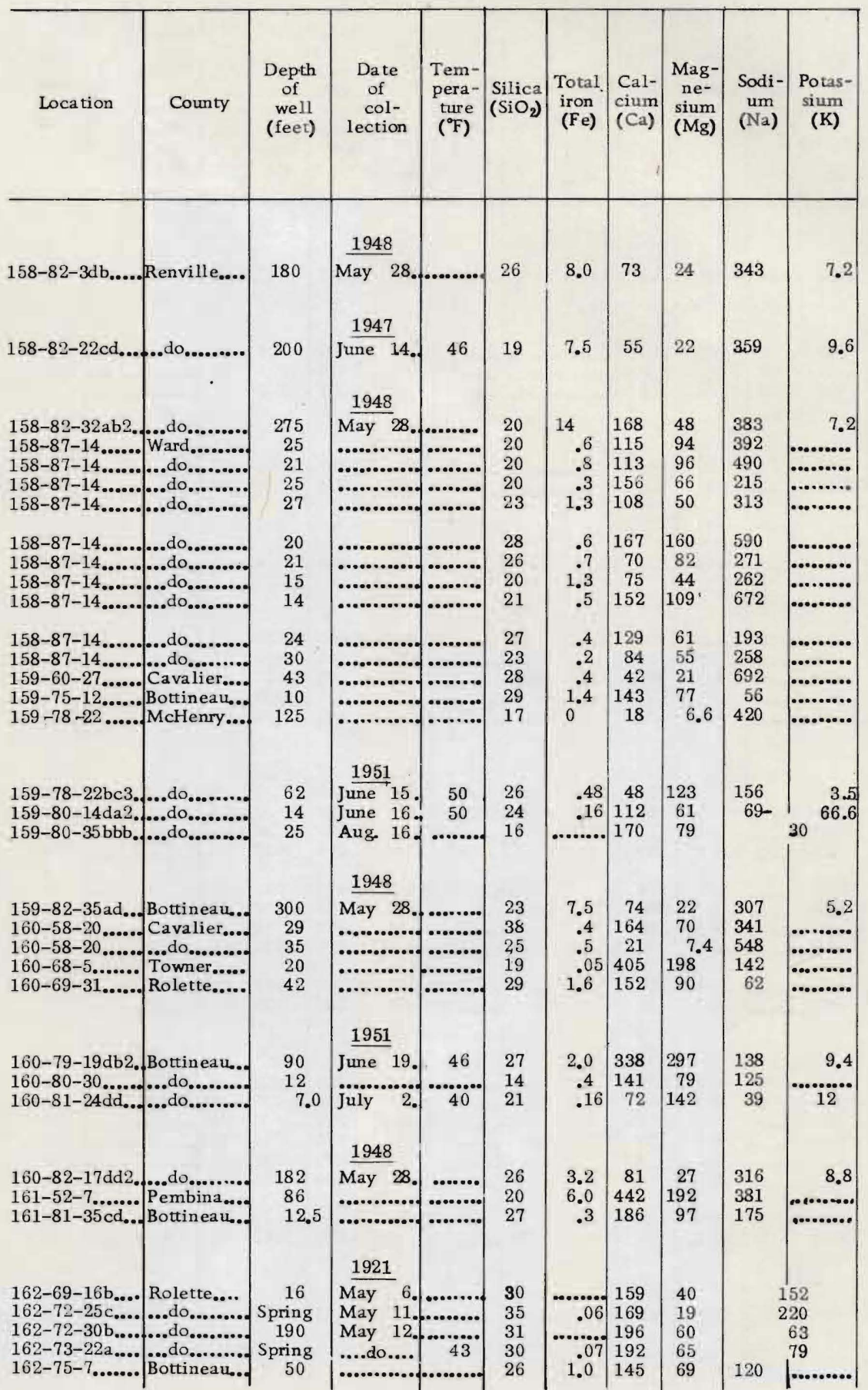


glacial drift and alluvium, North Dakota-Continued

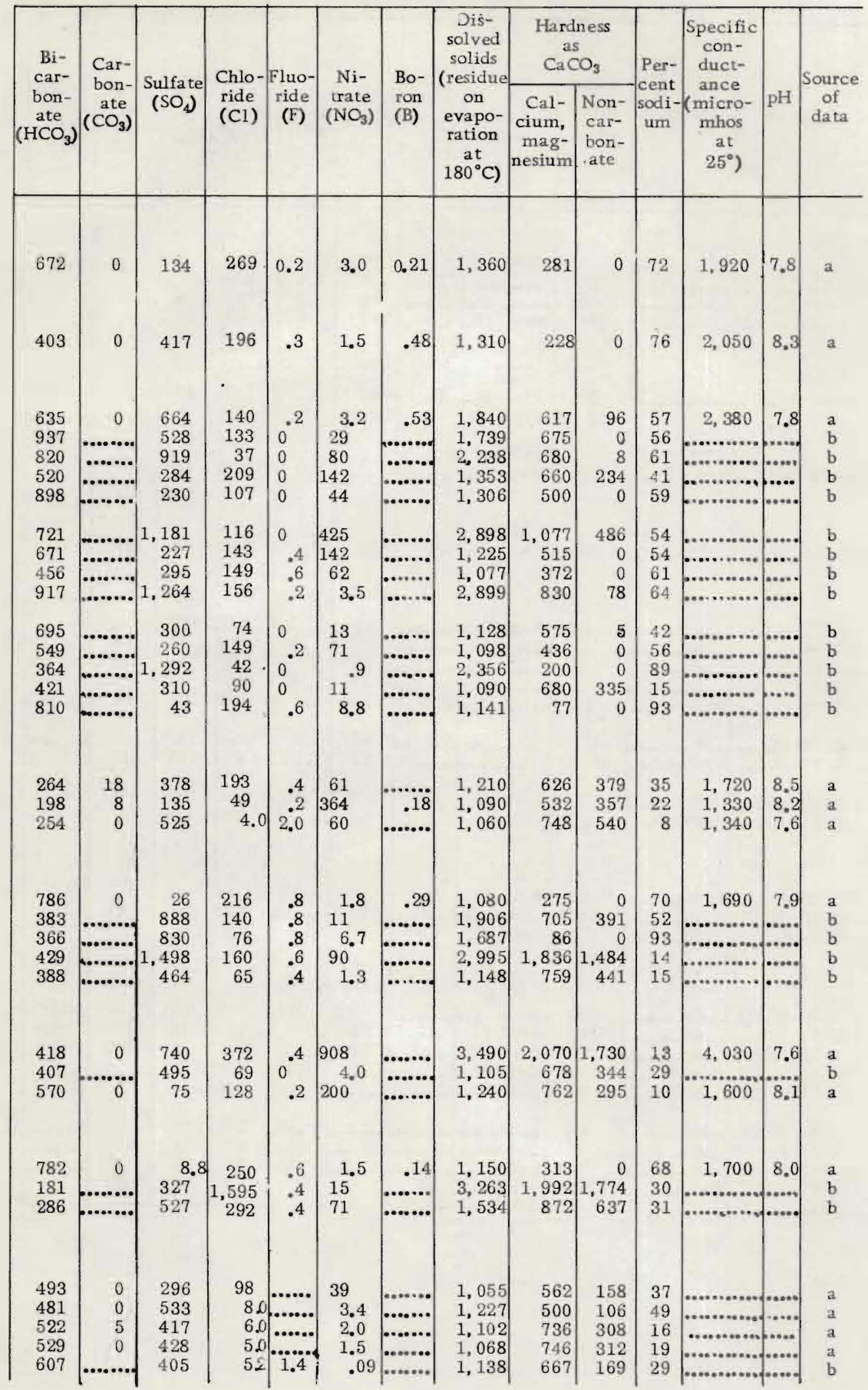


Table 6. - Chemical analyses of saline ground water from

\begin{tabular}{|c|c|c|c|c|c|c|c|c|c|c|}
\hline Location & County & $\begin{array}{c}\text { Depth } \\
\text { of } \\
\text { well } \\
\text { (feet) }\end{array}$ & $\begin{array}{l}\text { Date } \\
\text { of } \\
\text { col- } \\
\text { lection }\end{array}$ & $\begin{array}{l}\text { Tem- } \\
\text { pera- } \\
\text { ture } \\
\left({ }^{\circ} \mathrm{F}\right)\end{array}$ & $\begin{array}{l}\text { Silica } \\
\left(\mathrm{SiO}_{2}\right)\end{array}$ & $\begin{array}{c}\text { Total } \\
\text { iron } \\
(\mathrm{Fe})\end{array}$ & $\begin{array}{l}\text { Cal- } \\
\text { cium } \\
(\mathrm{Ca})\end{array}$ & $\begin{array}{c}\text { Mag- } \\
\text { ne- } \\
\text { sium } \\
\text { (Mg) }\end{array}$ & $\begin{array}{c}\text { Sodi- } \\
\text { um } \\
(\mathrm{Na})\end{array}$ & $\begin{array}{l}\text { Potas- } \\
\text { sium } \\
(\mathrm{K})\end{array}$ \\
\hline & Bottineau & 50 & $\underline{1921}$ & ......... & 30 & 1.4 & 229 & 87 & 0 & \\
\hline $\begin{array}{l}162-75-7 \ldots \ldots . . . \\
162-75-15 c_{.} . . .\end{array}$ & ...do.......... & 50 & .............. & ......... & 50 & .2 & 223 & 85 & & ...... \\
\hline $162-75-15 c_{. . . .}$ & ...do............... & 14 & May 16. & ......... & 14 & .67 & 49 & 19 & & 92 \\
\hline $162-81-11 \mathrm{bcl}$. & do........... & 30 & $\frac{1951}{\text { July }} 3 .$. & 43 & 20 & .10 & 139 & 79 & 31 & 8.9 \\
\hline $162-88-34$ dc... & Burke & 320 & May $29 .$. & ......... & 2.9 & 3.0 & 271 & 80 & 440 & 7.2 \\
\hline $162-89-3 \mathrm{ad} . .$. & do......... & 182 & June $5 .$. & 50 & 25 & 30 & 93 & 44 & 391 & 17 \\
\hline $162-90-10 \mathrm{cbc}$ & ...do & 22.5 & $\frac{1951}{\text { Aug. } 28 . .}$ & & 16 & & $407 k$ & 2,410 & 2,72 & \\
\hline $162-94$ & & $26^{\circ}$ & ................... & ......... & 30 & .36 & 228 & 67 & 82 & ..... \\
\hline $162-94$ & $\ldots$ do & 30 & & …....... & 27 & 0 & 63 & 64 & 309 & \\
\hline $162-94-3 a \mathrm{~d} 2$. & ....do. & 26 & 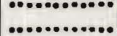 & (............. & 30 & .36 & 228 & 67 & & 32 \\
\hline $163-70-15 \ldots .$. & Rolette..... & 16 & .............. & ……... & 20 & 1.0 & 154 & 307 & 309 & ......... \\
\hline $163-70-15 . .$. & $\ldots$ do. & 155 & .... & .......... & 36 & 1.0 & 364 & 159 & 76 & .• \\
\hline $163-77-29$ & Bottin & $45-50$ & & ........ & 25 & 3.0 & 353 & 126 & 47 & ..... $>$ \\
\hline $\begin{array}{l}163-80-5 d c . . . \\
163-84-6 \text { ad.... }\end{array}$ & ....do.......... & $\begin{array}{l}20 \\
22\end{array}$ & June 25. & 44 & 22 & .63 & $\begin{array}{l}336 \\
337\end{array}$ & $\begin{array}{r}143 \\
1370\end{array}$ & $\begin{array}{ll}46 & \\
1,21 & \end{array}$ & 43 \\
\hline & & & 1947 & & & & & & & \\
\hline $163-88-4 \mathrm{bb} . \ldots$ & Burke....... & 120 & June 12. & 45 & 12 & .60 & 44 & 9.8 & 653 & 12 \\
\hline $\begin{array}{l}163-88-24 \mathrm{bbl} . \\
163-89-8 \mathrm{ad} . . . .\end{array}$ & ...dodo............ & $\begin{array}{l}440 \\
153\end{array}$ & June...... & $\begin{array}{l}50 \\
50\end{array}$ & $\begin{array}{l}23 \\
13\end{array}$ & & $\begin{array}{r}423 \\
45\end{array}$ & $\begin{array}{r}307 \\
21\end{array}$ & $\begin{array}{l}245 \\
729\end{array}$ & $\begin{array}{r}23 \\
3.2\end{array}$ \\
\hline & & & $\underline{1948}$ & & & & & & & \\
\hline $163-89-25 \mathrm{ad}$. & ...do... & 200 & June 2. & & 2.5 & .80 & 36 & 21 & 978 & 15 \\
\hline & ...do, & 140 & Sept, $11 .$. & 49 & 25 & & 195 & 45 & 207 & 2.8 \\
\hline & 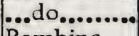 & 200 & June $2 .$. & & 12 & & 348 & 103 & 402 & 12 \\
\hline $\begin{array}{l}164-53-31 \mathrm{cab} \\
164-53-31 \mathrm{cbc} 2\end{array}$ & Pembina.... & $\begin{array}{l}23 \\
20\end{array}$ & $\begin{array}{l}\text { Oct. } 23 . . \\
\ldots \text {.......... }\end{array}$ & & ........ & $\begin{array}{l}1.5 \\
.15\end{array}$ & $\begin{array}{l}400 \\
400\end{array}$ & $\begin{array}{l}320 \\
150\end{array}$ & & $0^{\cdots \cdots \cdots}$ \\
\hline $164-53-31 \mathrm{ccb}$ & ...do... & 13 & & & & .45 & 560 & 230 & 35 & \\
\hline & ....do.. & 20 & & 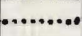 & $\ldots . . . .$. & .53 & 420 & 73 & 170 & .............. \\
\hline $\begin{array}{l}164-53-31 \mathrm{dcc} \\
164-92-34 \mathrm{dc} 2\end{array}$ & 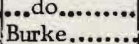 & $\begin{array}{l}20 \pm \\
345\end{array}$ & ...do...... & $\dddot{44}$ & 8.0 & $41^{.88}$ & $\begin{array}{r}180 \\
33\end{array}$ & $\begin{array}{l}63 \\
58\end{array}$ & $\begin{array}{r}45 \\
948\end{array}$ & ${ }_{12}$ \\
\hline
\end{tabular}


glacial drift and alluvium, North Dakota-Continued

\begin{tabular}{|c|c|c|c|c|c|c|c|c|c|c|c|c|c|}
\hline \multirow{2}{*}{$\begin{array}{c}\mathrm{Bi}- \\
\mathrm{car}- \\
\text { bon- } \\
\text { ate } \\
\left(\mathrm{HCO}_{3}\right)\end{array}$} & \multirow{2}{*}{$\begin{array}{l}\text { Car- } \\
\text { bon- } \\
\text { ate } \\
\left(\mathrm{CO}_{3}\right)\end{array}$} & \multirow{2}{*}{$\begin{array}{l}\text { Sulfate } \\
\left(\mathrm{SO}_{4}\right)\end{array}$} & \multirow{2}{*}{$\begin{array}{l}\text { Chlo- } \\
\text { ride } \\
\text { (Cl) }\end{array}$} & \multirow{2}{*}{$\begin{array}{l}\text { luo- } \\
\text { ride } \\
\text { (F) }\end{array}$} & \multirow{2}{*}{$\begin{array}{c}\mathrm{Ni}- \\
\text { trate } \\
\left(\mathrm{NO}_{3}\right)\end{array}$} & \multirow{2}{*}{$\begin{array}{l}\text { Bo- } \\
\text { ron } \\
\text { (B) }\end{array}$} & \multirow{2}{*}{$\begin{array}{c}\text { Dis- } \\
\text { solved } \\
\text { solids } \\
\text { (residue } \\
\text { on } \\
\text { evapo- } \\
\text { ration } \\
\text { at } \\
180^{\circ} \mathrm{C}\end{array}$} & \multicolumn{2}{|c|}{$\begin{array}{c}\text { Hardness } \\
\text { as } \\
\mathrm{CaCO}_{3}\end{array}$} & \multirow{2}{*}{\begin{tabular}{|} 
Per- \\
cent \\
sodi- \\
um
\end{tabular}} & \multirow{2}{*}{$\begin{array}{c}\text { Specific } \\
\text { con- } \\
\text { duct- } \\
\text { ance } \\
\text { (micro- } \\
\text { mhos } \\
\text { at } \\
\left.25^{\circ} \mathrm{C}\right)\end{array}$} & \multirow[b]{2}{*}{$\mathrm{pH}$} & \multirow{2}{*}{$\begin{array}{c}\text { Source } \\
\text { of } \\
\text { data }\end{array}$} \\
\hline & & & & & & & & $\begin{array}{c}\text { Cal- } \\
\text { cium, } \\
\text { ma.g- } \\
\text { nesium }\end{array}$ & $\begin{array}{c}\text { Non- } \\
\text { car- } \\
\text { bon- } \\
\text { ate }\end{array}$ & & & & \\
\hline $\begin{array}{l}376 \\
583 \\
688\end{array}$ & $\cdots$ & $\begin{array}{l}405 \\
444 \\
656\end{array}$ & $\begin{array}{r}8.0 \\
8.5 \\
12\end{array}$ & $\begin{array}{c}0 \\
0 \\
\ldots . .\end{array}$ & $\begin{array}{r}1.8 \\
1.8 \\
\ldots . . .\end{array}$ & & $\begin{array}{l}1,112 \\
1,141 \\
1,648\end{array}$ & $\begin{array}{l}930 \\
905 \\
200\end{array}$ & $\begin{array}{r}622 \\
427 \\
0\end{array}$ & $\begin{array}{r}0 \\
84\end{array}$ & $\mid \begin{array}{c}\ldots \ldots \ldots \ldots \ldots \\
\ldots \ldots \ldots \ldots \ldots\end{array}$ & & $\begin{array}{l}b \\
b \\
a\end{array}$ \\
\hline 276 & 14 & 180 & 44 & .2 & 300 & & 1,000 & 672 & 423 & 9 & 1,330 & 8.3 & a \\
\hline 478 & 0 & 1,430 & 42 & .4 & 4.4 & 0.21 & 2,680 & 1,010 & 618 & 48 & 3,010 & 7.2 & a \\
\hline 576 & 33 & 593 & 72 & .6 & 3.0 & .55 & 1,550 & 413 & 0 & 66 & 2,090 & 8.1 & a \\
\hline $\begin{array}{l}805 \\
487 \\
330 \\
487 \\
944\end{array}$ & 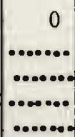 & $\begin{array}{r}15,400 \\
144 \\
659 \\
144 \\
532\end{array}$ & $\begin{array}{r}107 \\
320 \\
93 \\
320 \\
646\end{array}$ & $\begin{array}{l}2.0 \\
1.6 \\
2.2 \\
1.6 \\
1.0\end{array}$ & $\begin{array}{c}1.1 \\
22 \\
3.5 \\
22 \\
106\end{array}$ & $\begin{array}{c}\ldots . . . \\
\ldots \ldots . . \\
\ldots \ldots . . . \\
\ldots . . .\end{array}$ & $\begin{array}{r}24,100 \\
1,109 \\
1,447 \\
1,109 \\
2,683\end{array}$ & $\begin{array}{r}10,900 \\
844 \\
422 \\
844 \\
1,654\end{array}$ & $\begin{array}{r}10,200 \\
445 \\
151 \\
445 \\
880\end{array}$ & $\begin{array}{l}35 \\
17 \\
62 \\
17 \\
29\end{array}$ & 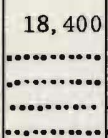 & $\begin{array}{c}7.8 \\
\cdots \ldots . \\
\cdots \cdots \\
\ldots . . . \\
\ldots . . .\end{array}$ & $\begin{array}{l}\mathrm{a} \\
\mathrm{b} \\
\mathrm{b} \\
\mathrm{c} \\
\mathrm{b}\end{array}$ \\
\hline $\begin{array}{l}561 \\
437\end{array}$ & & $\begin{array}{r}1,221 \\
738\end{array}$ & $\begin{array}{r}4.0 \\
220\end{array}$ & .2 & $88^{1.3}$ & & $\begin{array}{l}2,332 \\
2,041\end{array}$ & $\begin{array}{l}1,568 \\
1,405\end{array}$ & $\begin{array}{l}1,108 \\
1,047\end{array}$ & $\begin{array}{r}10 \\
7\end{array}$ & & & $\begin{array}{l}b \\
b\end{array}$ \\
\hline $\begin{array}{l}408 \\
321\end{array}$ & $\begin{array}{l}0 \\
0\end{array}$ & $\begin{array}{r}290 \\
8,470\end{array}$ & $\begin{array}{r}140 \\
24\end{array}$ & .2 & $\begin{array}{r}895 \\
11\end{array}$ & & $\begin{array}{r}2,430 \\
12,900\end{array}$ & $\begin{array}{l}1,430 \\
6,490\end{array}$ & $\begin{array}{l}1,100 \\
6,230\end{array}$ & $\begin{array}{r}6 \\
29\end{array}$ & $\begin{array}{r}2,860 \\
10,100\end{array}$ & $\begin{array}{l}8.0 \\
8.0\end{array}$ & a \\
\hline $\begin{array}{l}678 \\
305 \\
844\end{array}$ & $\begin{array}{l}33 \\
36 \\
35\end{array}$ & $\begin{array}{r}884 \\
1,110 \\
872\end{array}$ & $\begin{array}{r}18 \\
299 \\
58\end{array}$ & $\begin{array}{l}.2 \\
.4 \\
.6\end{array}$ & $\begin{array}{r}3.5 \\
1,180 \\
1.5\end{array}$ & $\begin{array}{l}.50 \\
.23 \\
.46\end{array}$ & $\begin{array}{l}2,020 \\
3,920 \\
2,220\end{array}$ & $\begin{array}{r}150 \\
2,320 \\
199\end{array}$ & $\begin{array}{r}0 \\
2,010 \\
0\end{array}$ & $\begin{array}{l}90 \\
18 \\
89\end{array}$ & $\begin{array}{l}2,960 \\
4,600 \\
3,330\end{array}$ & $\begin{array}{l}8.6 \\
8.5 \\
8.4\end{array}$ & $\begin{array}{l}a \\
a \\
a\end{array}$ \\
\hline $\begin{array}{l}566 \\
368 \\
396 \\
570 \\
490\end{array}$ & •....... & $\begin{array}{r}1,710 \\
754 \\
1,730 \\
1,840 \\
1,120\end{array}$ & $\begin{array}{r}34 \\
18 \\
24 \\
140 \\
85\end{array}$ & $\begin{array}{r}.6 \\
1.4 \\
.6 \\
.2 \\
.2\end{array}$ & $\begin{array}{c}1.6 \\
3.8 \\
14 \\
90 \\
6.5\end{array}$ & $\begin{array}{c}.40 \\
.46 \\
.42 \\
\ldots \ldots . .\end{array}$ & $\begin{array}{r}3,120 \\
1,520 \\
3,110 \\
\ldots \ldots \ldots \ldots . . .\end{array}$ & $\begin{array}{r}176 \\
672 \\
1,290 \\
2,300 \\
1,600\end{array}$ & $\begin{array}{r}0 \\
370 \\
965 \\
1,833 \\
1,198\end{array}$ & $\begin{array}{r}92 \\
40 \\
40 \\
\cdots . . \\
5\end{array}$ & $\begin{array}{r}4,070 \\
1,860 \\
3,010 \\
\ldots \ldots . . . .\end{array}$ & $\begin{array}{l}8.1 \\
6.8 \\
7.3 \\
\cdots . . .\end{array}$ & $\begin{array}{l}a \\
a \\
a \\
c \\
c\end{array}$ \\
\hline $\begin{array}{l}500 \\
580 \\
440 \\
412\end{array}$ & 12 & $\begin{array}{r}1,920 \\
630 \\
390 \\
1,880\end{array}$ & $\begin{array}{c}33 \\
310 \\
1.0 \\
19\end{array}$ & $\begin{array}{r}6.2 \\
.5 \\
.6\end{array}$ & $\begin{array}{r}170 \\
6.5 \\
.4\end{array}$ & .30 & $\begin{array}{l}3,280 \\
2,350 \\
1,120 \\
3,170\end{array}$ & $\begin{array}{r}2,350 \\
1,340 \\
700 \\
317\end{array}$ & $\begin{array}{r}1,940 \\
864 \\
339 \\
0\end{array}$ & \begin{tabular}{r|r}
3 \\
22 \\
12 \\
86
\end{tabular} & $\mid \begin{array}{r}\cdots \ldots \ldots \\
\cdots \ldots \ldots \\
\cdots, \ldots \ldots\end{array}$ & $\ldots \ldots$ & $\begin{array}{l}c \\
c \\
c \\
a\end{array}$ \\
\hline
\end{tabular}




\section{SALINE STREAMS AND LAKES}

Most of the streams in the Drift Prairie and Red River Valley are not saline. The major streams tributary to the Missouri River in the Missouri Plateau, however, are slightly saline at low flow. These tributary streams drain areas where rocks of Cretaceous and Tertiary age are exposed or lie close to the surface, and they are usually at low (base) flow except during periods of spring runoff and summer rains. The water during low-flow periods is predominantly of the sodium sulfate type.

Table 7. - Chemical analyses of

[Analytical results in parts per

\begin{tabular}{|c|c|c|c|c|c|c|c|c|c|c|}
\hline Date of collection & $\begin{array}{c}\text { Instan- } \\
\text { taneous } \\
\text { discharge } \\
(\mathrm{cfs})\end{array}$ & $\begin{array}{l}\text { Silica } \\
\left(\mathrm{SiO}_{2}\right)\end{array}$ & $\begin{array}{l}\text { Iron } \\
(\mathrm{Fe})\end{array}$ & $\begin{array}{c}\mathrm{Cal-} \\
\mathrm{Cium} \\
(\mathrm{Ca})\end{array}$ & $\begin{array}{c}\mathrm{Mag}- \\
\text { nesium } \\
(\mathrm{Mg})\end{array}$ & $\begin{array}{c}\text { Sodium } \\
(\mathrm{Na})\end{array}$ & $\begin{array}{c}\text { Potas- } \\
\text { sium } \\
(\mathrm{K})\end{array}$ & $\begin{array}{c}\mathrm{Bi}- \\
\mathrm{car}- \\
\text { bonate } \\
\left(\mathrm{HCO}_{3}\right)\end{array}$ & $\begin{array}{l}\mathrm{Car}- \\
\text { bon- } \\
\text { ate } \\
\left(\mathrm{CO}_{3}\right)\end{array}$ & $\begin{array}{l}\text { Sulfate } \\
\left(\mathrm{SO}_{4}\right)\end{array}$ \\
\hline
\end{tabular}

Des Lacs River

\begin{tabular}{|c|c|c|c|c|c|c|c|c|c|}
\hline $\begin{array}{l}\text { Apr. } 5,1949 \ldots \ldots . \\
\text { Jan. } 17,1950 \ldots \ldots \\
\text { July } 24,1950 \ldots \ldots .\end{array}$ & $\begin{array}{c}1,590 \\
30.4\end{array}$ & $\begin{array}{l}8.4 \\
26 \\
13\end{array}$ & $\begin{array}{c}0.15 \\
\cdots . . . \\
.02\end{array}$ & $\begin{array}{l}15 \\
71 \\
39\end{array}$ & $\begin{array}{l}3.0 \\
82 \\
26\end{array}$ & $\begin{array}{r}12 \\
325 \\
153^{\circ}\end{array}$ & $\begin{array}{r}67 \\
728 \\
354\end{array}$ & $\begin{array}{l}0 \\
0 \\
0\end{array}$ & $\begin{array}{r}17 \\
530 \\
210\end{array}$ \\
\hline
\end{tabular}

Little Missouri River

\begin{tabular}{|l|c|c|r|r|r|r|r|r|r|}
\hline July $23,1948 \ldots \ldots$ & 340 & 13 & 0.00 & 82 & 25 & 129 & 136 & 0 & 450 \\
Dec. 19, $1949 \ldots \ldots .$. & & .3 & .06 & 46 & 46 & 533 & 656 & 0 & 885 \\
Apr. $4,1950 \ldots \ldots$ & 2,730 & 8.0 & .16 & 15 & 3.5 & 19 & 68 & 0 & 34 \\
\hline
\end{tabular}

Little Missouri River

\begin{tabular}{|c|c|c|c|c|c|c|c|c|c|c|}
\hline $\begin{array}{l}\text { Jan. } 1 \text { to Feb. 4, } \\
1948 \ldots \ldots \ldots \ldots \ldots \ldots\end{array}$ & & 18 & 0.02 & 123 & 54 & 525 & 11 & 622 & 0 & 1,100 \\
\hline Mar. $19,1948 \ldots .$. & ${ }^{1} 5,120$ & 7.3 & .06 & 18 & 5.3 & 28 & & 100 & 0 & 37 \\
\hline $\begin{array}{l}\text { Mar. 11 to Sept. 30, } \\
1947^{2} \ldots \ldots \ldots \ldots \ldots \ldots . .\end{array}$ & 11,360 & 8.8 & .24 & 50 & 15 & 80 & 14 & 175 & 0 & 221 \\
\hline $\begin{aligned} \text { Oct. } 1,1947 \text { to } \\
\text { Sept. } 30,1948^{2} \text {. } \\
\text { Oct. } 1,1948 \text { to }\end{aligned}$ & 1457 & 14 & .07 & 44 & 13 & 97 & 3.3 & 163 & 0 & 235 \\
\hline Sept. $30,1949^{2}$. & ${ }^{1} 596$ & 12 & .02 & 32 & 14 & 50 & 2.5 & ${ }^{3} 136$ & .......... & 133 \\
\hline
\end{tabular}

Little Missouri River

\begin{tabular}{|l|r|r|r|r|r|r|r|r|r|r|r|}
\hline Mar. $26,1947 \ldots$. & 25,400 & 7.8 & 0.60 & 42 & 8.7 & 35 & 3.8 & 172 & 0 & 69 \\
June $8,1948 \ldots$. & 670 & 17 & .00 & 61 & 24 & 263 & 294 & 0 & 552 \\
Sept. $8,1949 \ldots$. & 13 & 16 & .02 & 86 & 40 & 350 & 456 & 0 & 725 \\
\hline
\end{tabular}

Knife River north-

\begin{tabular}{|l|l|l|l|l|l|l|l|l|l|l|}
\hline Aug. $18,1950 \ldots . .$. & $\ldots . . . . . . . .$. & 8.0 & 0.04 & 34 & 17 & 474 & 6.5 & 656 & 0 & 595 \\
\hline
\end{tabular}

See footnotes at end of table. 
Chemical analyses of water from saline streams are given in table 7. For some stations, analyses represent the quality of the water at high, near-average, and low flows. Weighted-average analyses are shown for those stations where samples were collected daily. The averages were computed by weighting each determined concentration with water discharge; they represent approximately the chemical character of the water if all the water passing a station during the period of the weighted average were impounded and mixed. Most of the analyses in table 7 were taken from U. S. Geological Survey Water-Supply Papers 1050, 1102, $1132,1162,1187$, and 1198 .

saline streams in North Dakota

million except as indicated]

\begin{tabular}{|c|c|c|c|c|c|c|c|c|c|c|c|}
\hline \multirow{3}{*}{$\begin{array}{l}\text { Chlo- } \\
\text { ride } \\
(\mathrm{Cl})\end{array}$} & \multirow{3}{*}{$\begin{array}{l}\text { Fluo- } \\
\text { ride } \\
\text { (F) }\end{array}$} & \multirow{3}{*}{$\begin{array}{l}\text { Nitrate } \\
\left(\mathrm{NO}_{3}\right)\end{array}$} & \multirow{3}{*}{$\begin{array}{l}\text { Boron } \\
\text { (B) }\end{array}$} & \multicolumn{3}{|c|}{ Dissolved solids } & \multirow{2}{*}{\multicolumn{2}{|c|}{$\begin{array}{l}\text { Hardness } \\
\text { as } \mathrm{CaCO}_{3}\end{array}$}} & \multirow{3}{*}{$\begin{array}{l}\text { Per- } \\
\text { cent } \\
\text { so- } \\
\text { dium }\end{array}$} & \multirow{3}{*}{$\begin{array}{l}\text { Specific } \\
\text { conduct- } \\
\text { ance } \\
\text { (micro- } \\
\text { mhos at } \\
\left.25^{\circ} \mathrm{C}\right)\end{array}$} & \multirow[b]{3}{*}{$\mathrm{pH}$} \\
\hline & & & & \multicolumn{2}{|c|}{ Parts per million } & \multirow[b]{2}{*}{$\begin{array}{l}\text { Tons } \\
\text { per } \\
\text { acre- } \\
\text { foot }\end{array}$} & & & & & \\
\hline & & & & $\begin{array}{l}\text { Residue } \\
\text { on } \\
\text { evapo- } \\
\text { ration } \\
\text { at } 180^{\circ} \mathrm{C}\end{array}$ & Sum & & $\begin{array}{l}\text { Cal- } \\
\text { cium, } \\
\text { mag- } \\
\text { nesium }\end{array}$ & $\begin{array}{c}\text { Non- } \\
\text { car- } \\
\text { bonate }\end{array}$ & & & \\
\hline
\end{tabular}

at Foxholm

\begin{tabular}{|c|c|r|c|r|r|r|r|r|r|r|r|r}
\hline 0.8 & 0.1 & 3.9 & 0.01 & 115 & $\ldots \ldots \ldots \ldots$ & 0.16 & 50 & & 0 & 35 & 157 & 7.2 \\
46 & $\ldots \ldots \ldots$ & 8.7 & $\ldots \ldots \ldots$ & 1,500 & 1,450 & 2.04 & 514 & & 0 & 58 & 2,000 & 7.8 \\
18 & .2 & 2.6 & $\ldots \ldots \ldots .$. & 682 & $\ldots \ldots \ldots \ldots$ & .93 & 205 & & 0 & 62 & 928 & 7.5 \\
\hline
\end{tabular}

at Marmarth

\begin{tabular}{|r|r|r|r|r|r|r|r|r|r|r|r}
\hline 4.0 & 0.2 & 2.6 & 0.21 & 842 & $\ldots \ldots \ldots .$. & 1.15 & 307 & 195 & 48 & 1,090 & 7.8 \\
2.0 & .6 & .9 & $\ldots \ldots \ldots$ & 1,910 & 1,850 & 2.60 & 304 & 0 & 79 & 2,570 & 8.1 \\
.0 & .2 & 1.9 & $\ldots \ldots \ldots$. & 138 & $\ldots \ldots \ldots \ldots$ & .19 & 52 & 0 & 44 & 187 & 6.7 \\
\hline
\end{tabular}

\section{at Medora}

\begin{tabular}{|c|c|c|c|c|c|c|c|c|c|c|c|}
\hline 15 & 0.4 & 1.0 & 0.27 & 2,200 & 2,160 & 2.99 & 529 & 19 & 68 & 2,800 & 8.2 \\
\hline 2.0 & .6 & 3.0 & ........... & 182 & ..m.n..... & .25 & 67 & 0 & 48 & 247 & 7.3 \\
\hline 2.6 & .3 & 1.7 & ........... & 484 & n............. & .66 & 186 & 42 & 46 & 704 & n.... \\
\hline 3.2 & .5 & 2.1 & .13 & 520 & ............... & .71 & 164 & 30 & 56 & 716 & $\ldots .$. \\
\hline .5 & .2 & 1.6 & .14 & 336 & n............ & .46 & 138 & 26 & 44 & 494 & $\ldots$. \\
\hline
\end{tabular}

near Watford City

\begin{tabular}{|r|r|r|r|r|r|r|r|r|r|r|r|r}
\hline 3.8 & 0.1 & 3.0 & 0.12 & 260 & $\ldots \ldots \ldots$ & 0.35 & 141 & 0 & 34 & 417 & 7.7 \\
4.1 & .3 & 1.7 & $\ldots \ldots \ldots$ & 1,110 & 1,070 & 1.51 & 251 & 10 & 70 & 1,420 & 7.7 \\
6.5 & .4 & 2.2 & .39 & 1,510 & 1,450 & 2.05 & 379 & 5 & 67 & 2,030 & 8.1 \\
\hline
\end{tabular}

west of Manning

\begin{tabular}{|c|c|c|c|c|c|c|c|c|c|c|c|}
\hline 4.7 & 0.6 & 2.7 & 0.10 & 1,500 & 1,460 & 2.04 & 154 & 0 & 86 & 2,150 & 7.9 \\
\hline
\end{tabular}


Table 7.- Chemical analyses of saline

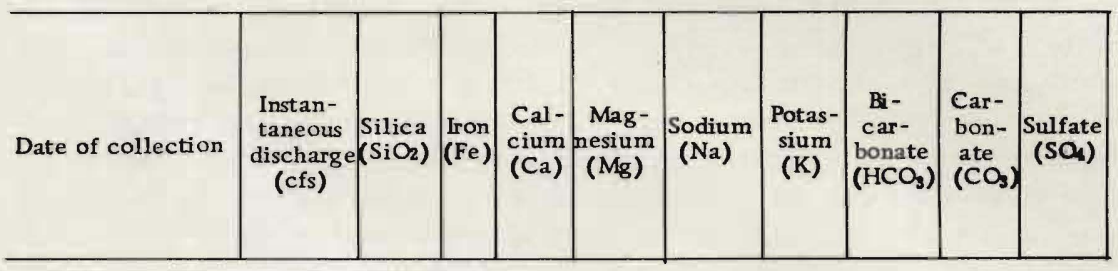

Crooked Creek

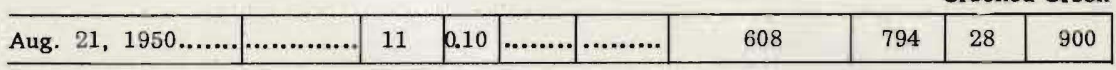

Knife River near

\begin{tabular}{|c|c|c|c|c|c|c|c|c|c|c|}
\hline $\begin{array}{l}\text { July } 19,1946 \ldots \ldots . \\
\text { Mar. } 25,1947 \ldots \ldots . \\
\text { Oct. } 19,1948 \ldots \ldots .\end{array}$ & $\begin{array}{c}123 \\
1,700 \\
6.3\end{array}$ & 23.6 & $\begin{array}{c}0.02 \\
\ldots \ldots \\
.11\end{array}$ & $\begin{array}{l}30 \\
10 \\
41\end{array}$ & $\begin{array}{l}13 \\
4.5 \\
35\end{array}$ & $\left.28\right|_{398} ^{83}$ & 7.6 & $\begin{array}{r}177 \\
90 \\
664\end{array}$ & $\begin{array}{r}10 \\
0 \\
20\end{array}$ & $\begin{array}{r}138 \\
18 \\
502\end{array}$ \\
\hline
\end{tabular}

\begin{tabular}{|c|c|c|c|c|c|c|c|c|c|c|}
\hline & & & & & & & & & Knif & River \\
\hline Aug. $23,1950$. & ….......... & 15 & 0.08 & 51 & 34 & 315 & 8.2 & 628 & 0 & 433 \\
\hline
\end{tabular}

Knife River

\begin{tabular}{l|c|c|c|c|c|c|c|r|r|r|}
\hline May & $31,1949 \ldots .$. & 181 & 10 & 0.01 & 50 & 33 & 356 & 632 & 0 & 488 \\
May & $18,1950 \ldots . .$. & 357 & 11 & .10 & 47 & 20 & 130 & 278 & 6 & 230 \\
Mar. $31,1951 \ldots . .$. & 7.600 & 4.1 & .40 & 14 & 2.2 & 20 & 66 & 0 & 27 \\
\hline
\end{tabular}

Heart River near

\begin{tabular}{|c|c|c|c|c|c|c|c|c|c|c|}
\hline Jan. $12-20,1948 \ldots$ & ${ }^{1} 0.6$ & 23 & 0.02 & 38 & 30 & 712 & 9.2 & 1,070 & 0 & 869 \\
\hline $\begin{array}{l}\text { Mar. } 31 \text { to Apr. 2, } \\
1949 . \ldots \ldots \ldots \ldots . . . . . .\end{array}$ & $\mathbf{l}_{2}, 060$ & 7.9 & .24 & 10 & 1.9 & 15 & 3.2 & 48 & 0 & 26 \\
\hline $\begin{array}{l}\text { May } 17 \text { to Sept. } 30, \\
1947^{2} \ldots \ldots \ldots \ldots . . . .\end{array}$ & 151 & 14 & .08 & 26 & 10 & 72 & 7.3 & ${ }^{3} 171$ & & 124 \\
\hline $\begin{array}{l}\text { Oct. } 1,1947 \text { to } \\
\text { Sept. } 30,1948^{2} \ldots \\
\text { Mar. } 1 \text { to Sept. } 27,\end{array}$ & $1_{31}$ & 9.8 & .23 & 14 & 5.0 & 46 & 11 & ${ }^{3} 107$ & & 73 \\
\hline $1949^{2} \ldots \ldots \ldots \ldots$ & 185 & 11 & .28 & 13 & 3.1 & 24 & 2.8 & 364 & ......... & 39 \\
\hline
\end{tabular}

\begin{tabular}{|l|l|l|l|l|l|l|l|l|l|}
\hline Aug. $19,1950 \ldots . .$. & $\ldots . . . \ldots . .$. & 16 & 0.24 & 49 & 33 & 322 & 504 & 0 & 515 \\
\hline
\end{tabular}

Cedar Creek above mouth of

\begin{tabular}{|l|l|l|l|l|l|l|l|l|l|}
\hline Aug. $29,1950 \ldots . .$. & $\ldots . . . . . . .$. & 8.0 & 0.06 & 102 & 61 & 270 & 436 & 0 & 688 \\
\hline
\end{tabular}

\begin{tabular}{|l|l|l|l|l|l|l|l|l|l|}
\hline Aug. 30, $1950 \ldots . .$. & $\ldots . . . . . . .$. & 11 & 0.04 & 109 & 97 & 356 & 356 & 0 & 1,090 \\
\hline
\end{tabular}

Cedar Creek

\begin{tabular}{|c|c|c|c|c|c|c|c|c|c|c|}
\hline $\begin{array}{l}\text { Mar. } 22,1947 \ldots \ldots . . \\
\text { Aug. } 15,1950 \ldots \ldots . . \\
\text { Dec. } 7,1950 \ldots \ldots .\end{array}$ & $\begin{array}{r}548 \\
148 \\
5.5\end{array}$ & $\begin{array}{l}3.6 \\
12 \\
15\end{array}$ & $\begin{array}{l}1.0 \\
.04 \\
.04\end{array}$ & $\begin{array}{l}14 \\
54 \\
67\end{array}$ & $\begin{array}{l}4.5 \\
31 \\
74\end{array}$ & $\begin{array}{r}111 \\
147 \\
366\end{array}$ & 7.3 & $\begin{array}{r}76 \\
224 \\
441\end{array}$ & $\begin{array}{l}0 \\
0 \\
0\end{array}$ & $\begin{array}{r}17 \\
368 \\
845\end{array}$ \\
\hline
\end{tabular}

See footnotes at end of table. 
streams in North Dakota-Continued

\begin{tabular}{|c|c|c|c|c|c|c|c|c|c|c|c|}
\hline \multirow{3}{*}{$\begin{array}{l}\text { Chlo- } \\
\text { ride } \\
(\mathrm{Cl})\end{array}$} & \multirow{3}{*}{$\begin{array}{l}\text { Fluo- } \\
\text { ride } \\
\text { (F) }\end{array}$} & \multirow[b]{3}{*}{$\begin{array}{r}\text { Nitrate } \\
\left(\mathrm{NO}_{3}\right)\end{array}$} & \multirow[b]{3}{*}{$\begin{array}{l}\text { Boron } \\
\text { (B) }\end{array}$} & \multicolumn{3}{|c|}{ Dissolved solids } & \multirow{2}{*}{\multicolumn{2}{|c|}{$\begin{array}{l}\text { Hardness } \\
\text { as } \mathrm{CaCO}_{3}\end{array}$}} & \multirow{3}{*}{$\begin{array}{r}\text { Per- } \\
\text { cent } \\
\text { so- } \\
\text { dium }\end{array}$} & \multirow{3}{*}{$\begin{array}{l}\text { Specific } \\
\text { conduct- } \\
\text { ance } \\
\text { (micro- } \\
\text { mhos at } \\
25^{\circ} \mathrm{C} \text { ) }\end{array}$} & \multirow[b]{3}{*}{$\mathrm{pH}$} \\
\hline & & & & \multicolumn{2}{|c|}{ Parts per million } & \multirow[b]{2}{*}{$\begin{array}{l}\text { Tons } \\
\text { per } \\
\text { acre- } \\
\text { foot }\end{array}$} & & & & & \\
\hline & & & & $\begin{array}{c}\text { Residue } \\
\text { on } \\
\text { evapo- } \\
\text { ration } \\
\text { at } 180^{\circ} \mathrm{C}\end{array}$ & Sum & & $\begin{array}{l}\text { Cal- } \\
\text { cium, } \\
\text { mag- } \\
\text { nesium }\end{array}$ & $\begin{array}{l}\text { Non- } \\
\text { car- } \\
\text { bonate }\end{array}$ & & & \\
\hline
\end{tabular}

at Emerson

\begin{tabular}{|l|l|l|l|l|l|l|l|l|l|l|l}
\hline 5.0 & 0.6 & 0.7 & $\ldots \ldots .$. & 2,110 & $\ldots \ldots \ldots$ & 2.87 & 321 & 0 & 80 & 2,830 & 8.3 \\
\hline
\end{tabular}

Golden Valley

\begin{tabular}{|c|r|r|r|r|r|r|r|r|r|r|r|}
\hline 0.9 & 0.4 & 1.4 & $\ldots \ldots .$. & 412 & $\ldots \ldots \ldots .$. & 0.56 & 128 & 0 & 58 & 543 & 8.2 \\
15 & .0 & .2 & 0.06 & 130 & $\ldots \ldots \ldots$ & .18 & 43 & 0 & 53 & 193 & 7.6 \\
6.0 & .6 & 1.0 & .14 & 1,400 & 1,360 & 2.16 & 246 & 0 & 78 & 1,870 & 8.2 \\
\hline
\end{tabular}

near Zap

\begin{tabular}{|l|l|l|l|l|l|l|l|l|l|l|l}
\hline 5.0 & 0.2 & 1.7 & 0.30 & 1,200 & 1,170 & 1.63 & 267 & 0 & 71 & 1,690 & 8.0 \\
\hline
\end{tabular}

at Hazen

\begin{tabular}{|r|r|r|r|r|r|r|r|r|r|r|r}
\hline 3.4 & 0.6 & 1.6 & $\ldots \ldots .$. & 1,350 & 1,260 & 1.84 & 261 & 0 & 75 & 1,870 & 7.9 \\
1.0 & .2 & 2.8 & $\ldots \ldots \ldots$ & 600 & $\ldots \ldots \ldots .$. & .82 & 200 & 0 & 59 & 899 & 8.2 \\
1.0 & .4 & 2.9 & 0.12 & 128 & $\ldots \ldots \ldots .$. & .17 & 44 & 0 & 49 & 170 & 7.2 \\
\hline
\end{tabular}

South Heart

\begin{tabular}{|c|c|c|c|c|c|c|c|c|c|c|c|}
\hline 7.0 & 0.7 & 13 & 1.1 & 2,270 & 2,230 & 3.09 & 218 & 0 & 87 & 3,100 & 8.2 \\
\hline 1.0 & .6 & 2.7 & .20 & 112 & ........... & .15 & 33 & 0 & 47 & 136 & 7.6 \\
\hline 1.3 & .1 & .6 & ........ & 356 & & .48 & 106 & 0 & 58 & 550 & $\ldots$ \\
\hline .8 & .3 & 1.8 & ......... & 233 & ............ & .32 & 56 & 0 & 59 & 314 & .... \\
\hline 4.7 & .5 & 2.0 & .13 & 148 & .......... & .20 & 45 & 0 & 52 & 198 & ....... \\
\hline
\end{tabular}

near New England

\begin{tabular}{|l|l|l|l|l|l|l|l|l|l|l|l}
\hline 5.0 & 0.4 & 0.2 & $\ldots \ldots .$. & 1,200 & 1,190 & 1.63 & 258 & 0 & 73 & 1,740 & 7.5 \\
\hline
\end{tabular}

Chantapeta Creek

\begin{tabular}{|l|l|l|l|l|l|l|l|l|l|l|l|}
\hline 10 & 0.6 & 5.0 & $\ldots \ldots .$. & 1,420 & 1,360 & 1.93 & 506 & 148 & 54 & 1,950 & 7.5 \\
\hline
\end{tabular}

above mouth

\begin{tabular}{|l|l|l|l|l|l|l|l|l|l|l|l}
\hline 11 & 0.4 & 3.3 & $\ldots \ldots .$. & 1,940 & 1,850 & 2.64 & 671 & 379 & 54 & 2,490 & 7.2 \\
\hline
\end{tabular}

near Pretty Rock

\begin{tabular}{|l|r|r|c|r|r|r|r|r|r|r|r}
\hline 4.0 & 0.0 & 0.2 & 0.04 & 116 & $\ldots \ldots \ldots$ & 0.16 & 53 & 0 & 27 & 169 & 7.4 \\
10 & .3 & 2.4 & $\cdots \ldots \ldots$ & 748 & $\cdots \ldots \ldots .$. & 1.02 & 263 & 79 & 55 & 1,080 & 7.5 \\
15 & .6 & 2.7 & .58 & 1,610 & 1,600 & 2.19 & 470 & 108 & 63 & 2,100 & 7.9 \\
\hline
\end{tabular}


Table 7.-Chemical analyses of saline

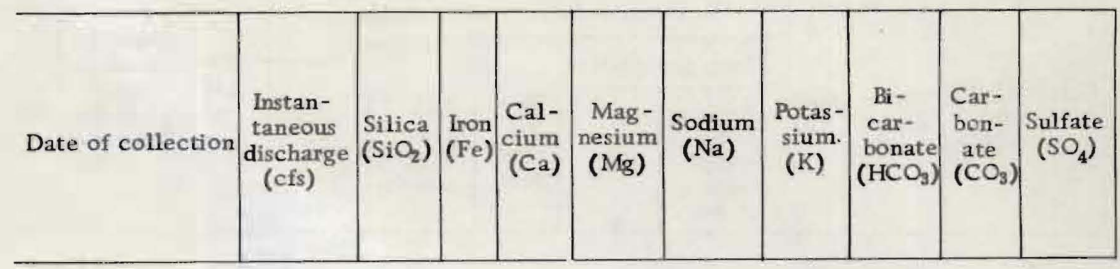

\begin{tabular}{|c|c|c|c|c|c|c|c|c|c|c|}
\hline & & & & & & & & \multicolumn{3}{|c|}{ North Fork Grand } \\
\hline Feb. $1-4,1952 \ldots$ & $\begin{array}{r}13.0 \\
1.70\end{array}$ & & ..... & & ........... & 924 & & 922 & 33 & 1,490 \\
\hline $\begin{array}{l}\text { Apr. } \\
\text { Feb. } 25-8 \text {-Sept. } 30,\end{array}$ & ${ }^{11} 11,080$ & & $\cdots$ & $\cdots \cdots \cdots$ & ............. & 13 & & 62 & 0 & \\
\hline $1951^{2} \ldots \ldots \ldots \ldots$ & ${ }^{1}{ }^{38.3}$ & 8.1 & 0.08 & 22 & 9.0 & 119 & 7.4 & ${ }^{3} 179$ & $\cdots$ & 198 \\
\hline $\begin{array}{l}\text { Oct. } 1,1951- \\
\text { Sept. } 30,1952^{2} . \text {. }\end{array}$ & ${ }^{1} 143$ & $\cdots$ & ...... & ........ & ........... & 32 & & 381 & …… & 49 \\
\hline
\end{tabular}

${ }^{1}$ Mean daily discharge.

${ }^{2}$ Weighted average.

Figure 7 shows salinity-duration curves for the Little Missouri, Heart, and North Fork Grand Rivers. During the periods of record the streams were slightly saline about three-fourths of the time at Medora, South Heart, and Haley.

Except for the high-flow part of the flow-duration curves (see following table), the curves for the period of chemical-quality

Summary data from flow-duration curves

\begin{tabular}{|c|c|c|c|c|c|c|c|}
\hline & \multirow[b]{2}{*}{ Station } & \multirow{2}{*}{$\begin{array}{c}\text { Drainage } \\
\text { area } \\
\text { (sq mile) }\end{array}$} & \multirow{2}{*}{ Period of record } & \multicolumn{4}{|c|}{$\begin{array}{c}\text { Percent of days flow } \\
\text { was less than - }\end{array}$} \\
\hline & & & & $\begin{array}{c}1 \\
\mathrm{cfs}\end{array}$ & $\begin{array}{l}10 \\
\text { cfs }\end{array}$ & $\begin{array}{l}100 \\
\mathrm{cfs}\end{array}$ & $\begin{array}{l}1,000 \\
\mathrm{cfs}\end{array}$ \\
\hline $\begin{array}{l}\text { Little } \\
\text { Heart } \\
\text { North }\end{array}$ & $\begin{array}{l}\text { Missouri River at Medora.. } \\
\text { River near South Heart..... } \\
\text { Fork Grand River at Haley. }\end{array}$ & $\begin{array}{r}6,190 \\
315 \\
509\end{array}$ & $\begin{array}{l}\text { October } 1945-\text { September } 1953 . . \\
\text { June } 1947-\text {-September } 1953 . . . . . \\
\text { October } 1945-\text { September } 1953 . .\end{array}$ & $\begin{array}{r}8 \\
55 \\
25\end{array}$ & $\begin{array}{l}22 \\
86 \\
80\end{array}$ & $\begin{array}{l}60 \\
96 \\
95\end{array}$ & $\begin{array}{l}90 \\
99 \\
99\end{array}$ \\
\hline
\end{tabular}

record closely approximate the curves for the period of streamflow record. The flow-duration data for the Heart and North Fork Grand Rivers are considered to be fairly representative of the upper reaches of the major streams tributary to the Missouri River and of the creeks and smaller streams in western North Dakota. The flow-duration data for the Little Missouri River at Medora are considered to be representative of the lower reaches of the major tributaries of the Missouri River in western North Dakota. 
streams in North Dakota-Continued

\begin{tabular}{|c|c|c|c|c|c|c|c|c|c|c|c|}
\hline \multirow[b]{3}{*}{$\begin{array}{l}\text { Chlo- } \\
\text { ride } \\
\text { (Cl) }\end{array}$} & \multirow[b]{3}{*}{$\begin{array}{l}\text { Fluo- } \\
\text { ride } \\
\text { (F) }\end{array}$} & \multirow[b]{3}{*}{$\begin{array}{r}\text { Nitrate } \\
\left(\mathrm{NO}_{3}\right)\end{array}$} & \multirow[b]{3}{*}{$\begin{array}{l}\text { Boron } \\
\text { (B) }\end{array}$} & \multicolumn{3}{|c|}{ Dissolved solids } & \multirow{2}{*}{\multicolumn{2}{|c|}{$\begin{array}{l}\text { Hardness } \\
\text { as } \mathrm{CaCO}_{3}\end{array}$}} & \multirow{3}{*}{$\begin{array}{l}\text { Per- } \\
\text { cent } \\
\text { so- } \\
\text { dium }\end{array}$} & \multirow{3}{*}{$\begin{array}{l}\text { Specific } \\
\text { conduct- } \\
\text { ance } \\
\text { (micro- } \\
\text { mhos at } \\
25^{\circ} \mathrm{C} \text { ) }\end{array}$} & \multirow[b]{3}{*}{$\mathrm{pH}$} \\
\hline & & & & Parts per & million & \multirow[b]{2}{*}{$\begin{array}{l}\text { Tons } \\
\text { per } \\
\text { acre- } \\
\text { foot }\end{array}$} & & & & & \\
\hline & & & & $\begin{array}{c}\text { Residue } \\
\text { on } \\
\text { evapo- } \\
\text { ration } \\
\text { at } 180^{\circ} \mathrm{C}\end{array}$ & Sum & & $\begin{array}{c}\text { Cal- } \\
\text { cium, } \\
\text { mag- } \\
\text { nesium }\end{array}$ & $\begin{array}{c}\text { Non- } \\
\text { car- } \\
\text { bonate }\end{array}$ & & & \\
\hline
\end{tabular}

River at Haley

\begin{tabular}{|c|c|c|c|c|c|c|c|c|c|c|c|}
\hline 12 & & & & 3,130 & $\ldots$. & 4.26 & 400 & 0 & 83 & 4,020 & 8.4 \\
\hline 1.3 & ......... & ............ & 0.28 & 112 & ............. & .15 & 47 & 0 & 38 & 149 & 7.3 \\
\hline 2.0 & 0.4 & 3.4 & .30 & 475 & & .65 & 92 & 0 & 72 & 698 & ... \\
\hline 1.3 & ... & …......... & .24 & 174 & & 2.37 & 56 & 0 & 58 & 247 & \\
\hline
\end{tabular}

${ }^{3}$ Includes carbonate as bicarbonate.

\section{SOURIS RIVER BASIN}

Chemical-quality data for several sites in the Souris River basin indicate that during periods of very low flow (less than about $5 \mathrm{cfs}$ ) the Des Lacs River is slightly saline at Foxholm. The water is of the sodium bicarbonate sulfate type; the maximum observed dissolved-solids concentration in samples taken at intervals of about a month during 1946-51 was 1,500 ppm in January 1950, and the minimum was 115 ppm in April 1949.

\section{LITTLE MISSOURI RIVER BASIN}

The chemical quality of the Little Missouri River was determined at Marmarth, at Medora, and near Watford City during 1945-51. Generally, the dissolved-solids concentration was greater than 1,000 ppm at Marmarth when the discharge was less than about $50 \mathrm{cfs}$ and at Medora and near Watford City when less than about $200 \mathrm{cfs}$.

The slightly saline water of the Little Missouri River is generally of the sodium sulfate type. The maximum observed concentration of dissolved solids was 2,230 ppm (discharge, 5.3 cfs) on January 2, 1951, at Marmarth. The weighted averages for the period October 1947 to September 1949 at Medora represent annual mean discharges similar to the average discharge, 539 cfs, for 9 years of record during 1923-51. 


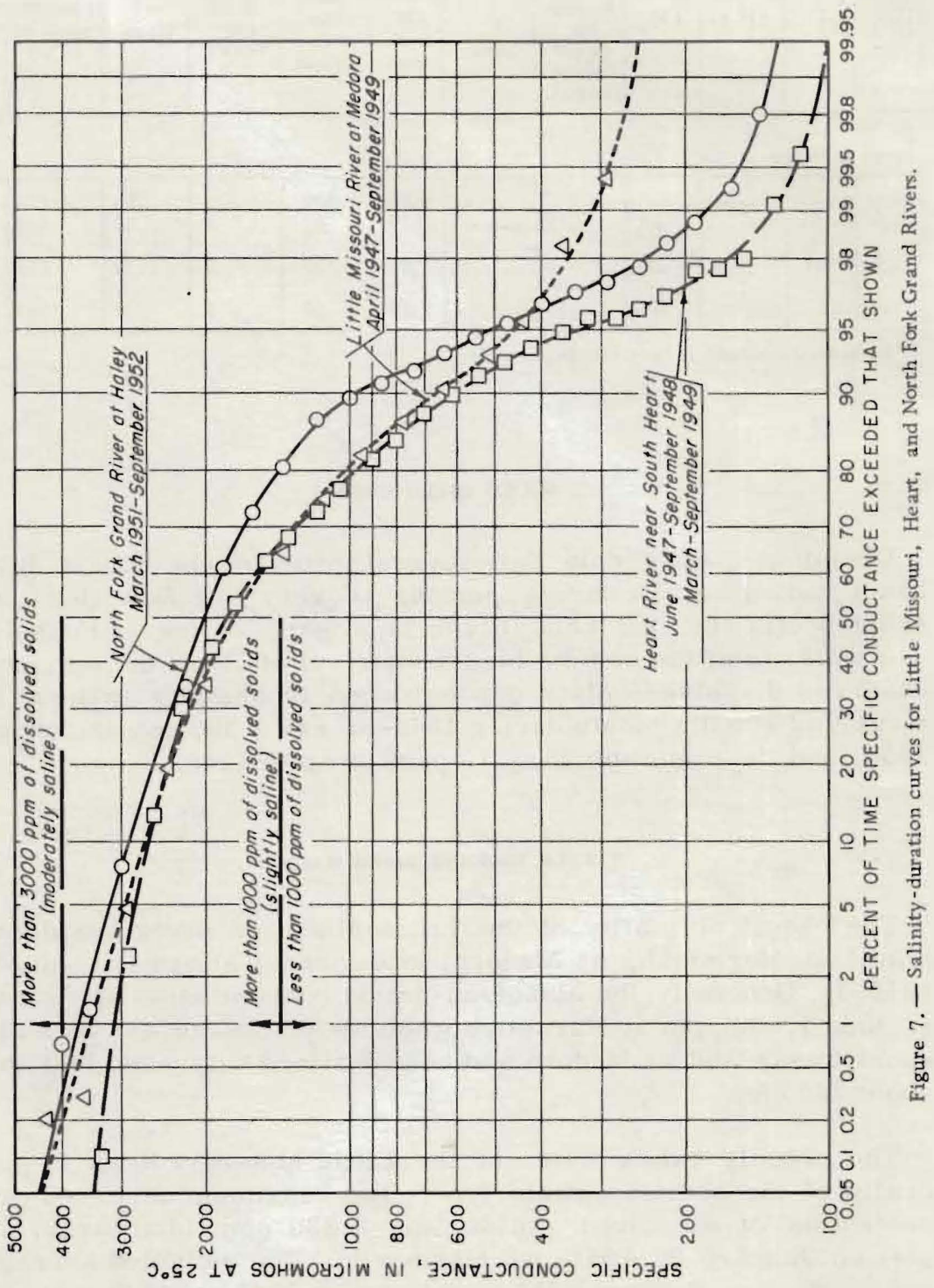


KNIFE RIVER BASIN

During the period 1945-51 the chemical quality of the Knife River near Golden Valley and at Hazen was investigated. Analyses of representative samples of high, near-average, and low flows at these sites are given in table 7 along with analyses of water from other sites on the Knife River and from Crooked Creek.

The maximum observed concentration of dissolved solids, which was in a sample from Crooked Creek, was 2,110 ppm. The waters of the basin are only slightly saline at low flow; sodium bicarbonate and sodium sulfate are the major salts in solution. Figure 8 shows the relation between specific conductance and discharge for the Knife River at Hazen. Dissolved solids in parts per million are approximately equal to 0.7 times the specific conductance in micromhos.

\section{HEART RIVER BASIN}

An unpublished report by Rainwater ${ }^{2}$ summarizes the results of studies of the chemical quality of water in the Heart River basin during 1945-50.

Table 7 shows the analyses of water from the Heart River near South Heart for low and high flows and the weighted averages for May 1947 to September 1949. Generally, the water is saline only at low flows (less than $10 \mathrm{cfs}$ near South Heart) and is of the sodium sulfate bicarbonate type. Table 8 shows maximum and minimum observed concentrations at several stationsalong the Heart River.

Analyses of samples collected from the Heart River below Heart Butte Dam near Glen Ullin in August and September 1950 indicated that releases from the reservoir were not saline. The dam was completed in time to impound the above-normal spring runoff of 1950 .

\section{CANNONBALL RIVER BASIN}

The chemical quality of the Cannonball River near New Leipzig and at Breien and of Cedar Creek near Pretty Rock was investigated during 1945-50. In addition, a salinity survey of the entire basin was made in August and September 1950. These investigations indicated that streams in the basin are slightly saline at

\footnotetext{
${ }^{2}$ Rainwater, F. H. , Chemical quality of surface waters in the Heart River basin, North Dakota: U. S. Geol. Survey Ms. rept.
} 


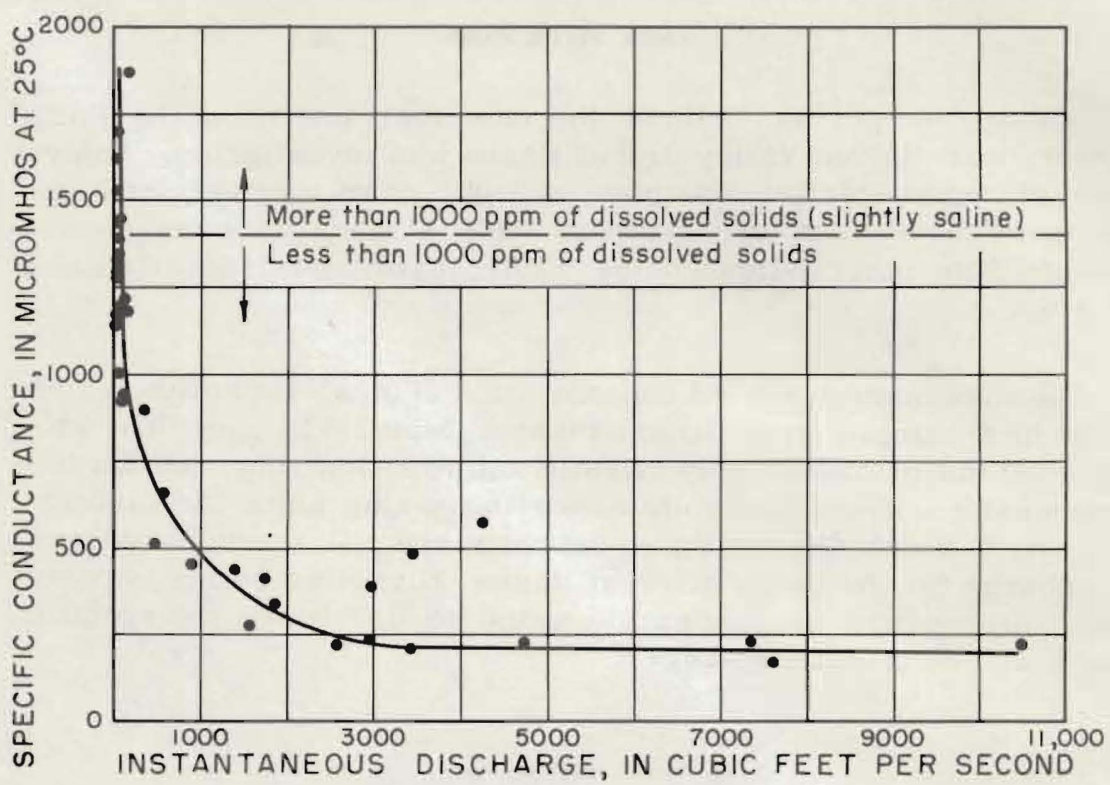

Figure 8. - Relation between water discharge and specific conductance, Knife River at Hazen, May 1946-September 1951.

low flow; the water is of the sodium sulfate bicarbonate type. Several analyses of saline surface waters in the basin are given in table 7 .

\section{GRAND RIVER BASIN}

The chemical quality of the North Fork Grand River at Haley near the South Dakota State line was studied during the period November 1950 to September 1952. During March 1951-September 1952 water in the North Fork was slightly saline about 80 percent of the time. (See fig. 7.) During spring runoff, dissolved-solids concentrations were as low as $112 \mathrm{ppm}$. However, concentrations of 2,000 and $3,000 \mathrm{ppm}$ were common during winter months; the maximum observed dissolved-solids concentration was $3,130 \mathrm{ppm}$ for February 1-4, 1952. Sodium sulfate and sodium bicarbonate are the major salts in solution. (See table 7.)

\section{DEVILS LAKE BASIN}

Some analyses of saline lakes in the Devils Lake basin are given in table 9 . The water in these lakes is of the sodium sulfate type, and the maximum observed concentration of dissolved solids was $116,000 \mathrm{ppm}$ (residue on evaporation) for a partial analysis of a sample collected in June 1949 from eastern Stump Lake. 


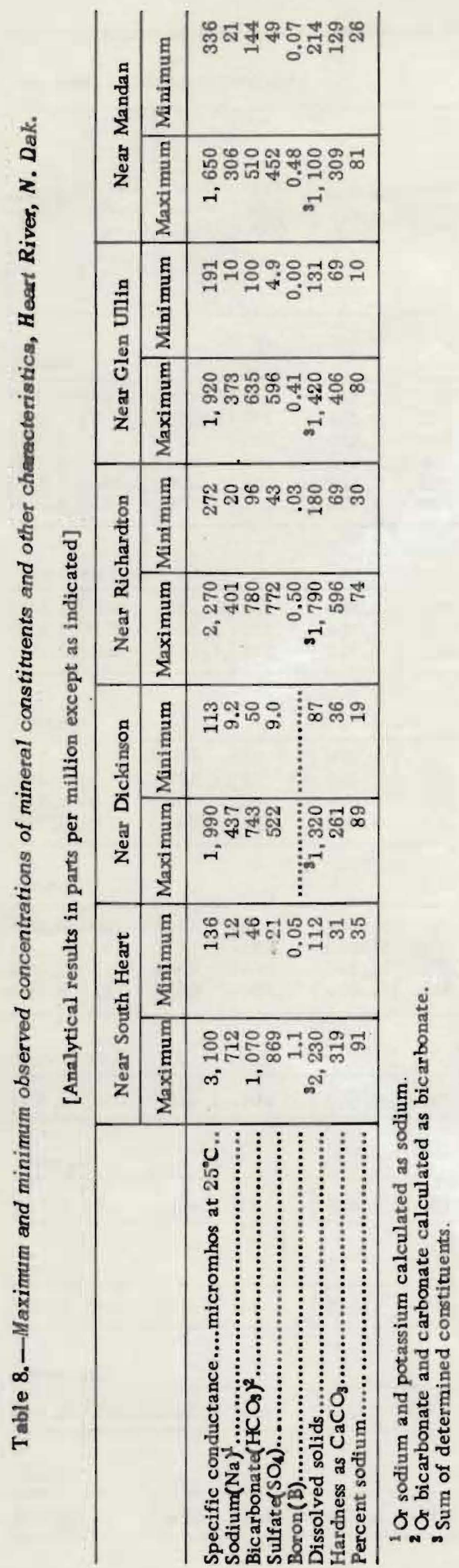


Table 9.-Chemical analyses of saline lakes in

[Analytical results in parts per

\begin{tabular}{|l|c|c|c|c|c|c|c|c|c|}
\hline Date of collection & $\begin{array}{c}\text { Silica } \\
\left(\mathrm{SiO}_{2}\right)\end{array}$ & $\begin{array}{l}\text { Iron } \\
(\mathrm{Fe})\end{array}$ & $\begin{array}{l}\mathrm{Ca1}- \\
\text { cium } \\
(\mathrm{Ca})\end{array}$ & $\begin{array}{c}\mathrm{Mag}- \\
\text { nesium } \\
(\mathrm{Mg})\end{array}$ & $\begin{array}{c}\text { Sodium } \\
(\mathrm{Na})\end{array}$ & $\begin{array}{c}\text { Potas- } \\
\text { sium } \\
(\mathrm{K})\end{array}$ & $\begin{array}{c}\text { Bicar- } \\
\text { bonate } \\
\left(\mathrm{HCO}_{3}\right)\end{array}$ & $\begin{array}{c}\text { Car- } \\
\text { bonate } \\
\left(\mathrm{CO}_{3}\right)\end{array}$ & $\begin{array}{c}\text { Sulfate } \\
\left(\mathrm{SO}_{4}\right)\end{array}$ \\
\hline
\end{tabular}

\begin{tabular}{|c|c|c|c|c|c|c|c|c|c|c|}
\hline & & & & & & & & & & Devils \\
\hline June & $14,1949 \ldots \ldots$ & & 0.20 & 79 & 605 & 2,870 & 242 & 708 & 60 & 6,380 \\
\hline July & $7,1950 \ldots \ldots$ & 9.5 & .04 & 54 & & & 177 & & 0 & 3,480 \\
\hline May & $22,1951 \ldots$ & 3.6 & & 90 & 286 & 1,650 & 160 & 583 & 0 & 3,450 \\
\hline May & $15,1952 \ldots$ & 7.6 & .16 & 39 & 350 & 1,760 & 175 & 524 & 75 & 3,700 \\
\hline July & $16,1954 \ldots \ldots \ldots \ldots \ldots$ & 11 & .04 & 120 & 398 & 2,250 & 236 & 666 & 81 & 4,780 \\
\hline June & $26,1955 \ldots \ldots \ldots \ldots . .$. & & & 81 & 316 & 1,730 & ...... & 558 & 75 & 3,650 \\
\hline
\end{tabular}

\begin{tabular}{l|c|c|c|c|c|r|r|r|r|r|}
\hline June $18,1949 \ldots \ldots \ldots \ldots . .$. & 9.2 & 0.09 & 26 & 1,870 & 10,300 & 832 & 1,120 & 365 & 23,500 \\
Aug. $1,1950 \ldots \ldots \ldots \ldots . .$. & 14 & .06 & 8.8 & 1,630 & 8,750 & 837 & 1,030 & 271 & 19,800 \\
May $14,1952 \ldots \ldots \ldots \ldots . .$. & 16 & .08 & 7.8 & 1,880 & 9,420 & 811 & 1,180 & 283 & 22,200 \\
\hline
\end{tabular}

\begin{tabular}{|c|c|c|c|c|c|c|c|c|c|c|}
\hline & & & & & & & & & \multicolumn{2}{|c|}{ Western } \\
\hline June & $16,1949 \ldots \ldots \ldots \ldots$ & 14 & 0.20 & 152 & 323 & 1,330 & 76 & 438 & $\begin{aligned} 0 \\
2\end{aligned}$ & \\
\hline $\begin{array}{l}\text { Aug. } \\
\text { May }\end{array}$ & 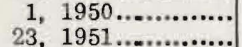 & $\begin{array}{l}9.1 \\
15\end{array}$ & \begin{tabular}{|l}
.08 \\
...$\cdots \ldots$ \\
\end{tabular} & $\begin{array}{r}96 \\
124\end{array}$ & $\begin{array}{l}104 \\
207\end{array}$ & $\begin{array}{l}680 \\
990\end{array}$ & $\begin{array}{l}70 \\
82\end{array}$ & $\begin{array}{l}288 \\
356\end{array}$ & $\begin{array}{r}22 \\
0\end{array}$ & $\begin{array}{l}1,400 \\
2,170\end{array}$ \\
\hline May & $13,1952 \ldots \ldots \ldots \ldots$ & 9.4 & .20 & 53 & 302 & 1,240 & 102 & 354 & 0 & 2,700 \\
\hline
\end{tabular}

\begin{tabular}{|c|c|c|c|c|c|c|c|c|c|c|}
\hline & & & & & & & & & & Eastern \\
\hline June & $16,1949 \ldots \ldots$ & 16 & & 33 & 5,710 & 21,900 & 956 & 930 & 220 & \\
\hline Aug. & 1. $1950 \ldots \ldots \ldots \ldots$ & 13 & .06 & 4.0 & 4,130 & 14,300 & 985 & 711 & 107 & 36,200 \\
\hline May & $23,1951 \ldots \ldots \ldots \ldots$ & 11 & & 133 & 3,970 & 14,100 & 860 & 797 & 77 & 35,900 \\
\hline May & $13,1952 \ldots \ldots \ldots \ldots$ & 12 & .08 & 7.6 & 4,480 & 15,000 & 1,000 & 849 & 121 & 39,500 \\
\hline
\end{tabular}

Free Peoples

\begin{tabular}{|l|l|l|l|l|l|l|l|l|l|}
\hline June $20,1949 \ldots \ldots . . . . .$. & 38 & 0.60 & 80 & 92 & 2,810 & 104 & 1,410 & 358 & 3,600 \\
\hline
\end{tabular}

Sixmile

\begin{tabular}{|l|l|l|l|l|l|l|r|r|r|}
\hline June $20,1949 \ldots \ldots \ldots . . .$. & 16 & 0.02 & 64 & 120 & 488 & 52 & 409 & 0 & 1,150 \\
\hline
\end{tabular}

\begin{tabular}{|c|c|c|c|c|c|c|c|c|c|c|}
\hline & & & & & & & & & & Spring \\
\hline June & $20,1949 \ldots \ldots \ldots \ldots$ & 13 & 0.06 & 112 & 280 & 819 & 55 & 406 & 24 & 2,300 \\
\hline
\end{tabular}

\begin{tabular}{|l|l|l|l|l|l|l|l|l|l|}
\hline June $17,1949 \ldots . . . . . . . .$. & 22 & 0.02 & 4.0 & 5.0 & 8,220 & 65 & 2,250 & 3,270 & 9,730 \\
\hline
\end{tabular}

\begin{tabular}{|l|l|l|l|l|l|l|l|l|l|}
\hline June $17,1949 \ldots \ldots . . . . .$. & 17 & 0.09 & 41 & 590 & 6,370 & 185 & 464 & 43 & 13,000 \\
\hline
\end{tabular}


the Devils Lake area in North Dakota

million except as indicated]

\begin{tabular}{|c|c|c|c|c|c|c|c|c|c|c|c|}
\hline \multirow{3}{*}{$\begin{array}{l}\text { Chlo- } \\
\text { ride } \\
\text { (Cl) }\end{array}$} & \multirow{3}{*}{$\begin{array}{l}\text { Fluo- } \\
\text { ride } \\
\text { (F) }\end{array}$} & \multirow{3}{*}{$\begin{array}{c}\text { Nitrate } \\
\left(\mathrm{NO}_{3}\right)\end{array}$} & \multirow{3}{*}{$\begin{array}{l}\text { Boron } \\
\text { (B) }\end{array}$} & \multicolumn{3}{|c|}{ Dissolved solids } & \multirow{2}{*}{\multicolumn{2}{|c|}{$\begin{array}{c}\text { Hardness } \\
\text { as } \\
\mathrm{CaCO}_{3}\end{array}$}} & \multirow{3}{*}{$\begin{array}{l}\text { Per- } \\
\text { cent } \\
\text { sodi- } \\
\text { um }\end{array}$} & \multirow{3}{*}{$\begin{array}{c}\text { Specific } \\
\text { conduct- } \\
\text { ance } \\
\text { (micro- } \\
\text { mhos at } \\
25^{\circ} \mathrm{C} \text { ) }\end{array}$} & \multirow[b]{3}{*}{$\mathrm{pH}$} \\
\hline & & & & \multicolumn{2}{|c|}{ Parts per million } & \multirow[b]{2}{*}{$\begin{array}{l}\text { Tons } \\
\text { per } \\
\text { acre- } \\
\text { foot }\end{array}$} & & & & & \\
\hline & & & & $\begin{array}{l}\text { Residue } \\
\text { on } \\
\text { evapo- } \\
\text { ration } \\
\text { at } 180^{\circ} \mathrm{C}\end{array}$ & Sum & & $\begin{array}{c}\text { Cal- } \\
\text { cium, } \\
\text { mag- } \\
\text { nesium }\end{array}$ & $\begin{array}{l}\text { Non- } \\
\text { car- } \\
\text { bon- } \\
\text { ate }\end{array}$ & & & \\
\hline
\end{tabular}

Lake

\begin{tabular}{|r|r|r|r|r|r|r|r|r|r|r|r}
\hline 1,330 & 2.0 & 19 & $\ldots \ldots \ldots$. & 12,500 & 12,500 & 17.0 & 2,680 & 2,000 & 68 & 13,800 & 8.4 \\
775 & .6 & 1.5 & $\ldots \ldots \ldots$. & 6,740 & 6,740 & 9.17 & 1,380 & 931 & 69 & 8,110 & 7.9 \\
743 & .2 & 5.2 & $\ldots \ldots \ldots$ & 6,700 & 6,680 & 9.11 & 1,400 & 922 & 69 & 8,340 & 8.1 \\
840 & .4 & 2.4 & .1 .1 & 7,390 & 7,210 & 10.1 & 1,540 & 986 & 69 & 9,140 & 8.8 \\
1,060 & 1.0 & .5 & 1.7 & 9,340 & 9,270 & 12.7 & 1,940 & 1,260 & 69 & 11,200 & 8.7 \\
788 & $\ldots \ldots .$. & $\ldots \ldots \ldots$. & $\ldots \ldots .$. & 7,080 & $\ldots \ldots \ldots .$. & 9.63 & 1,500 & 918 & 68 & 8,780 & 8.8 \\
\hline
\end{tabular}

Lake

\begin{tabular}{|r|r|r|r|r|r|r|r|r|r|r|r}
\hline 3,790 & 3.4 & 1.3 & $\ldots \ldots .$. & 45,100 & 41,300 & 61.3 & 7,750 & 6,220 & 72 & 38,300 & 8.7 \\
3,290 & 2.9 & 6.8 & $\ldots \ldots \ldots$ & 35,900 & 35,100 & 48.8 & 6,720 & 5,420 & 71 & 32,800 & 8.8 \\
3,590 & 2.9 & $\ldots \ldots \ldots$ & 4.8 & 39,900 & 38,800 & 54.3 & 7,750 & 6,310 & 70 & 37,000 & 8.6 \\
\hline
\end{tabular}

\section{Stump Lake}

\begin{tabular}{|r|r|r|r|r|r|r|r|r|r|r|r|}
\hline 875 & 1.0 & 5.9 & 1.9 & 6,470 & 6,100 & 8.80 & 1,710 & 1,350 & 62 & 7,790 & 8.0 \\
410 & 2.0 & .8 & $\ldots \ldots .$. & 3,110 & 2,940 & 4.23 & 667 & 394 & 66 & 4,080 & 8.4 \\
593 & .2 & 4.2 & $\ldots \ldots \ldots$ & 4,400 & 4,360 & 5.98 & 1,160 & 868 & 63 & 5,750 & 7.6 \\
750 & .4 & 3.1 & 1.1 & 5,520 & 5,340 & 7.51 & 1,380 & 1,090 & 64 & 7,150 & 8.1 \\
\hline
\end{tabular}

\section{Stump Lake}

\begin{tabular}{|r|c|c|c|r|r|r|r|r|r|r|r}
\hline 9,890 & 1.3 & 7.3 & 13 & 108,000 & 94,700 & 147 & 23,600 & 22,500 & 66 & 68,600 & 8.3 \\
7,390 & 2.8 & 10 & $\ldots \ldots .$. & 69,400 & 63,500 & 94.4 & 17,000 & 16,200 & 63 & 48,600 & 8.3 \\
7,140 & 2.7 & 37 & $\ldots \ldots \ldots$ & 66,000 & 62,600 & 89.8 & 16,700 & 15,900 & 63 & 52,800 & 8.3 \\
8,040 & 2.9 & $\ldots \ldots \ldots$ & 8.0 & 70,900 & 68,600 & 96.4 & 18,400 & 17,500 & 62 & 57,000 & 8.4 \\
\hline
\end{tabular}

Lake

\begin{tabular}{|r|r|r|r|r|r|r|r|r|r|r|r}
\hline 800 & 0.8 & 3.0 & $\ldots . .$. & 8,830 & 8,600 & 12.0 & 578 & 0 & 90 & 11,200 & 9.0 \\
\hline
\end{tabular}

Bay

\begin{tabular}{|r|r|r|r|r|r|r|r|r|r|r|r}
\hline 219 & 0.8 & 4.1 & $\ldots . . .$. & 2,480 & 2,320 & 3.37 & 653 & 318 & 60 & 3,370 & 7.9 \\
\hline
\end{tabular}

Lake

\begin{tabular}{|r|r|r|r|r|r|r|r|r|r|r|r}
\hline 380 & 0.4 & 1.3 & $\ldots \ldots .$. & 4,360 & 4,190 & 5.93 & 1,430 & 1,060 & 54 & 5,410 & 8.2 \\
\hline
\end{tabular}

Lake

\begin{tabular}{|c|c|c|c|c|c|c|c|c|c|c|c|}
\hline 591 & ........ & 3.8 & ........ & 23,500 & 23,100 & 32.0 & 31 & 0 & 99 & 25,600 & 9.8 \\
\hline
\end{tabular}

Lake

\begin{tabular}{|l|r|r|r|r|r|r|r|r|r|r|r}
\hline 1,670 & 3.4 & 2.1 & $\ldots . .$. & 23,300 & 22,200 & 31.7 & 2,530 & 2,080 & 83 & 23,900 & 8.3 \\
\hline
\end{tabular}


Table 9.-Chemical analyses of saline lakes in

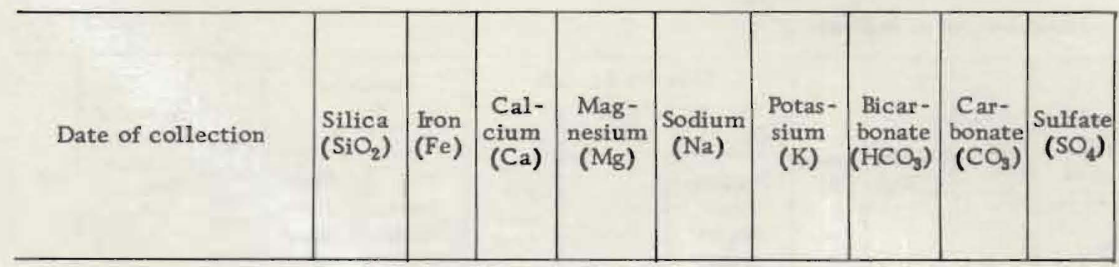

\begin{tabular}{|l|r|r|r|r|r|r|r|r|r|}
\hline June $20,1949 \ldots \ldots . . . . . .$. & 13 & 0.11 & 26 & 421 & 2,290 & 228 & 261 & 70 & 4,990 \\
\hline
\end{tabular}

\begin{tabular}{|l|l|l|l|l|l|l|l|l|l|}
\hline June $20,1949 \ldots \ldots . . . . . .$. & 49 & 0.40 & 120 & 708 & 3,470 & 216 & 708 & 73 & 7,360 \\
\hline
\end{tabular}

According to Swenson and Colby (1955), the salinity of these lakes is caused mainly by evaporation; surface runoff has been insufficient for many years to cause the lakes to overflow. The lakes lie in the valley of a preglacial river, which, with its tributaries, is partly filled with glacial drift (Upham, 1895, p. 170171). Surface inflow to Devils Lake is mostly from Mauvais Coulee, which is the largest stream in the basin and is intermittent. The lakes may also receive ground-water inflow from the lower part of the glacial drift, which is underlain by the Pierre shale. Evaporation from a lake surface in the vicinity of Devils Lake has been estimated at about 30 inches per year (Swenson and Colby, 1955), almost twice the mean annual precipitation.

Waters are classed as briny in eastern Stump and East Devils Lakes, very saline in Cranberry and Stink Lakes, and moderately saline in Devils, western Stump, Free Peoples, and Spring Lakes.

\section{SUMMARY}

Aquifers of Paleozoic age yield as much as 400 gpm of modererately saline to briny water of the sodium chloride type to wells in eastern North Dakota.

The Dakota sandstone, Fox Hills sandstone, and Hell Creek formation of Cretaceous age and the Fort Union formation of Tertiary age yield very small to large amounts of slightly saline to very saline water. 
the Devils Lake area in North Dakota-Continued

\begin{tabular}{|c|c|c|c|c|c|c|c|c|c|c|c|}
\hline \multirow{3}{*}{$\begin{array}{l}\text { Chlo- } \\
\text { ride } \\
\text { (Cl) }\end{array}$} & \multirow{3}{*}{$\begin{array}{l}\text { Fluo- } \\
\text { ride } \\
\text { (F) }\end{array}$} & \multirow{3}{*}{$\begin{array}{r}\text { Nitrate } \\
\left(\mathrm{NO}_{3}\right)\end{array}$} & \multirow{3}{*}{$\begin{array}{l}\text { Boron } \\
\text { (B) }\end{array}$} & \multicolumn{3}{|c|}{ Dissolved solids } & \multirow{2}{*}{\multicolumn{2}{|c|}{$\begin{array}{c}\text { Hardness } \\
\text { as } \\
\mathrm{CaCO}_{3}\end{array}$}} & \multirow{3}{*}{$\begin{array}{l}\text { Per- } \\
\text { cent } \\
\text { sodi- } \\
\text { um }\end{array}$} & \multirow{3}{*}{$\begin{array}{c}\text { Specific } \\
\text { conduct- } \\
\text { ance } \\
\text { (micro- } \\
\text { mhos at } \\
25^{\circ} \mathrm{C} \text { ) }\end{array}$} & \multirow[b]{3}{*}{$\mathrm{pH}$} \\
\hline & & & & \multicolumn{2}{|c|}{ Parts per million } & \multirow[b]{2}{*}{$\begin{array}{l}\text { Tons } \\
\text { per } \\
\text { acre- } \\
\text { foot }\end{array}$} & & & & & \\
\hline & & & & $\begin{array}{l}\text { Residue } \\
\text { on } \\
\text { evapo- } \\
\text { ration } \\
\text { at } 180^{\circ} \mathrm{C}\end{array}$ & Sum & & $\begin{array}{l}\text { Cal- } \\
\text { cium, } \\
\text { mag- } \\
\text { nesium }\end{array}$ & $\begin{array}{l}\text { Non- } \\
\text { car- } \\
\text { bon- } \\
\text { ate }\end{array}$ & & & \\
\hline
\end{tabular}

Bay

\begin{tabular}{|l|l|l|l|l|l|l|l|l|l|l|l|}
\hline 1,170 & 1.0 & 0.9 & 2.8 & 10,000 & 9,340 & 13.6 & 1,800 & 1,470 & 70 & 11,600 & 8.9 \\
\hline
\end{tabular}

Bay

\begin{tabular}{|l|r|r|r|r|r|r|r|r|r|r|r}
\hline 1,600 & 1.4 & 3.0 & $\ldots \ldots \ldots$ & 14,700 & 14,000 & 20.0 & 3,210 & 2,510 & 68 & 16,200 & 8.4 \\
\hline
\end{tabular}

Saline water from the Dakota sandstone is only slightly saline in the southeastern part of the State but is moderately saline farther north. Sodium chloride and sodium sulfate are the principal dissolved salts in the water.

Fresh water is obtained from the Fox Hills sandstone in the southwestern part of the State, and small supplies of slightly saline water of the sodium bicarbonate type are obtained farther east.

The Hell Creek and Fort Union formations crop out in a major part of the Missouri Plateau. Generally, saline water from the Hell Creek formation and upper part of the Fort Union formation is of the sodium bicarbonate sulfate type, and that from the Cannonball member of the Fort Union formation is of the sodium chloride type. Wells in these aquifers produce small to moderate supplies of slightly saline water in the western part of the State.

Glacial drift and alluvium of Quaternary age yield slightly saline water in widely varying amounts throughout much of the State. Because the hydrologic and lithologic characteristics of the drift and alluvial deposits are highly variable, the quantity and quality of the water from these deposits also are variable.

The major streams tributary to the Missouri River in western North Dakota are slightly saline at low flow. Sodium bicarbonate and sodium sulfate are the principal dissolved salts. Some lakes in the Devils Lake basin are briny; and others are very saline to slightly saline. 


\section{SELECTED BIBLIOGRAPHY}

Abbott, G. A., and Voedisch, F. W., 1938, The municipal ground-water supplies of North Dakota: N. Dak. Geol. Survey Bull. 11.

Akin, P. D., 1952, Ground water in the Litchville area, Barnes County, N. Dak.: N. Dak. State Water Conserv. Comm., and N. Dak. State Geol. Survey, N. Dak. Ground-Water Studies, no. 18.

Aronow, Saul, Dennis, P. E., and Akin, P. D., 1953, Geology and ground-water resources of the Michigan City area, Nelson County, N. Dak.: N. Dak. State Wat er Conserv. Comm., and N. Dak. StateGeol. Survey, N. Dak. Ground-WaterStudies, no. 21.

Dennis, P. E., 1947a, Ground water in the Aneta area, Nelson County, N. Dak.: N. Dak. State Water Conserv. Comm., and N. Dak. State Geol. Survey, N. Dak. Ground-Water Studies, no. 7.

1947b, Ground water near Buxton, Traill County, N. Dak,: N. Dak. State Water Conserv, Comm., and N. Dak. State Geol. Survey, N. Dak. Ground-Water Studies, no. 5. 1947c, Ground water in the Sharon area, Steele County, N. Dak.: N. Dak. State Water Conserv. Comm., and N. Dak. State Geol. Survey, N. Dak. Ground-Water Studies, no. 8.

1948a, Ground water in the Wimbledon area, Bames and Stutsman Counties, N. Dak.: N. Dak. State Water Conserv. Comm., and N. Dak. State Geol. Survey, N. Bak. Ground-Water Studies, no. 10.

-1948 b, Ground water in the Hope area, Steele County, N. Dak.: N. Dak. State Water Conserv. Comm., and N. Dak. State Geol. Survey, N. Dak. Ground-Water Studies, no. 9.

Dennis, P. E., and Akin, P. D., 1950, Ground water in the Portland area, Traill County, N. Dak.: N. Dak. State Water Conserv. Comm., and N. Dak. State Geol. Survey, N. Dak. Ground-Water Studies, no. 15.

Dingman, R. J., and Gordon, E. D., 1955, Geology and ground-water resources of the Fort Berthold Indian Reservation, N. Dak. U. S. Geol Survey Water-Supply Paper 1259.

Filaseta, Leonard, 1946, Ground water in the Fessenden area, Wells County, N. Dak.: N. Dak. State Water Conserv. Comm., and N. Dak. Geol. Survey, no. 1.

Kunkel, R. P., 1954, Structure contour map of the base of Mississippian rocks in the Williston Basin and adjoining areas of Montana, North Dakota, South Dakota, and Wyoming: U. S. Geol. Survey Oil and Gas Inv. Map OM 165.

Laird, W. M., and Towse, D. E., 1953, Stratigraphy of North Dakota with reference to oil possibilities: N. Dak. Geol. Survey Rept. Inv. 2.

North Dakota Geological Survey Circulars, 1949-55, Mimeographed logs of oil and gas test holes and wells.

Simpson, H. E., 1929, Geology and ground-water resources of North Dakota: U. S. Geol. Survey Water-Supply Paper 598.

Stiff, H. A., Jr., 1951, The interpretation of chemical water analysis by means of patterns: Petrol eum Tech. Jour., Tech. Note 84, sec. 1, p. 15-16.

Swenson, H. A., and Colby, B. R., 1955, Chemical quality of surface waters in the Devils Lake basin, North Dakota: U. S. Geol. Survey Water-Supply Paper 1295.

Tychsen, P. C., 1950, Geology and ground-water hydrology of the Heart River irrigation project and the Dickinson area, North Dakota, U. S. Geol. Survey Circ. 34.

Upham, Warren, 1895, The glacial Lake Agassiz: U. S. Geol. Survey Mon. 25.

U. S. Congress, 82d, 1952, An act to provide for research into ***water suitable for agricultural, industrial, municipal, and other beneficial consumptive uses, and for other purposes: 2d sess., Public Law 448. 


\section{INDEX}

Abstract,..........................................

Adams County........................................ 24

Altamont moraine ................................... 9

Aquifers, Cenozoic............................. 11, 15

interconnection

Mesozoic................................... 11, 15

Paleozoic..................................... 11, 15

saline water yielding...................... 9-11, $15-17,24-25,38$

Bibliography.

Bowman County

Brine, uses............................................ 15

Burleigh County.............................. 15, 24

\section{Cannonball member, saline} water, quality.

saline water, yleld:.......................... 11

Cannonball River basin....................... 63-64

Cavalier County.

Chemical analyses, saline ground water, Dakota sandstone........... 18-23

saline ground water, Fort Union and Hell Creek formations.

Fox Hills sandstone.0.0.0.

glacial drift and alluvium............. 40-55

Paleonoic formations.................... 16-17

saline lakes................................. 66-69

saline streams............................. 56-61

Chemical characteristics, saline water, consolidated rocks............... 12

saline water, glacial drift and alluvium... 13

Connate water.

Dakota sandstone, flowing wells.

saline water, analyses. quality. yield. stratigraphy.

etermined

Devils Lake area, saline lake analyses................ 66-69

Devils Lake basin.................................. 64

Dissolved solids concentration............... 2-3.

Drift Prairie. $8-9,24$

Equivalent per million, conversion factors

Flow-duration data.

Fort Union formation, saline water, analyses.

60

saline water, quality

$26-37$

38

tratigraphy
Fox Hills sandstone, saline

water, quality

Page

saline water, yield........................ 11

Fresh water, classification.................. 2-3

Fuson shale ........... 16

General geology............................. 8-9

Glacial drift and alluvium, saline water, analyses................ 40-55

saline water, quality................... 13, 38

yield.................................... 11, 38

Grand Forks County...................... 8, 15

Grand River basin................................. 64

Ground water, salinity processes......... 4, 7

Heart River, mineral constituents........... 65

Heart River basin.................................. 63

Hell Creek formation, saline water, analyses........... 26-37

saline water, quality...................... 38

yield........................................... 11

stratigraphy............................. 25

Introduction................................ 1

James River valley............................ 8

Kidder County............................. 24, 25

Knife River, water discharge and specific conductance................... 64

Knife River basin................................ 63

Lake Agassiz, glacial lake.................. 8

Lake Dakota, glacial lake................... 8

Lake Souris, glacial lake............. 8

Lakes, saline................................. 56-57

Lakot a sandstone.............................. 16

Little Missouri River basin................... 61

McIntosh County....................... 24, 25

McKenzie County ............................ 9

Map, geologic systems....................... 14

physiographic regions.................... pl. 1

saline-water analyses,

distribution................................. 6

Missouri Plateau.............................. 8,9 , degree of salinity............. 56, 60-65

Natural waters, definition.............. 1, 2-3

Paleonoic formation, saline

water, analyses.................... 16-17

saline water, quality....................... 15

yield 
Page

Pembina County ..........................11, 15

Percent sodium, definition .....................4

Physiographic regions $\ldots \ldots \ldots \ldots \ldots \ldots \ldots \ldots \ldots, 8$

Pierre shale ............................. 9, 24

Red River Valley, geology.............. 8, 11

Residue on evaporation.

Saline ground water, analyses,

Dakota sandstone ....................18-23

analyses, Fort Union and $\mathrm{Hell}$

Creek formations

26-37

Fox Hills sandstone....................24-25

glacial drift and alluvium ............. 40-55

Paleozoic formations ...................16-17

availability ............................. 1,2

Saline lakes, analyses ..................6. 66-69

Saline streams, analyses.................. 56-61

Saline water, classification.

$56-61$

occurrence .................. 2, 4, 7-8

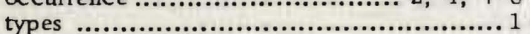

Saline Water Conversion Page

Program ............................. 1-2

Salinity-duration curves ................... 62

Scope of report ............................. 2

Sioux County .................................. 24

Souris River basin ..........................61 61

Specific conductance, definition ........... 3

Stratigraphic section,

saline-water aquifers ................ 10

Streams, saline ........................ 56-57

Summary .................................... 68

Surface water, salinity processes...........8

Turtle Mountains,$\ldots \ldots \ldots \ldots \ldots \ldots \ldots \ldots \ldots \ldots \ldots \ldots$

Walsh County .........................11, 15

Water, chemical changes................... 7

Well-numbering system................... 5

Williston Basin ..................... 8, 9, 11

Yields of aquifers, classification ............9 
